\title{
AN EVALUATION OF OPTICAL SWITCH ARCHITECTURES UTILIZING \\ WAVELENGTH CONVERTERS
}

\section{UNE ÉVALUATION DES ARCHITECTURES DE COMMUTATEUR OPTIQUE UTILISANT DES CONVERTISSEURS DE LONGUEUR D'ONDE}

\author{
A Thesis submitted \\ to the Department of Electrical and Computer Engineering \\ Royal Military College of Canada \\ by \\ Nicholas P. Torrington-Smith \\ Captain \\ In partial fulfillment of the requirements for the degree \\ Master of Engineering in Computer Engineering
}

April 2000

(C) Copyright by Capt N.P. Torrington-Smith 2000

This thesis may be used within the Department of National Defence but copyright for open publication remains the property of the author. 
National Library of Canada

Acquisitions and Bibliographic Services

395 Wellington Street Otlawa ON K1A ON4 Canada
Bibliothèque nationale du Canada

Acquisitions et services bibliographiques

395. rue Wellington Ottawa ON K1A ON4 Canada
The author has granted a nonexclusive licence allowing the National Library of Canada to reproduce, loan, distribute or sell copies of this thesis in microform, paper or electronic formats.

The author retains ownership of the copyright in this thesis. Neither the thesis nor substantial extracts from it may be printed or otherwise reproduced without the author's permission.
L'auteur a accordé une licence non exclusive permettant à la Bibliothèque nationale du Canada de reproduire, prêter, distribuer ou vendre des copies de cette thèse sous la forme de microfiche/film, de reproduction sur papier ou sur format électronique.

L'auteur conserve la propriété du droit d'auteur qui protège cette thèse. $\mathrm{Ni}$ la thèse ni des extraits substantiels de celle-ci ne doivent être imprimés ou autrement reproduits sans son autorisation. 
To my family with love. 


\section{ACKNOWLEDGEMENTS}

I would like to express my thanks to my thesis advisors, Dr. H.T. Mouftah and Dr. M. H. Rahman for their guidance and advice in helping me through the preparation of this thesis. I would also like to acknowledge Dr. Scott Knight for his help and insight with my $\mathrm{C}++$ programming exploits, and Dave DePlanché for his silent motivation and support.

I would also like to acknowiecige the help of Mr. John Campbell from Astarté Fibre Networks and Mr. John Nix from ADC/AOFR for their help with specific optical components used in my research.

The person who deserves most of my gratitude is my wife Brenda. Her love, support and understanding have been endless, and her continued motivation was a driving force to keep me going. I would also like to thank my son Ryan, whose smile brought me comfort during the days I needed it most. 


\begin{abstract}
Torrington-Smith, Nicholas P. M.Eng. (Comp. Eng.). Royal Military College of Canada. May 2000. An Evaluation Of Optical Switch Architectures Utilizing Wavelength Converters.

Supervisors: Dr. Hussein T. Mouftah, Dr. M. Hafizur Rahman.

All-optical networks utilizing wavelength division multiplexing (WDM) seems to be the way to the future. The bandwidth available in this type of network is more than sufficient to satisfy the projected bandwidth demands. However, due to the current costs of the network components, ways are being sought to maximize the benefits of optical networks while minimizing the costs.

Wavelength conversion has been studied as a way to improve efficiency in alloptical networks. A network utilizing wavelength conversion can minimize the number of wavelengths it employs, and thus reducing complexity and cost. However, the best placement of wavelength converters within the network still remains an unresolved issue.

This thesis looks at the four known switch architectures employing wavelength converters: dedicated, share-per-node, share-per-link, and electronic wavelength convertible switches. It also proposes a novel compromise between cost and performance with the half-clear wavelength convertible switch.

The half-clear wavelength convertible switch utilizes a bank of wavelength converters similar to that in the share-per-node architecture. However, the difference being only half of the wavelengths can be routed to the converter bank for clear conversion at any given node in the network.

Each of the switch architectures is evaluated by comparing the cost and attenuation at the switch components with emphasis being placed on the cost issues. The half-clear wavelength convertible switch is then be evaluated through simulation and compared with no wavelength conversion and the dedicated wavelength convertible switch architecture. It is shown that the half-clear wavelength convertible switch performs similar to the dedicated wavelength convertible switch, with a cost closer to opto-electronic techniques.
\end{abstract}

\title{
Kevwords:
}

Wavelength Conversion, Wavelength Division Multiplexing, Optical Networks 


\section{RÉSUMÉ}

Torrington-Smith, Nicholas P. M.Eng. (Comp. Eng.) Collège Militaire Royal du Canada. Mai 2000. Une Évaluation Des Architectures De Commutateur Optique Utilisant Des Convertisseurs De Longueur D'onde.

Directeurs de thèse : Dr Hussein T. Mouftah, Dr M. Hafizur Rahman.

Les réseaux tout-optique utilisant le multiplexage par répartition en longueur d'onde semble être la voie du futur. La largeur de bande disponible dans ce type de réseau est plus que suffisante pour satisfaire aux demandes de largeur de bande projetées. Cependant, en raison des coûts actuels des composants de réseaux, des voies sont recherchées afin de maximiser les avantages des réseaux optiques tout en réduisant au minimum les coûts.

La conversion de longueur d'onde a été étudiée afin d'améliorer l'efficacité dans les réseaux tout-optique. Un réseau utilisant la conversion de longueur d'onde peut réduire au minimum le nombre de longueurs d'onde qu'il utilise, réduisant de ce fait la complexité et le coût. Cependant, le meilleur emplacement des convertisseurs de longueur d'onde dans le réseau demeure toujours sans solution.

Cette thèse étudie les quatre architectures connues de commutateur utilisant des convertisseurs de longueur d'onde : dédié, part-par-noeud, part-par-lien et convertible électronique de longueur d'onde commute. Elle propose également un nouveau compromis entre le coût et la performance avec le commutateur convertible moitié-clair de longueur d'onde.

Le commutateur convertible de longueur d'onde moitié-clair utilise une banque de convertisseurs de longueur d'onde semblable à celui dans l'architecture de part-parnoeud. Cependant, la différence étant seulement la moitié des longueurs d'onde elle peut être conduite à la banque de convertisseur pour la conversion claire à n'importe quel noeud donné dans le réseau.

Chacune des architectures de commutateur est évaluée en comparaison de coút et d'atténuation aux composants de commutateur, l'accent étant mis sur les questions de coût. Le commutateur convertible à longueur d'onde moitié-claire commutateur convertible est ainsi évalué par la simulation et est alors comparé avec la longueur d'onde sans conversion et l'architecture convertible de commutateur de longueur d'onde dédiée. Il est démontré que le commutateur convertible de longueur d'onde moitié-claire performe de façon similaire au commutateur convertible de longueur d'onde dédiée avec un coût plus près des techniques opto-électroniques. 


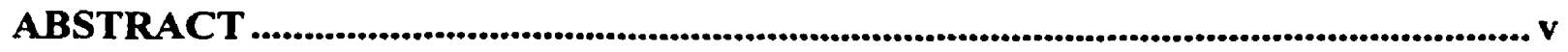

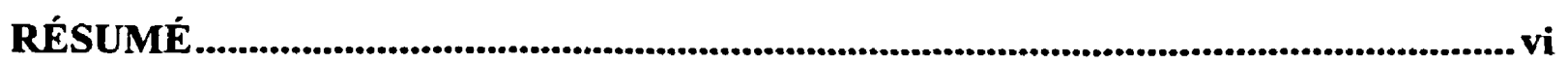

LIST OF FIGURES …...........................................................................................................

LIST OF TABLES ...............................................................................................................

LIST OF SYMBOLS AND ABBREVIATIONS .........................................................xiii

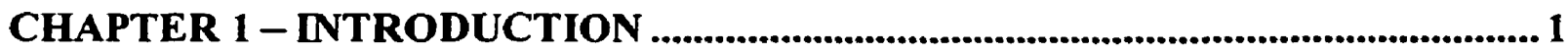

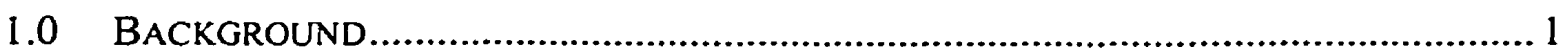

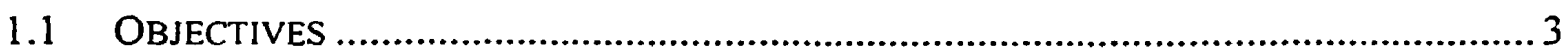

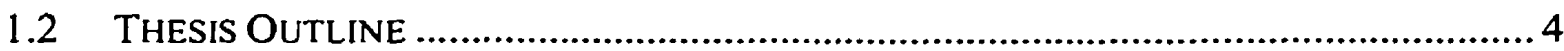

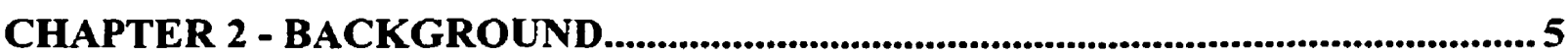

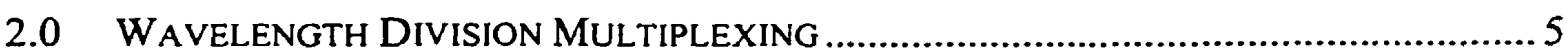

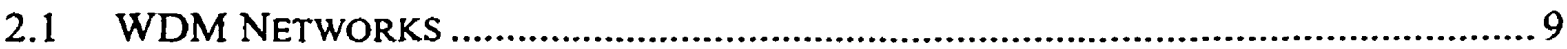

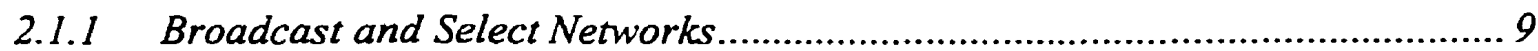

2.1.2 Wavelength Routing Networks .............................................................. 12

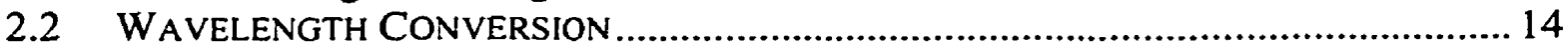

2.3 WAVELENGTH CONVERSION TECHNOLOGIES ...................................................... 18

2.3.1 Opto-Electronic Wavelength Conversion................................................. 18

2.3.2 All-Optical Wavelength Conversion ........................................................... 19

2.4 WAVELENGTH CONVERTIBLE NETWORKS ........................................................24

2.4.1 Dedicated Wavelength Convertible Switch ................................................ 25

2.4.2 Share-Per-Node Wavelength Convertible Switch …………………............ 26

2.4.3 Share-Per-Link Wavelength Convertible Switch........................................... 27

2.4.4 Electronic Wavelength Convertible Switch................................................ 28

2.4.5 Wavelength Convertible Switch Summary ................................................... 30

2.5 ROUTING AND WAVELENGTH ASSIGNMENT......................................................30

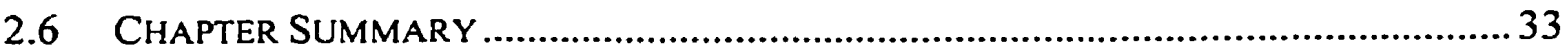

CHAPTER 3 - THE HALF-CLEAR WAVELENGTH CONVERTIBLE SWITCH AND ITS COMPARISON WITH OTHER ARCHITECTURES ................................34

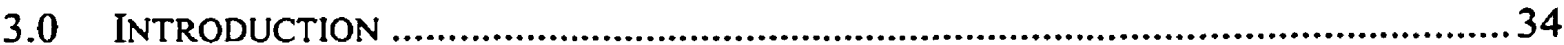

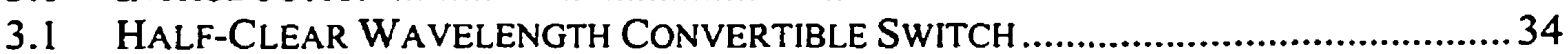




\section{TABLE OF CONTENTS, CONTINUED}

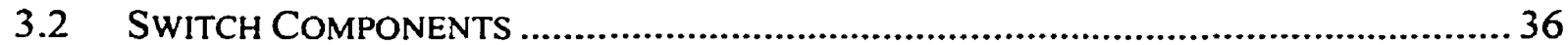

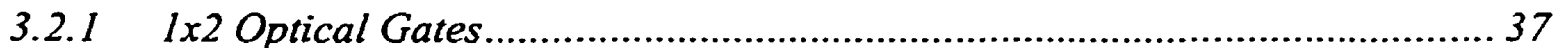

3.2.2 Non-Blocking Optical Switch............................................................. 38

3.2.3 Wavelength Multiplexer / Demultiplexer ............................................... 39

3.2.4 Wavelength Converter......................................................................... 40

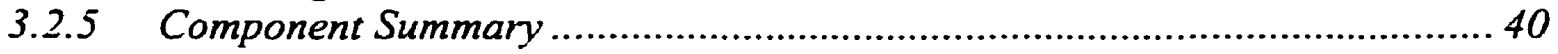

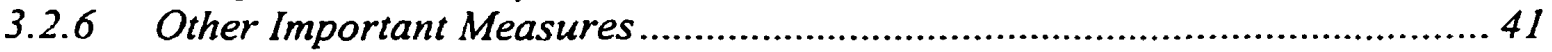

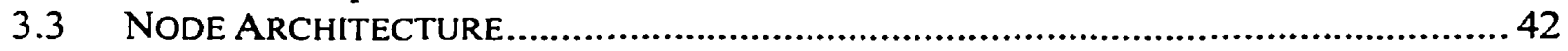

3.3.1 The Optical Switching Matrix ............................................................. 43

3.3.2 Interfacing Part........................................................................... 46

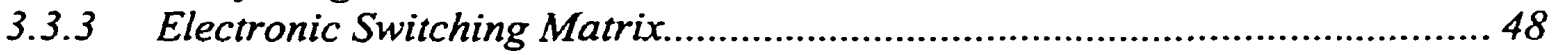

3.4 COMPARISON OF WA VELENGTH CONVERTIBLE SWITCHES ..............................48

3.4.1 The Dedicated Wavelength Convertible Switch ........................................... 49

3.4.2 The Share-Per-Node Wavelength Convertible Switch ...............................50

3.4.3 The Share-Per-Link Wavelength Convertible Switch................................. 51

3.4.4 The Electronic Wavelength Convertible Switch....................................... 54

3.4.5 The Half-Clear Wavelength Convertible Switch ....................................... 54

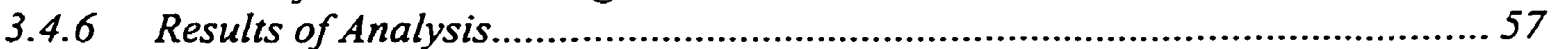

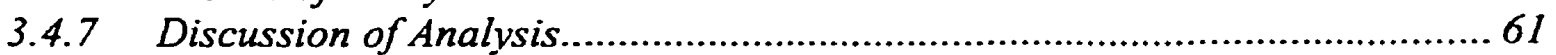

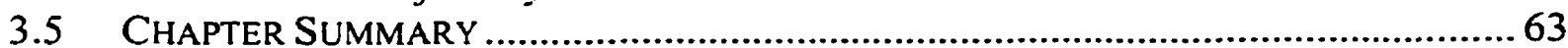

CHAPTER 4 - THE WAVELENGTH CONVERTIBLE OPTICAL NETWORK SIMULATOR (WCONSIM) ............................................................................64

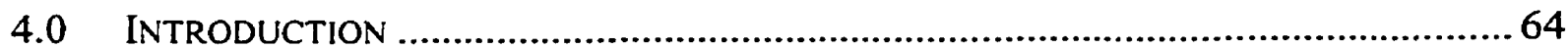

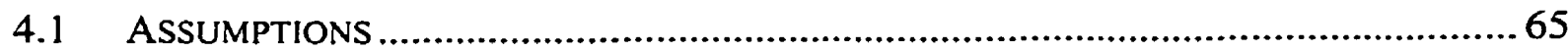

4.2 HIGH LEVEL DESCRIPTION OF SIMULATOR ............................................... 68

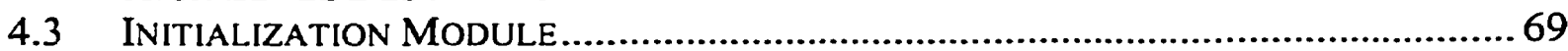

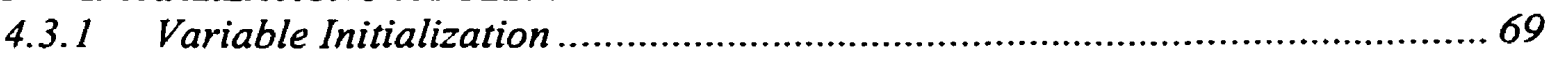

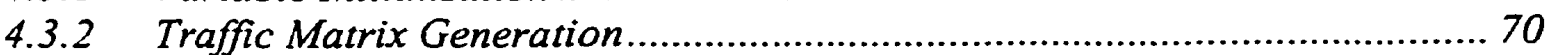

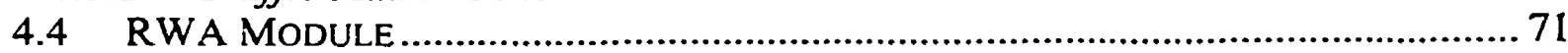

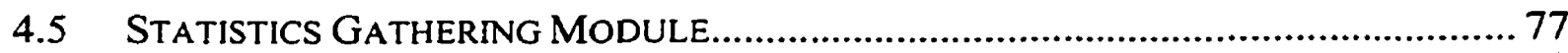

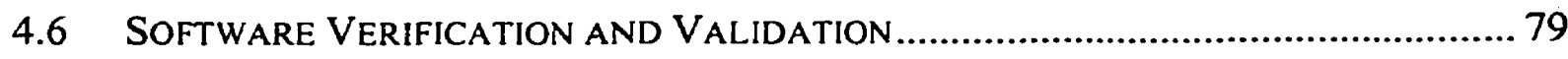

CHAPTER 5 - NUMERICAL RESULTS AND ANALYSIS ................................... 82

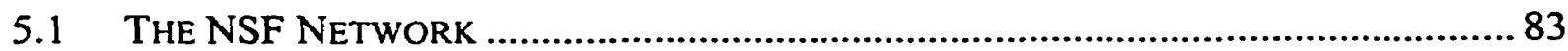

5.2 COMPARISON OF SWITCHES IN THE NSF NETWORK ................................... 103

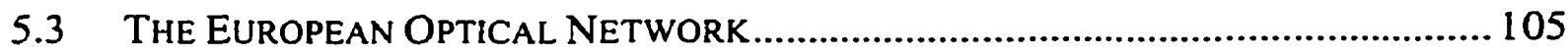

5.4 COMPARISON OF SWITCHES IN THE EON ................................................ 126 
TABLE OF CONTENTS, CONTINUED

Page

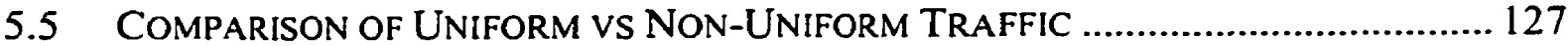

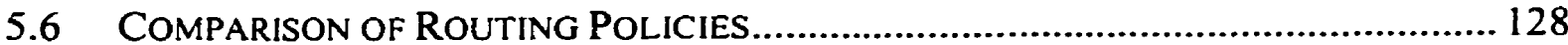

5.6.1 Routing Policy 1 vs Routing Policy 2 ............................................... 128

5.6.2 Routing Policy 3 vs Routing Policy 4................................................... 129

5.6.3 Routing Policy 2 vs Routing Policy 4............................................... 130

CHAPTER 6 - CONCLUSIONS AND FUTURE RESEARCH............................ 131

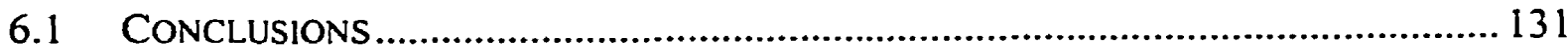

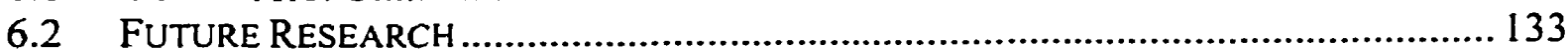

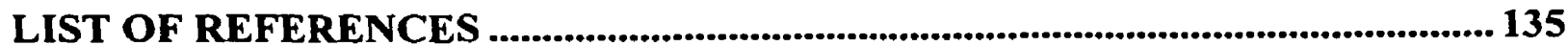

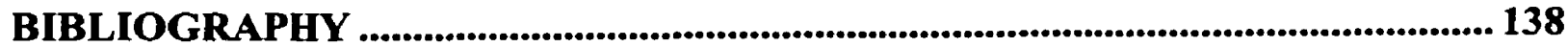

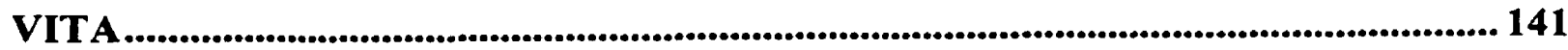




\section{LIST OF FIGURES}

Figure

Page

FIGURE 2-1: LOW ATTENUATION REGIONS OF A FIBRE OPTIC CABLE ................................. 6

FIGURE 2-2: BROADCAST-AND-SELECT NETWORK EXAMPLE .............................................. 10

FIGURE 2-3: WAVELENGTH ROUTING NETWORK EXAMPLE............................................ 13

FIGURE 2-4: WDM INTERCONNECTION NETWORK EXAMPLE .......................................... 13

FIGURE 2-5: AN ALL-OPTICAL WAVELENGTH ROUTED NETWORK...................................... 14

FIGURE 2-6: WAVELENGTH-CONTINUITY CONSTRAINT ……......................................... 16

FIGURE 2-7: FUNCTIONALITY OF A WAVELENGTH CONVERTER. ......................................... 17

FIGURE 2-8: AN OPTO-ELECTRONIC WAVELENGTH CONVERTER ........................................ 18

FIGURE 2-9: A CONVERTER BASED ON NONLINEAR WAVE-MIXING EFFECTS........................20

FIGURE 2-10: A WAVELENGTH CONVERTER USING AN SOA IN XGM MODE .......................22

FIGURE 2-11: A WAVELENGTH CONVERTER USING AN SOA IN XPM MODE ........................22

FIGURE 2-12: CONVERSION USING SATURABLE ABSORPTION IN A LASER ………………........23

Figure 2-13: A Dedicated WaVelength Convertible SwITCH...................................25

Figure 2-14: A ShaRe-PER-Node WAVELENGTH ConVERTIBLE SwITCH.........................26

Figure 2-15: A SHARE-PER-LiNK WAVELENGTH CONVERTIBLE SWITCH .........................22

Figure 2-16: AN Electronic WaVElength Convertible SWITCH ..............................28

Figure 3-1: A Half-Clear WaVelength Convertible SWITCH ................................35

FIGURE 3-2: SINGLE FIBRE ARCHITECTURE ………................................................ 43

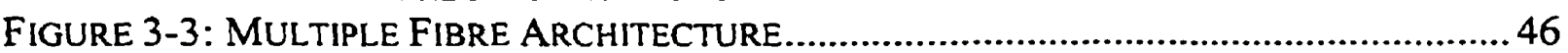

Figure 3-4: DETAIL OF A DEDICATED WAVELENGTH CONVERTIBLE SWITCH .....................50

Figure 3-5: Detail of a Share-Per-Node WaVElength ConverTible SWITCH .........52

Figure 3-6: DetaIl OF THE SHARE-PER-LinK WAVELENGTH CONVERTIBLE SWITCH .......53

Figure 3-7: DeTAIL OF THE Electronic WAVELENGTH CONVERTIBLE SWITCH ..............55

Figure 3-8: Detail of THE Half-Clear WaVElength Convertible SWITCH................56

FIGURE 3-9: COMPONENT COSTS OF OPTICAL SWITCHES, $\Delta_{p}=4, \mid m=16 \ldots \ldots \ldots \ldots \ldots \ldots \ldots . .58$

FIGURE 3-10: INCREMENTAL COSTS ASSOCIATED WITH $\Delta_{p},|W|=16 \ldots \ldots \ldots \ldots \ldots \ldots \ldots \ldots \ldots . . . .59$

FIGURE 3-11: INCREMENTAL COSTS ASSOCIATED WITH $\mid W, \Delta_{P}=4 \ldots \ldots \ldots \ldots \ldots \ldots \ldots \ldots \ldots \ldots . . . . . .59$

FIGURE 3-12: DB LOSS FOR A PACKET TO PASS THROUGH A SWITCH …...........................60

FIGURE 3-13: PROCESSING TIME FOR A PACKET TO PASS THROUGH A SWITCH....................60

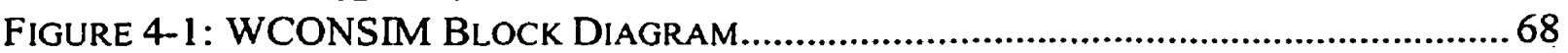

FigURE 4-2: ILLUSTRATION OF A CONTINUOUS CLEAR LIGHTPATH .................................72

FigURE 4-3: ILLUSTRATION OF A NON-CONTINUOUS CLEAR LIGHTPATH ............................73

FIGURE 4-4: ILLUSTRATION OF AN UNCLEAR LIGHTPATH ................................................ 74

FIGURE 4-5: THE 7-NODE TEST NETWORK AND ITS DISTANCE MATRIX ................................8 80

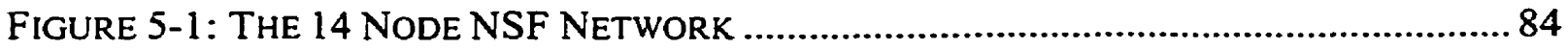

FIGURE 5-2: NSF NETWORK/UNIFORM TRAFFIC - NETWORK COSTS VS |W|.......................85

FIGURE 5-3: NSF NETWORK/NON-UNIFORM TRAFFIC - NETWORK COSTS VS |W|.............. 86 


\section{LIST OF FIGURES, CONTINUED}

Figure

Page

FIGURE 5-4: NSF/UNIFORM TRAFFIC - THROUGHPUT VS |W| FOR POLICY 1 87

FIGURE 5-5: NSF /UNIFORM TRAFFIC - THROUGHPUT VS |W| FOR POLICY $2 \ldots \ldots \ldots \ldots \ldots \ldots . . . . . . .88$

FIGURE 5-6: NSF /UNIFORM TRAFFIC - THROUGHPUT VS |W| FOR POLICY 3 ...................89

FIGURE 5-7: NSF /UNIFORM TRAFFIC - THROUGHPUT VS |W| FOR POLICY 4 ...................90

FIGURE 5-8: NSF /UNIFORM TRAFFIC - DELAY VS |W| FOR POLICY 1 ...........................91

FIGURE 5-9: NSF /UNIFORM TRAFFIC - DELAY VS |W| FOR POLICY $2 \ldots \ldots \ldots \ldots \ldots \ldots \ldots \ldots . . . \ldots 92$

FIGURE 5-10: NSF /UNIFORM TRAFFIC - DELAY VS |W| FOR POLICY 3 .........................93

FIGURE 5-1 I : NSF /UNIFORM TRAFFIC - DELAY VS |W| FOR POLICY 4.......................... 94

FIgURE 5-12: NSF NON-UNIFORM TRAFFIC - THROUGHPUT VS |W| FOR POLICY 1 ......... 95

FIgURE 5-13: NSF / NON-UNIFORM TRAFFIC - THROUGHPUT VS |W| FOR POLICY 2 ........ 96

FIGURE 5-14: NSF / NON-UNIFORM TRAFFIC - THROUGHPUT VS |W| FOR POLICY 3 ........ 97

FIGURE 5-15: NSF / NON-UNIFORM TRAFFIC - THROUGHPUT VS |W| FOR POLICY 4 .........98

FIGURE 5-16: NSF / NON-UNIFORM TRAFFIC - DELAY VS |W| FOR POLICY 1 .................99

FIGURE 5-17: NSF / NON-UNIFORM TRAFFIC - DELAY VS |W| FOR POLICY 2 ............... 100

FIGURE 5-18: NSF / NON-UNIFORM TRAFFIC - DELAY VS |W| FOR POLICY 3 ................. 101

FIGURE 5-19: NSF / NON-UNIFORM TRAFFIC - DELAY VS |W| FOR POLICY 4 ................. 102

FIGURE 5-20: THE 19-NODE EON WIDE-AREA NETWORK …..................................... 107

FIGURE 5-21: EON NETWORK/UNIFORM TRAFFIC - NETWORK COSTS VS |W|.............. 108

FIGURE 5-22: EON NETWORK/NON-UNIFORM TRAFFIC - NETWORK COSTS VS |W|....... 109

FIGURE 5-23: EON /UNIFORM TRAFFIC - THROUGHPUT VS |W| FOR POLICY 1 .............. 110

FIGURE 5-24: EON /UNIFORM TRAFFIC - THROUGHPUT VS |W| FOR POLICY $2 \ldots \ldots \ldots \ldots \ldots 111$

FIGURE 5-25: EON /UNIFORM TRAFFIC - THROUGHPUT VS |W| FOR POLICY $3 \ldots \ldots \ldots \ldots . . . . .112$

FIGURE 5-26: EON /UNIFORM TRAFFIC - THROUGHPUT VS |W| FOR POLICY 4 ............... 113

FIGURE 5-27: EON /UNIFORM TRAFFIC - DELAY VS |W| FOR POLICY 1 ........................ 114

FIGURE 5-28: EON /UNIFORM TRAFFIC - DELAY VS |W| FOR POLICY 2 ....................... 115

FIGURE 5-29: EON /UNIFORM TRAFFIC - DELAY VS |W| FOR POLICY 3 ......................... 116

FIGURE 5-30: EON /UNIFORM TRAFFIC - DELAY VS |W| FOR POLICY 4 ........................ 117

FIGURE 5-31: EON $/$ NON-UNIFORM TRAFFIC - THROUGHPUT VS |W| FOR POLICY $1 \ldots . . .118$

FIGURE 5-32: EON / NON-UNIFORM TRAFFIC - THROUGHPUT VS $\mid$ W| FOR POLICY $2 \ldots . . .119$

FIGURE 5-33: EON / NON-UNIFORM TRAFFIC - THROUGHPUT VS |W| FOR POLICY $3 \ldots 120$

FIGURE 5-34: EON / NON-UNIFORM TRAFFIC - THROUGHPUT VS |W| FOR POLICY 4 ..... 121

FIGURE 5-35: EON / NON-UNIFORM TRAFFIC - DELAY VS |W| FOR POLICY 1 ................ 122

FIGURE 5-36: EON / NON-UNIFORM TRAFFIC - DELAY VS |W| FOR POLICY 2 ................ 123

FIGURE 5-37: EON / NON-UNIFORM TRAFFIC - DELAY VS |W| FOR POLICY 3 ................ 124

FIGURE 5-38: EON / NON-UNIFORM TRAFFIC - DELAY VS |W| FOR POLICY 4 .............. 125 


\section{LIST OF TABLES}

Table

Page

TABLE 2-1: WAVELENGTH CONVERTIBLE SWITCH SUMmaRY ................................... 30

TABle 3-1: WaVElength Convertible Switch Summary ..................................... 36

TABLE 3-2 : SUMMARY OF COMPONENT CHARACTERISTICS .................................... 41 


\section{LIST OF SYMBOLS AND ABBREVIATIONS}

$\begin{array}{ll}\text { BAS } & \text { Broadcast and Select Networks } \\ \text { CDM } & \text { Code Division Multiplexing } \\ \text { CDMA } & \text { Code Division Multiple Access } \\ \text { CW } & \text { Continuous Wave } \\ \text { DED } & \text { Dedicated Wavelength Convertible Switch } \\ \text { DFG } & \text { Difference Frequency Generation } \\ \text { DWDM } & \text { Dense Wavelength Division Multiplexing } \\ \text { ELE } & \text { Electronic Wavelength Convertible Switch } \\ \text { E/O } & \text { Electronic / Optical Conversion } \\ \text { EON } & \text { European Optical Network } \\ \text { FDDI } & \text { Fibre Distributed Data Interface } \\ \text { FWM } & \text { Four Wave Mixing } \\ \text { HALF } & \text { Half-Clear Wavelength Convertible Switch } \\ \text { MILP } & \text { Mixed Integer Linear Problem (mathematical) } \\ \text { MZI } & \text { Mach-Zender Interferometer } \\ \text { NSF } & \text { National Science Foundation } \\ \text { OCDM } & \text { Optical Code Division Multiplexing }\end{array}$


LIST OF SYMBOLS AND ABBREVIATIONS, CONTINUED

$\mathrm{O} / \mathrm{E}$

OSW

OTDM

RWA

RWA/NCO

SDM

SDMA

SPN

SPL

SOA

TDM

TDMA

WCONSIM

WDM

WDMA

WRN

XGM
Optical / Electronic Conversion

Optical Switch

Optical Time Division Multiplexing

Routing and Wavelength Assignment

Routing and Wavelength Assignment /

Network Cost Optimization

Space Division Multiplexing

Space Division Multiple Access

Share-Per-Node Wavelength Convertible Switch

Share-Per-Link Wavelength Convertible Switch

Semiconductor Optical Amplifier

Time Division Multiplexing

Time Division Multiple Access

Wavelength Convertible Optical Network Simulator

Wavelength Division Multiplexing

Wavelength Division Multiple Access

Wavelength Routed Networks

Cross Gain Modulation 


\section{LIST OF SYMBOLS AND ABBREVIATIONS, CONTINUED}

$\begin{array}{ll}\text { XPM } & \text { Cross Phase Modulation } \\ |\mathrm{W}| & \text { Number of Wavelengths } \\ f_{c} & \text { Converted signal frequency } \\ f_{p} & \text { Pump signal frequency } \\ f_{s} & \text { Input signal frequency } \\ \lambda_{c} & \text { Converted signal wavelength } \\ \lambda_{p} & \text { Pump signal wavelength } \\ \lambda_{s} & \text { Input signal wavelength }\end{array}$

Following symbols are used in the simulation software (Chapter 4)

$\Delta_{\mathrm{p}} \quad$ Physical degree of the node

$\Lambda \quad$ Traffic matrix

$\underline{Y} \quad$ Matrix whose entries indicating s-d pairs whose traffic has been successfully routed

$\underline{\mathrm{V}}^{\mathrm{k}} \quad$ Virtual connectivity on wavelength $\lambda_{\mathrm{k}}$

$\hat{\lambda}_{i j} \quad$ Aggregate traffic on link $l_{i j}$

$\bar{\lambda}_{i} \quad$ Number of wavelength converters in use at node $i$ 


\section{CHAPTER 1 - INTRODUCTION}

\section{$1.0 \quad$ Background}

Today's society is increasingly dependent on information. Traditionally, this information is being provided through a global array of computer networks. Looking back, these networks can be categorized into three generations [1]:

- First generation networks built before the emergence of fibre technology, that is, based on copper-wire or radio based technologies. For example, ARPANET.

- Second generation networks that employ fibre in traditional architectures. Mostly employing fibre optics in long haul trunks over a wide area network. For example, SONET.

- Third generation networks that provide services such as "fibre to the desktop" are expected to provide very high bandwidth to users through the use of alloptical switching and intermediate processing capabilities.

It is anticipated, that as companies, universities and research facilities continue to upgrade their computing infrastructure, the need to interconnect an ever increasing number of high performance bandwidth hungry workstations will become apparent. For example, the medical imaging community, which does not trust image-compression techniques, will require the transmission of uncompressed images. With these applications, and many others, it is anticipated that the end user will generate a sustained 
bandwidth demand of approximately $1 \mathrm{~Gb} / \mathrm{s}$ [1]. Networks employing electronic switching and processing will not be sufficient to handle these demands.

Today, networks speeds are still limited by the electronic speed (usually a few $\mathrm{Gb} / \mathrm{s}$ ) of the terminating equipment. To tap into the huge bandwidth available in lightwave networks, multiple access techniques are introduced into the network routing protocols and architectures. Wavelength Division Multiplexing (WDM) has emerged as the optimum technology to utilize the bandwidth available in lightwave networks. This technique allows different end-users to operate at electronic speeds, but multiple WDM channels from different end users may be multiplexed on the same fibre [2]. There are two types of networks that employ WDM techniques: broadcast-and-select networks, and wavelength-routed networks. These are explained in detail in Chapter 2.

In a wavelength-routed network, a call can only proceed if the same wavelength is available on all the links in the path. This is called the wavelength continuity constraint [2]. With this constraint, the network is susceptible to higher call blocking probabilities than regular circuit switched networks. It is therefore beneficial to relax the wavelength continuity constraint and allow the wavelength of a call to be converted at intermediate nodes where wavelength contention occurs.

Wavelength converters are expensive pieces of equipment. It is therefore necessary to configure optical switches to maximize the benefits of wavelength conversion, while keeping costs to a minimum. There are five optical switch architectures common in today's switch designs that use wavelength converters [2]: 
- a dedicated wavelength-convertible switch,

- a share-per-node wavelength convertible switch,

- a share-per-link wavelength convertible switch,

- an electronic wavelength convertible switch.

Many of these architectures have been reviewed and analyzed in the literature under specific conditions using various metrics [3-5]. A review of these switches under the same conditions, with the same metrics, has yet to be done.

Dedicated wavelength convertible switches offer the most flexibility since all output signals have the capability of being converted. It is, however, very costly due to the number of converters used in this architecture. This leads to the development of other architectures (such as share-per-node and share-per-link) where the primary purpose is to share the converters to reduce the cost. This thesis will also introduce another novel design, the half-clear wavelength convertible switch, which is a compromise between performance and cost.

\section{$1.1 \quad$ Objectives}

The objectives of this thesis are summarized below.

- To investigate the use of wavelength converters within an optical switch by considering individual component characteristics within each switch architecture.

- To design a new wavelength convertible switch to compromise between cost and performance. 
- To investigate the performance of the new wavelength convertible switch compared with the full conversion case and the no conversion case.

\subsection{Thesis Outline}

The remainder of this thesis will be broken down into the following chapters. Chapter 2 discusses background material in optical networking, wavelength division multiplexing, wavelength conversion technologies, and wavelength convertible switches. It also overviews previous work that has been done in this area of research. Chapter 3 presents a comparison of the five switches by investigating their individual component characteristics. Cost, attenuation and packet delay are investigated in this chapter. In Chapter 4 the Wavelength Convertible Optical Switch Simulator (WCONSIM) is introduced. This simulator is used in evaluating the half-clear wavelength convertible switch. Chapter 5 presents and discusses the numerical results obtained from simulation. Finally, Chapter 6 summarizes the findings and suggests some future research areas in this field of research. 


\section{CHAPTER 2 - BACKGROUND}

\subsection{Wavelength Division Multiplexing}

Although aggregate bandwidth demands are increasing for today's networks, end users are still limited to the processing speeds of the electronic terminating equipment. This leads to exploring methods of providing more bandwidth. To exploit a fibre cable's huge bandwidth, network designers turn to multiplexing techniques to set up concurrent connections over the same physical cable plant. Time Division Multiplexing (TDM), Space Division Multiplexing (SDM), Code Division Multiplexing (CDM) or Wavelength Division Multiplexing (WDM) techniques can achieve this concurrency.

Space Division Multiplexing (SDM) is the most straightforward approach. For fibre optic links, it uses more fibre links along each path to allow more connections to be made. Although this approach is simple, it is not always feasible due to the cost of laying new fibre cable. Time Division Multiplexing (TDM) and Code Division Multiplexing (CDM) share the transport medium by subdividing time slots (in the case of TDM) or making use of a properly constructed code (in the case of CDM). Optical TDM and CDM (OTDM / OCDM) are still in the research stage and are a few years away from implementation. Wavelength Division Multiplexing (WDM) has emerged as the optimum technology to utilize the bandwidth available in lightwave networks. 
With wavelength division multiplexing, the optical transmission spectrum is divided into two regions of low loss wavebands, each approximately $200 \mathrm{~nm}$ wide. These two regions provide an aggregate bandwidth of approximately $50 \mathrm{THz}$ (see Figure 2-1) [2]. This translates into many orders of magnitude higher than the peak electronic data rate.

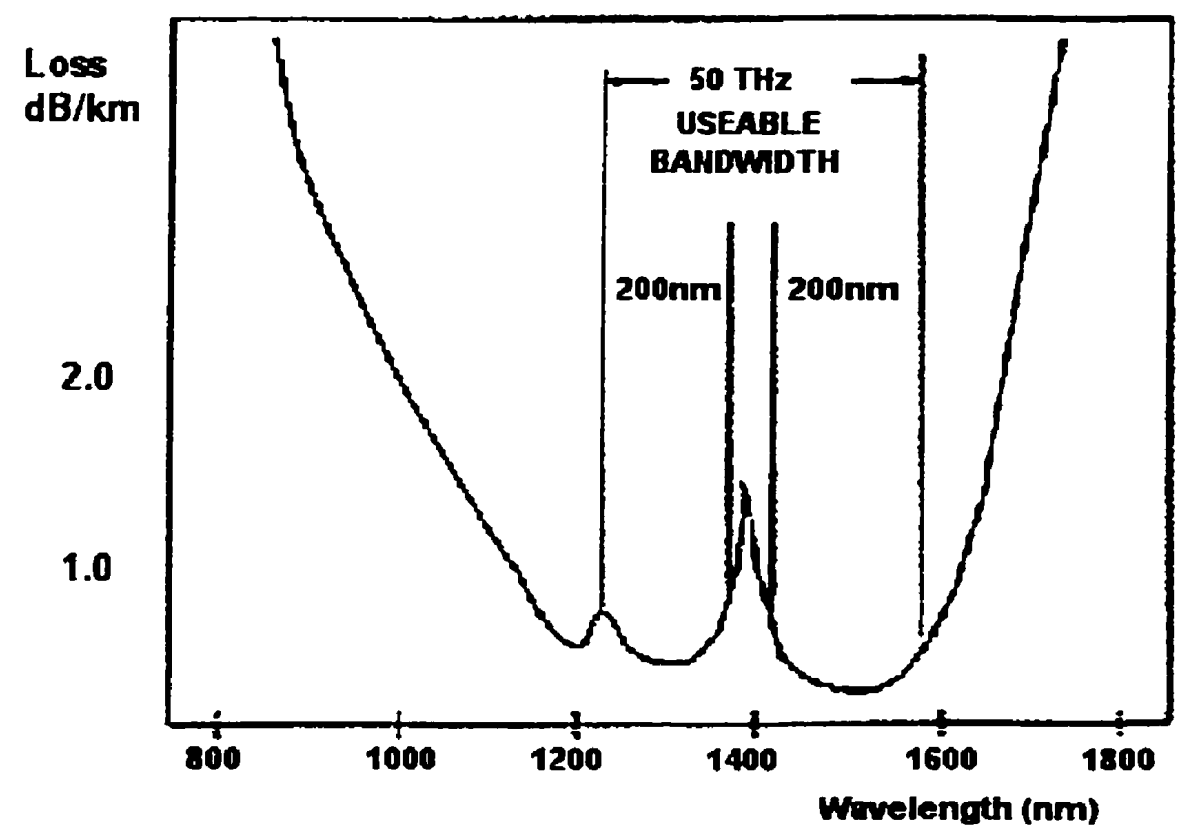

Figure 2-1: Low attenuation regions of a fibre optic cable [2]

The WDM approach allows different end-users to operate at electronic speeds, but multiple WDM channels from different end-users may be multiplexed on the same fibre [2]. This approach has many advantages over the other multiplexing techniques discussed earlier for the following reasons: 
- A lower bit rate is used in WDM systems; thus, the distance limits imposed by dispersion (chromatic and polarization) are larger than in TDM systems.

- The transmission capacity can be increased in a modular manner with WDM systems. For example, a four-channel WDM system can have the transmission capacity modularly increased from $2.5 \mathrm{~Gb} / \mathrm{s}$ up to $10 \mathrm{~Gb} / \mathrm{s}$. The same TDM system would require the full $10 \mathrm{~Gb} / \mathrm{s}$ capacity to be installed all at once.

- WDM systems can be configured to be transparent. This means that different channels can operate at different bit rates. In some circumstances, this can be a major advantage.

- In the overall network, some nodes may have a significant amount of traffic pass through them, with very little added or dropped. In this case, WDM networking may be more cost effective to use WDM add/drop components in these specific nodes than using other multiplexing terminals.

On the other hand, there are a number of disadvantages with WDM that need mention:

- A WDM system requires separate terminating equipment for each channel (transmitter and receiver). Whereas, TDM systems only require one piece of terminating equipment. 
- WDM transparency can make network management and monitoring more difficult than in TDM systems. With multiple channels operating at different bit rates and format, the network cannot monitor bit error rates and frame errors. This capability would have to be done in the terminating equipment. However, this makes fault isolation more difficult as it may be hard to determine if the fault is in the WDM equipment or in the terminating equipment.

- WDM systems require specially designed optical amplifiers that must provide gain independent of the number of wavelengths in the system. This is especially important to ensure that a failure in one channel does not cause failures in other channels.

Based on this discussion, network designers are faced with the question of what type of multiplexing technique they should use. Should they deploy a WDM system with 32 wavelengths at $2.5 \mathrm{~Gb} / \mathrm{s}$ or use TDM as well and have a system with 8 wavelengths operating at $10 \mathrm{~Gb} / \mathrm{s}$ each? Each option has its merits and downfalls and a tradeoff is necessary when designing networks. A combination of WDM and TDM is likely to become the standard in the future. 


\section{$\underline{2.1 \quad \text { WDM Networks }}$}

A WDM network is traditionally made up of a number of network access nodes interconnected by a shared optical medium. The network access nodes perform two main tasks: First, the node converts signals from the electrical domain to the optical domain (E/O conversion) and from the optical domain back to the electrical domain (O/E conversion). Second, the node places traffic into the optical medium, and retrieves traffic from the optical medium. The node can also perform routing and switching functions depending on the network in question.

WDM networks can be broadly classified into two main categories based on the optical medium in use within the network. These two categories are: broadcast-andselect (BAS) networks and wavelength-routing networks (WRN) [1, 2].

\subsubsection{Broadcast and Select Networks}

In this type of network, all inputs are combined in a star coupler and broadcast to all outputs. Figure 2-2 shows an example of this network. The broadcast star illustrates the combining and splitting functionality of the network.

A number of variations on this network exist, depending on whether the transmitters and receivers are fixed or tunable. There are four possibilities: 


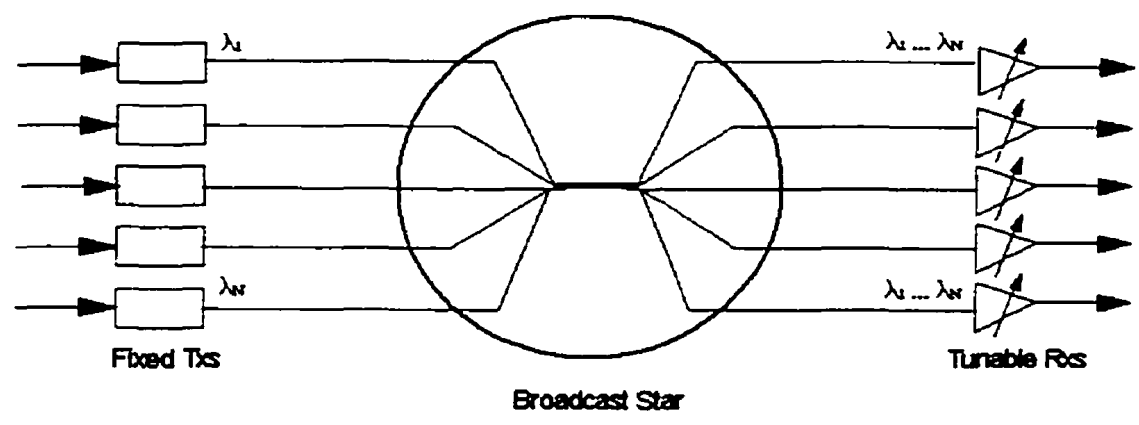

Figure 2-2: Broadcast-and-Select Network Example

- fixed transmitters - fixed receivers (FT-FR),

- fixed transmitters - tunable receivers (FT-TR),

- tunable transmitters - fixed receivers (TT-FR), and

- tunable transmitters, tunable receivers (TT-TR).

A fixed transmitter - fixed receiver (FT - FR) system is generally suited for a multihop network where no dynamic system reconfiguration is necessary, although single hop systems with a small number of nodes have been demonstrated (LAMBDANET) [ 1 2].

Systems employing fixed receivers (FT-FR, TT-FR) do not require any control channel selection coordination. Each node is assigned a single channel eliminating collisions. The disadvantage is that the maximum number of nodes is limited to the number of channels available in the system. 
The TT-TR structure is the most flexible and can easily accommodate a scalable user population. This is at the cost of increased overhead and control channel coordination for transmitters.

Flexible systems can be constructed with each node having a combination of transmitters and receivers. The following general classification for these networks can be developed [1 - 2]:

$\left\{\begin{array}{l}F T^{i} T T^{j}-F R^{m} T R^{n} \quad \text { No pretransmission coordination } \\ C C-F T^{i} T T^{j}-F R^{m} T R^{n} \quad \text { Control-channel }(C C) \text {-based system }\end{array}\right.$

where a node has $i$ fixed transmitters, $j$ tunable transmitters, $m$ fixed receivers and $n$ tunable receivers.

A broadcast-and-select network has the following advantages:

- The switching function is centralized. This provides inherent fault tolerance. If a node is down, the others can still function.

- No additional functionality is required for multicasting. Nodes intended as recipients for a multicast packet need only tune their receivers to the same wavelength.

- This type of network is inherently cheap to construct. The broadcast-andselect star is mostly glass and cheap to construct. No expensive switching elements are required. 
This type of network also has disadvantages:

- The number of wavelengths in the network must be equal to the number of nodes. This limits the maximum size of the network.

- The number of nodes in the network is also limited since the transmitted power from a node must be split between all receivers in the network.

- Synchronization and multiple access techniques become more crucial in broadcast-and-select networks to avoid potential collisions.

\subsubsection{Wavelength Routing Networks}

Wavelength Routing Networks (WRN) are made up of a number of wavelengthselective elements and route signals based on their wavelength and the port they entered the network. An example of such a network is shown in Figure 2-3.

In the network shown in Figure 2-3, there are $N$ tunable transmitters interconnected with $N$ receivers through a number of WDM elements. By tuning the transmitter to a specific wavelength, the signal can be routed to the intended receiver in the network. One would expect in an $N x N$ network, $N^{2}$ wavelengths would be required. However, this is not the case. The signals can be interconnected in such a way that only $N$ wavelengths are required. This is shown in Figure 2-4. In Figure 2-4b the wavelength assignments for the $4 \times 4$ network are shown. Only four unique wavelengths are required with this connectivity. 


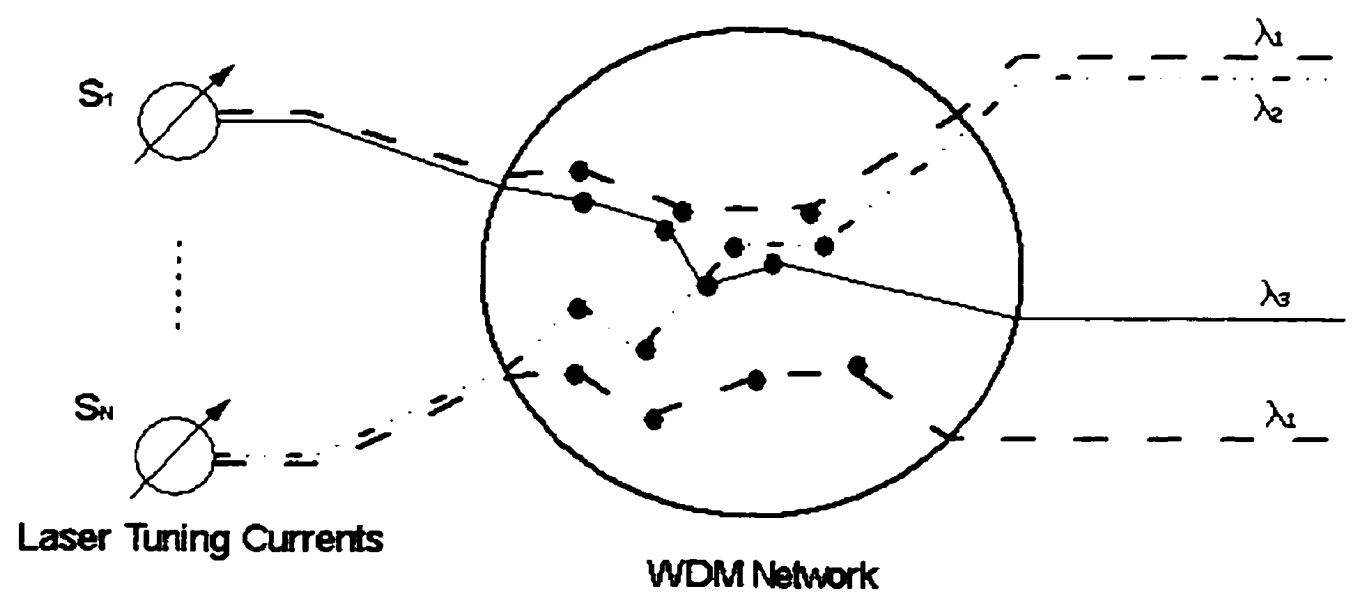

Figure 2-3: Wavelength Routing Network Example

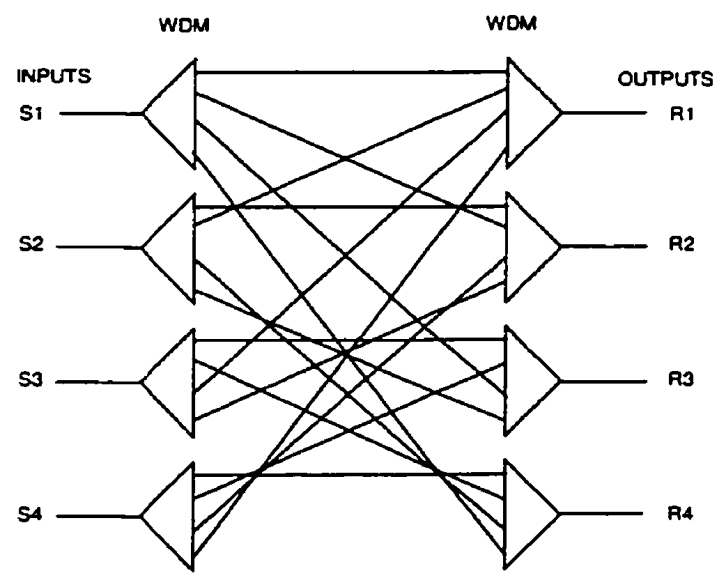

a

\begin{tabular}{|c|c|c|c|c|}
\hline \multirow{2}{*}{ Input } & \multicolumn{4}{|c|}{ Output } \\
& $\mathrm{R} 1$ & $\mathrm{R} 2$ & $\mathrm{R} 3$ & $\mathrm{R} 4$ \\
\cline { 2 - 5 } $\mathrm{S} 1$ & $\lambda 0$ & $\lambda 1$ & $\lambda 2$ & $\lambda 3$ \\
\cline { 2 - 5 } $\mathrm{S} 2$ & $\lambda 1$ & $\lambda 0$ & $\lambda 3$ & $\lambda 2$ \\
\cline { 2 - 5 } $\mathrm{S} 3$ & $\lambda 2$ & $\lambda 3$ & $\lambda 0$ & $\lambda 1$ \\
\cline { 2 - 5 } $\mathrm{S} 4$ & $\lambda 3$ & $\lambda 2$ & $\lambda 1$ & $\lambda 0$ \\
\hline
\end{tabular}

b

Figure 2-4: WDM Interconnection Network Example 


\subsection{Wavelength Conversion}

A wavelength-routed network is shown in Figure 2-5. It has two WDM crossconnects (S1 and S2) and five access stations (A through E). The network has three lightpaths established: $\mathrm{C}$ to $\mathrm{A}$ on wavelength $\lambda_{1}, \mathrm{C}$ to $\mathrm{B}$ on $\lambda_{2}$, and $\mathrm{D}$ to $\mathrm{E}$ on $\lambda_{1}$. To establish lightpaths in a network of this type, it is beneficial to use the same wavelength on all the links in the lightpath. This requirement is known as the wavelength-continuity constraint [2]. This constraint is what distinguishes a wavelength-routed network with a circuit switched network. A circuit switched network blocks calls only when there is no capacity along one of the links in the path. Whereas a wavelength-routed network would block a call if the same wavelength were not available along all the links in the path, regardless of whether or not the capacity on the links is available. This may result in more calls being blocked in the wavelength-routed network.

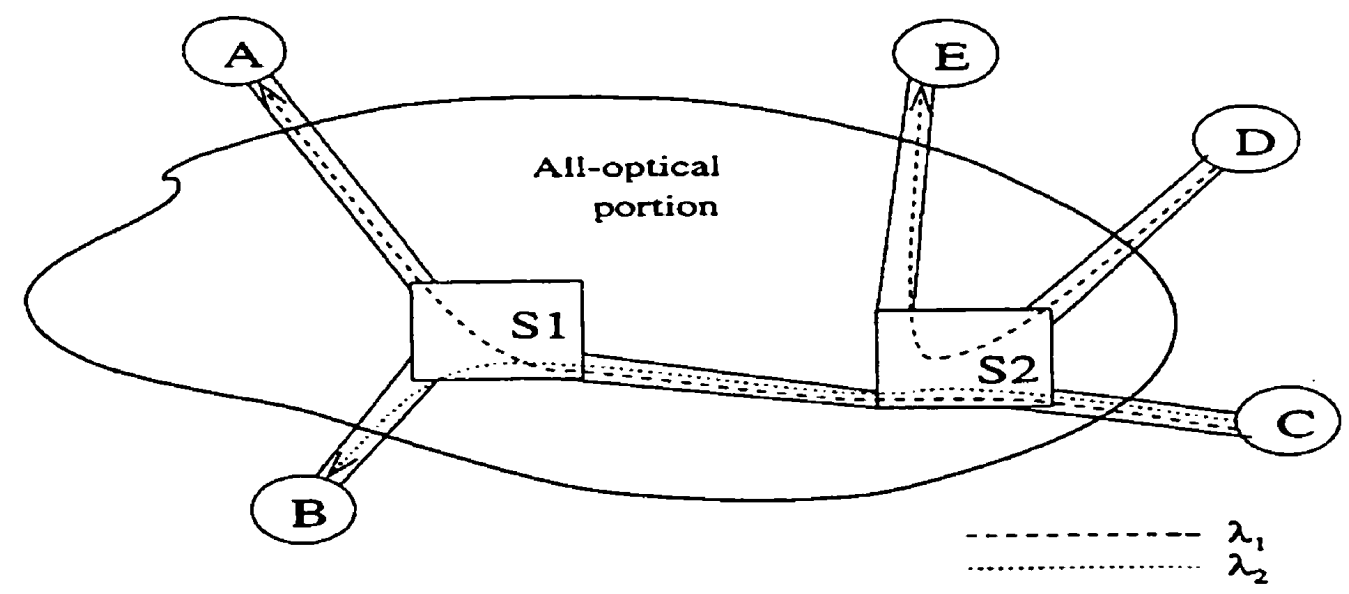

Figure 2-5: An all-optical wavelength routed network [2]. 
To improve efficiency in the wavelength-routed network, it is sometimes beneficial to relax the wavelength continuity constraint and to allow the wavelength of the lightpath to be converted at intermediate nodes. This is illustrated in Figure 2-6. In this example, two lightpaths have been previously set up. From Node 1 to Node 2, there is a connection using wavelength $\lambda_{1}$. From Node 2 to Node 3 there is a connection using wavelength $\lambda_{2}$ (Figure 2-6a). In a network where there is no wavelength conversion, a requirement to connect Node 1 to Node 3 would be blocked since there is no common wavelength available on all the links along the path, even though there is a free wavelength available on each link. With the use of a wavelength converter at Node 2, the connection can be made (Figure 2-6b). In this scenario, the lightpath between Node 1 and Node 2 utilizes wavelength $\lambda_{2}$ and the link between Node 2 and Node 3 utilizes wavelength $\lambda_{1}$. Networks of this type are often referred to as wavelength-convertible networks [2]. Networks utilizing wavelength converters have the potential for increased throughput over those without wavelength converters, since wavelength conflicts can be resolved.

The function of a wavelength converter is a relatively simple one: to convert the data on an input wavelength onto a possibly different output wavelength among the $N$ wavelengths in the system. Figure 2-7 shows this functionality. Throughout the remainder of this thesis, the following terminology is used, $\lambda_{s}$ represents the input signal wavelength, $f_{\mathrm{s}}$ represents the input signal frequency; $\lambda_{\mathrm{c}}$ represents the converted signal 


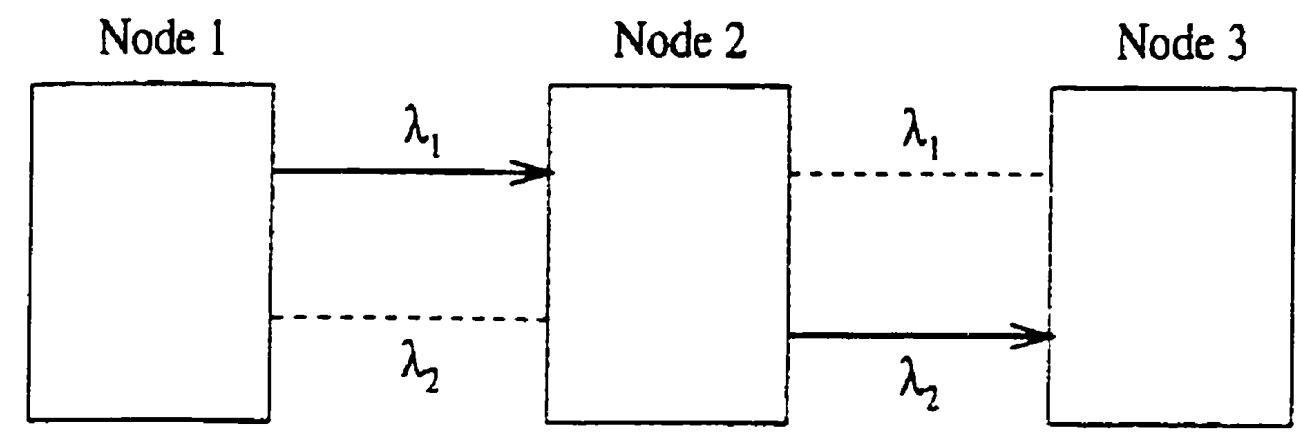

(a) without converter

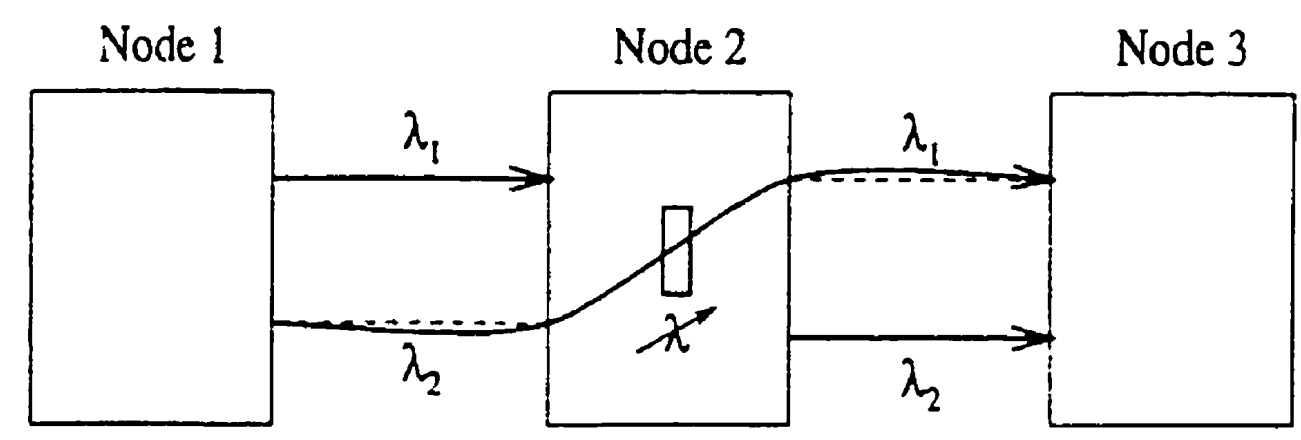

(b) with converter

Figure 2-6: Wavelength-continuity constraint [2].

wavelength, $f_{c}$ the converted signal frequency; and $\lambda_{p}$ represents the pump signal wavelength, $f_{\mathrm{p}}$ the pump signal frequency. $C W$ is the continuous wavelength signal generated as the pump signal. This signal is generally unmodulated. 


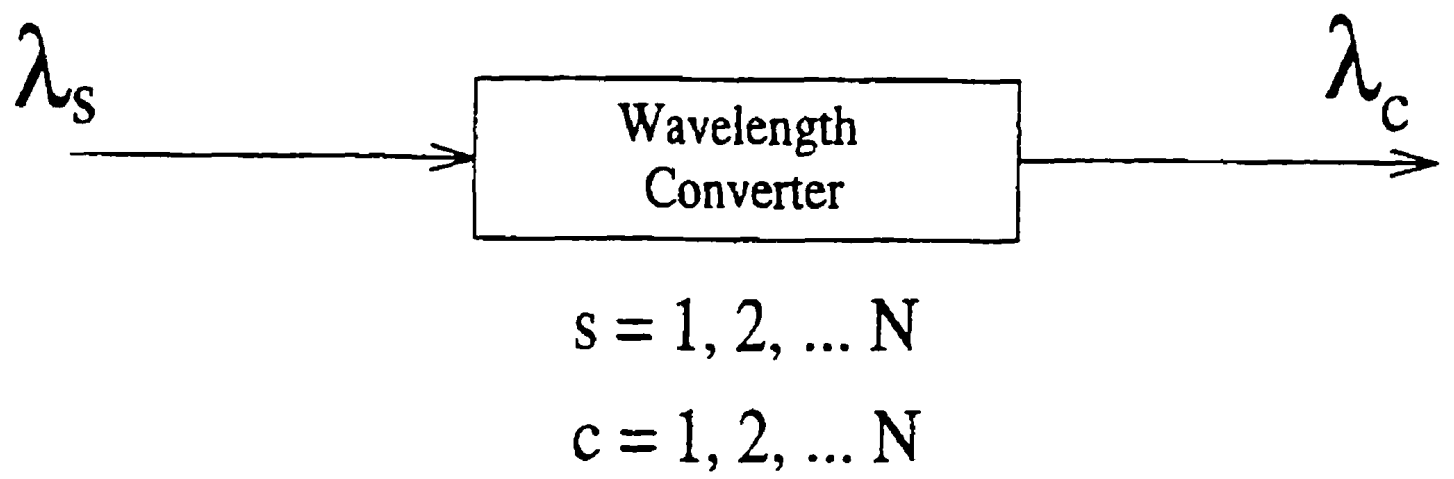

Figure 2-7: Functionality of a wavelength converter [2].

The ideal wavelength converter should possess the following characteristics [2]:

- transparency to bit rates and signal formats,

- fast setup time of output wavelength,

- conversion to both shorter and longer wavelengths,

- moderate input power levels,

- possibility for same input and output wavelengths (i.e. no conversion),

- insensitivity to input signal polarization,

- low-chirp output signal with high extinction ratio ${ }^{\prime}$ and large signal-tonoise ratio, and

- simple implementation.

\footnotetext{
'The extinction ratio is defined as the ratio of the optical power transmitted for a bit " 0 " to the power transmitted for a bit " $l$ ".
} 


\subsection{Wavelength Conversion Technologies}

Wavelength conversion techniques can be broadly classified into two groups [6]: opto-electronic wavelength conversion and all-optical wavelength conversion. Optoelectronic techniques first convert the optical signal into an electronic signal whereas alloptical techniques keep the signal in the optical domain. The following subsections describe the different wavelength conversion technologies in more detail.

\subsubsection{Opto-Electronic Wavelength Conversion}

In this type of wavelength conversion, the optical signal is converted into an electronic signal using a photodetector. The electronic signal is then buffered within the node. The electronic signal is used as the input to a tunable laser, which can be tuned to the desired wavelength of the output. (See Figure 2-8)

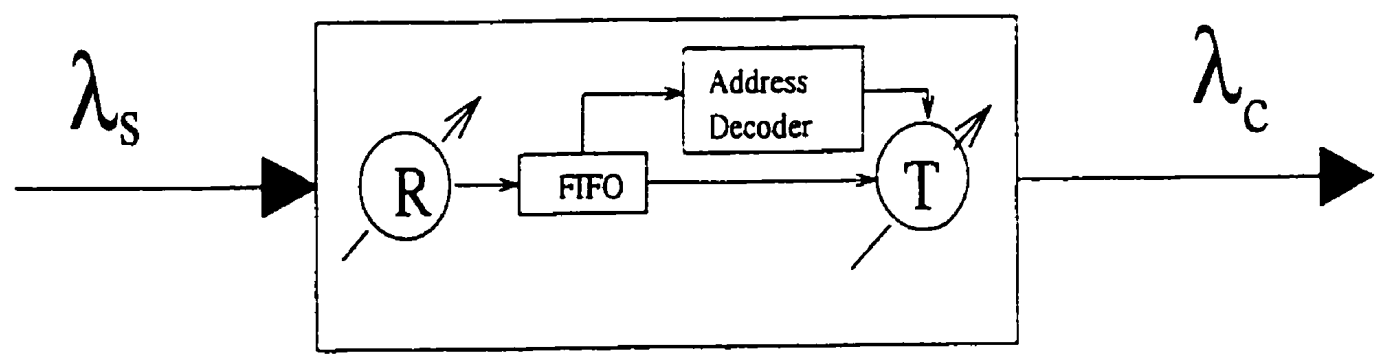

Figure 2-8: An opto-electronic wavelength converter [2]. 
This type of converter has been demonstrated for bit rates up to $10 \mathrm{Gbps}$ [7]. The disadvantages of this type of wavelength conversion are as follows:

- consumes more power than its all-optical counterparts,

- signal is not transparent (signal must be in a specific modulation format and sent at a specific bit rate), and

- information on phase, frequency and amplitude of the optical signal is lost during conversion.

\subsubsection{All - Optical Wavelength Conversion}

All-optical techniques can be further sub-divided into techniques utilizing coherent effects and those using cross modulation.

2.3.2.1 Wavelength Conversion Using Coherent Effects Wavelength conversion using coherent methods is derived from optical responses of a medium when more than one wave is present (wave-mixing). The mixing of multiple waves results in the generation of another wave whose intensity is proportional to the product of the interacting wave intensities. This technique is completely transparent as it preserves the amplitude and phase information of the signal wave. It also provides the opportunity of simultaneous conversion of a set of multiple input wavelengths to another set of multiple output wavelengths. This technique has been demonstrated as potentially reaching bit rates of $100 \mathrm{Gbps}[7]$. 
This technique is detailed in Figure 2-9. In this figure, a value of $n=3$ corresponds to Four-Wave Mixing and a value of $n=2$ corresponds to Difference Frequency Generation. These two techniques are described below.

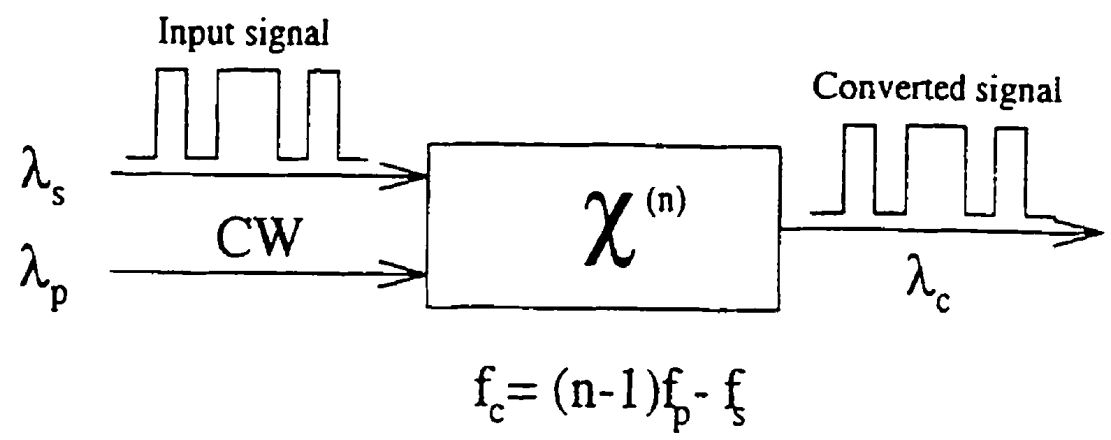

Figure 2-9: A converter based on nonlinear wave-mixing effects [2].

2.3.2.1.1 Four-Wave Mixing (FWM). Four-Wave Mixing $(F W M)$ is a third-order nonlinearity in silica fibres. It is also often referred to as four-photon mixing. This technique causes three optical waves of frequencies $f_{i}, f_{j}$, and $f_{k}(\mathrm{k} \neq \mathrm{i})$ to interact in a multichannel WDM system to generate a fourth wave of frequency given by

$$
f_{i j k}=f_{i}+f_{j}-f_{k}
$$

FWM is a conversion technique that provides modulation-format independence and high bit rate capability [2]. However, it is inefficient with respect to the conversion from pump energy to signal energy. 
Generation $(D F G)$ is a second-order nonlinear interaction of a medium with two optical waves (a pump wave and the signal wave) [7]. DFG does not suffer from the satellite signals that can be present with FWM techniques. It is very fast but still suffers from low efficiency and has a high sensitivity to polarization. The main difficulty with this technique is the phase matching of the interacting waves [2].

2.3.2.2 Wavelength Conversion Using Cross Modulation. Wavelength conversion using cross modulation techniques generally use active semiconductor devices. Semiconductor optical amplifiers (SOAs) and lasers fall into this category. Yoo describes these techniques belonging to a class known as optical-gating wavelength conversion [7]. SOAs can be used for wavelength conversion in one of two modes: cross-gain modulation (XGM) or cross-phase modulation (XPM).

\subsection{SOAs in Cross Gain Modulation (XGM) Mode. In this technique, the} intensity of the input signal is modulated, which in turn modulates the gain in the SOA through gain saturation. This gain variation is used to modulate the output wave (at the desired wavelength, $\lambda_{c}$ ) so that it carries the same information as the original input signal. The wavelength-converted signal is inverted compared to that of the original input signal. Although this type of wavelength conversion is simple, the inversion of the converted wave is a drawback that cannot be ignored. 


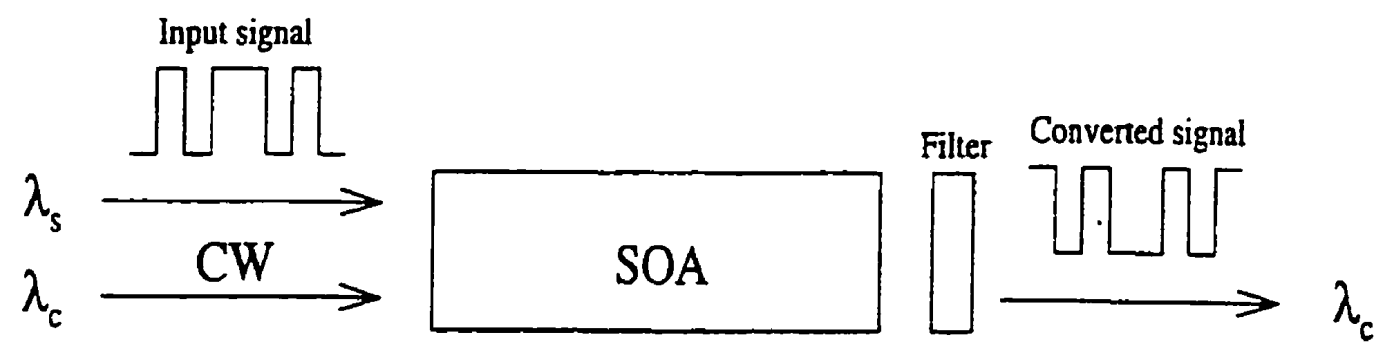

Figure 2-10: A wavelength converter using an SOA in XGM mode [2].

\subsection{SOAs in Cross Phase Modulation (XPM) Mode. By relying on the fact} that the refractive index of an SOA is dependent on the carrier density in its active region, one can create a wavelength converter. By using an incoming signal that depletes the carrier density, the refractive index will be modulated and thereby result in phase modulation of a continuous wave signal (at the desired wavelength, $\lambda_{c}$ ) [2]. By integrating the SOA into an interferometer, an intensity modulated signal format will result at the output of the converter. Figure 2-11 shows an asymmetric Mach-Zender interferometer (MZI) wavelength converter based on an SOA in XPM mode. In this mode, the output signal can be inverted or non-inverted, and has a better power efficiency to that of the XGM mode.
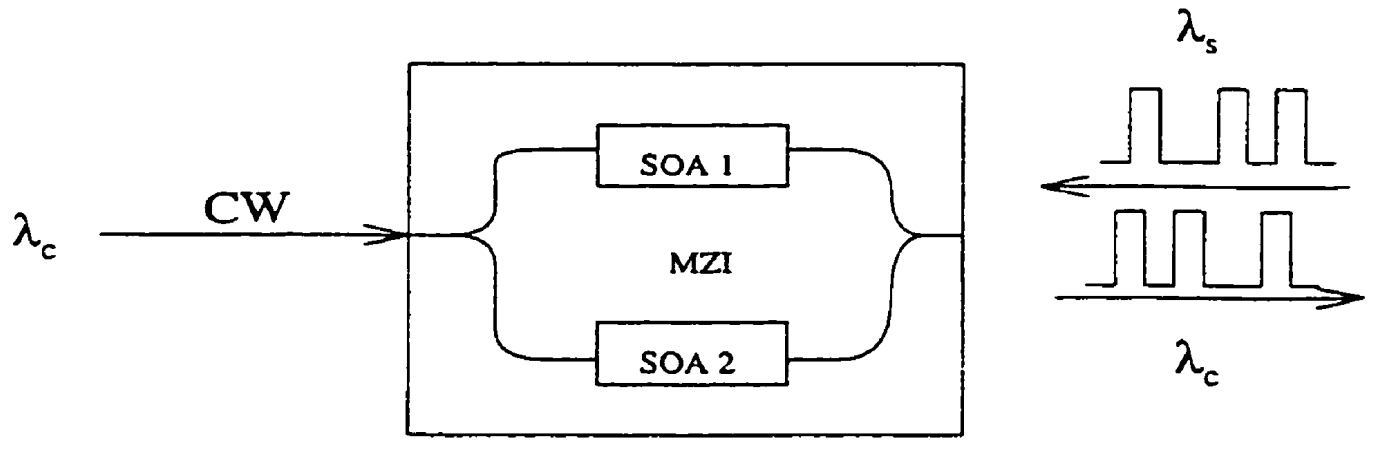

Figure 2-1 1: A wavelength converter using an SOA in XPM mode [2]. 
2.3.2.2.3 Semiconductor Lasers. By using single-mode semiconductor lasers, the lasing mode intensity is modulated by an input signal through lasing mode gain saturation [2]. The input signal saturates the absorption of carrier transitions near the band gap and allows the probe beam to transmit. The output signal (at the appropriate wavelength, $\lambda_{c}$ ) is again inverted compared to the input signal. Due to carrier recombinations, this technique has a bandwidth limit of $1 \mathrm{GHz}[7]$.

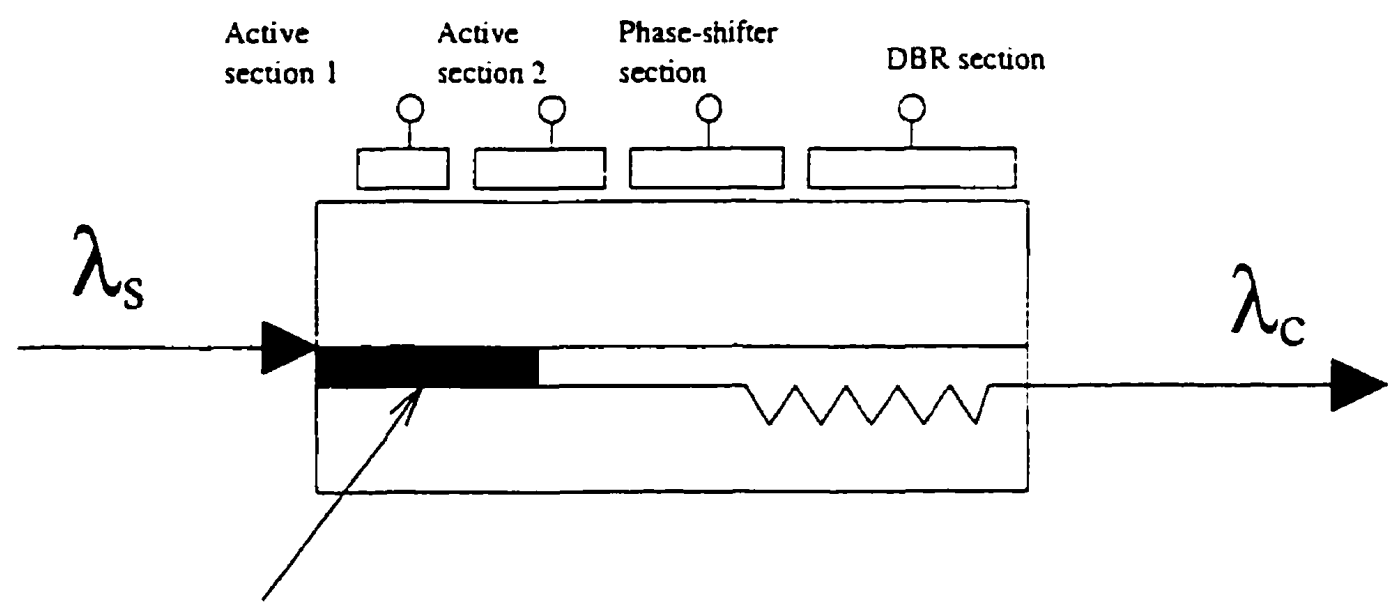

Saturable absorption

Figure 2-12: Conversion using saturable absorption in a laser [2].

There still remains much room for research in the area of wavelength conversion. This section has reviewed some of the various techniques available for designing a wavelength converter. For further reading on wavelength conversion technologies, see $[2],[6]$ and [7]. 


\subsection{Wavelength Convertible Networks}

Wavelength converters are becoming more readily available every day. Many studies have been conducted on the usefulness of wavelength converters in optical networks [3] $[5],[8]-[10]$. Although the studies have used different network topologies (Mesh, ARPA-2, Shufflenet etc), conclusions are similar in all cases: blocking probability decreases and wavelength utilization increases with the use of wavelength converters. The question that now arises is where do we place them in the network? The obvious answer is in the nodes or switches throughout the network.

All optical switches in WDM networks have similar characteristics and information follows a basic flow as it travels through the switch. The incoming fibre optic cables carry multiplexed channels. Therefore, the first step in the optical switch is to demultiplex the channels into their individual wavelengths. The signals then pass through an optical switch, and then get multiplexed with other channels onto an outgoing fibre. Alternatively, after the signals have passed through an optical switch and before getting multiplexed on an outgoing fibre, they may go through an opto-electronic conversion if they will be dropped by the local node, or if it is necessary that they undergo wavelength translation. In this case, they have to pass through another optical switching stage before getting multiplexed on an outgoing fibre. Optical switch architectures differ only by the order of these events, and whether or not resources are shared (i.e. wavelength converters within the switch). 
There are four architectures that are common in optical switch design [2, 5]:

a dedicated wavelength-convertible switch,

a share-per-node wavelength convertible switch,

a share-per-link wavelength convertible switch, and

- an electronic wavelength convertible switch.

This thesis will also introduce a new architecture, the half-clear wavelength convertible switch. The next few sections wil! briefly describe each architecture. A more in depth analysis is presented in Chapter 3.

\subsubsection{Dedicated Wavelength Convertible Switch}

A dedicated wavelength convertible switch is shown in Figure 2-13. This architecture has a dedicated wavelength converter on each outgoing link prior to multiplexing. This architecture is not very efficient, as all of the wavelength converters may not be required simultaneously. This leads to the development of the other switch architectures discussed below.

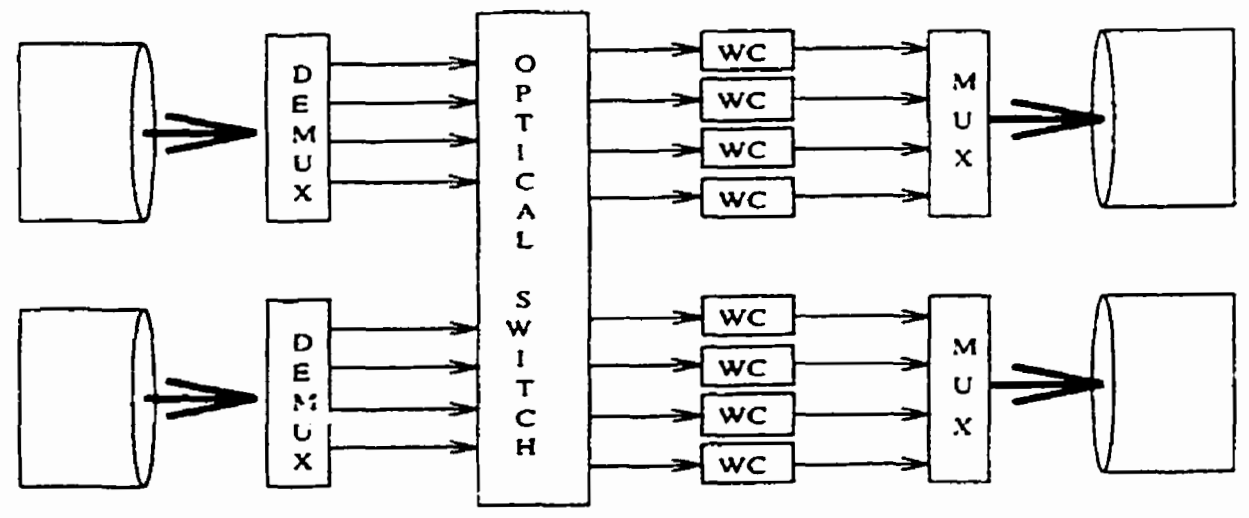

Figure 2-13: A Dedicated Wavelength Convertible Switch [2] 


\subsubsection{Share-Per-Node Wavelength Convertible Switch}

In the share-per-node wavelength convertible switch, the wavelength converters are grouped together in a converter bank and shared between all outgoing links. In this architecture, the input links requiring wavelength conversion are switched to the appropriate port on the first optical switch to the converter bank. After conversion, the signal must travel through another optical switch to be directed to the appropriate output port (fibre). This architecture has the advantage of using a limited number of wavelength converters, but its complexity increases with the addition of a second optical switching stage for those channels requiring conversion.

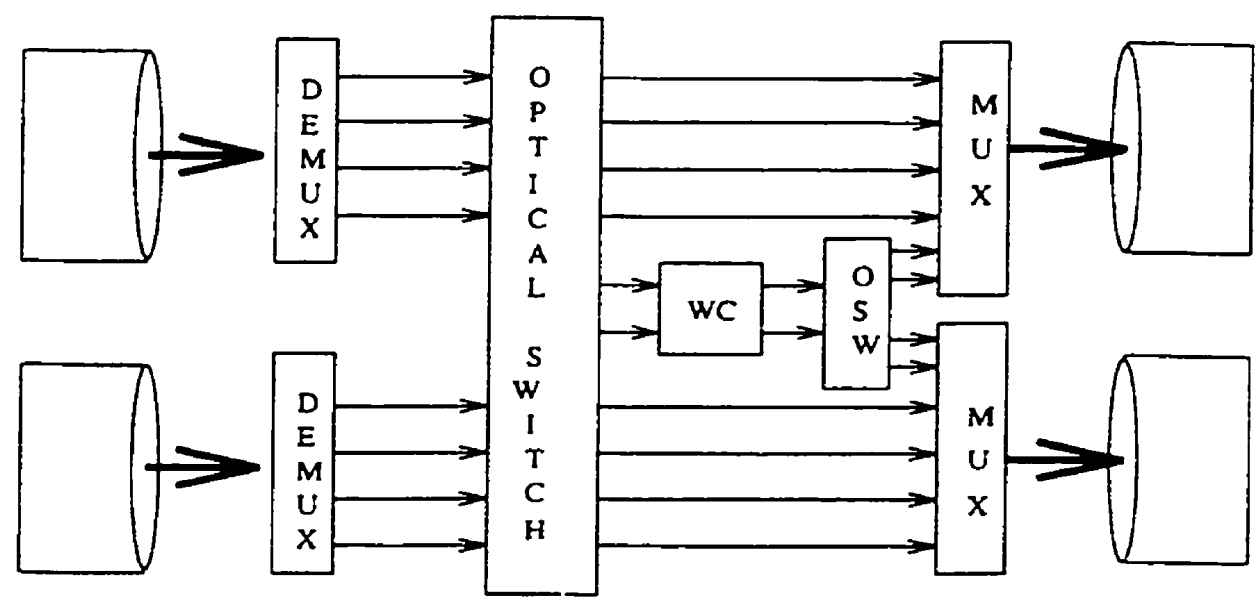

Figure 2-14: A Share-per-Node Wavelength Convertible Switch[2] 


\subsubsection{Share-Per-Link Wavelength Convertible Switch}

In the share-per-link wavelength convertible switch, the wavelength converter banks are found at the outgoing links. Input channels requiring conversion are switched to the appropriate link on a port that passes through the converter bank. This architecture has the advantage over the previous one of not increasing complexity with the addition of another switching stage, while still minimizing the number of converters within the node.

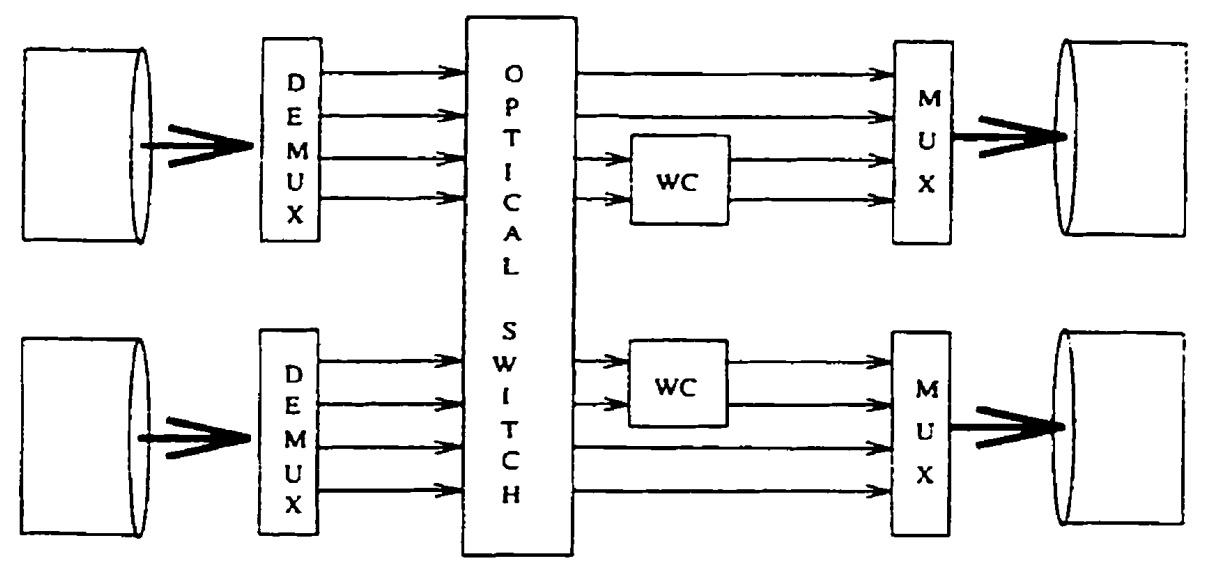

Figure 2-15: A Share-per-Link Wavelength Convertible Switch [2]

Lee and $\mathrm{Li}$ investigate the share-per-node and share-per-link switch architectures as a method of reducing costs by sharing converters within the network nodes [3]. They propose a heuristic algorithm for dynamic routing to reduce the number of converters used in the nodes. Through simulation on an ARPA-2 network, the authors show the performance gain of using wavelength converters in terms of blocking probability and fairness. 


\subsubsection{Electronic Wavelength Convertible Switch}

The final architecture, the electronic wavelength convertible switch, switches channels requiring wavelength conversion to the local network access station where it undergoes opto-electrical conversion. The signal is then retransmitted by the network access station on a new wavelength. This architecture has the advantage of signal regeneration, but is limited in the data rate achievable due to electronic limits.

Kovacevic and Acampora study the benefits of electronic wavelength conversion in a large mesh network as a low cost alternative to all-optical wavelength conversion [4]. They concentrated on the electronic wavelength convertible switch architecture. In this switch, the signal is converted first to the electronic domain, and then converted back to the optical domain on a different wavelength.

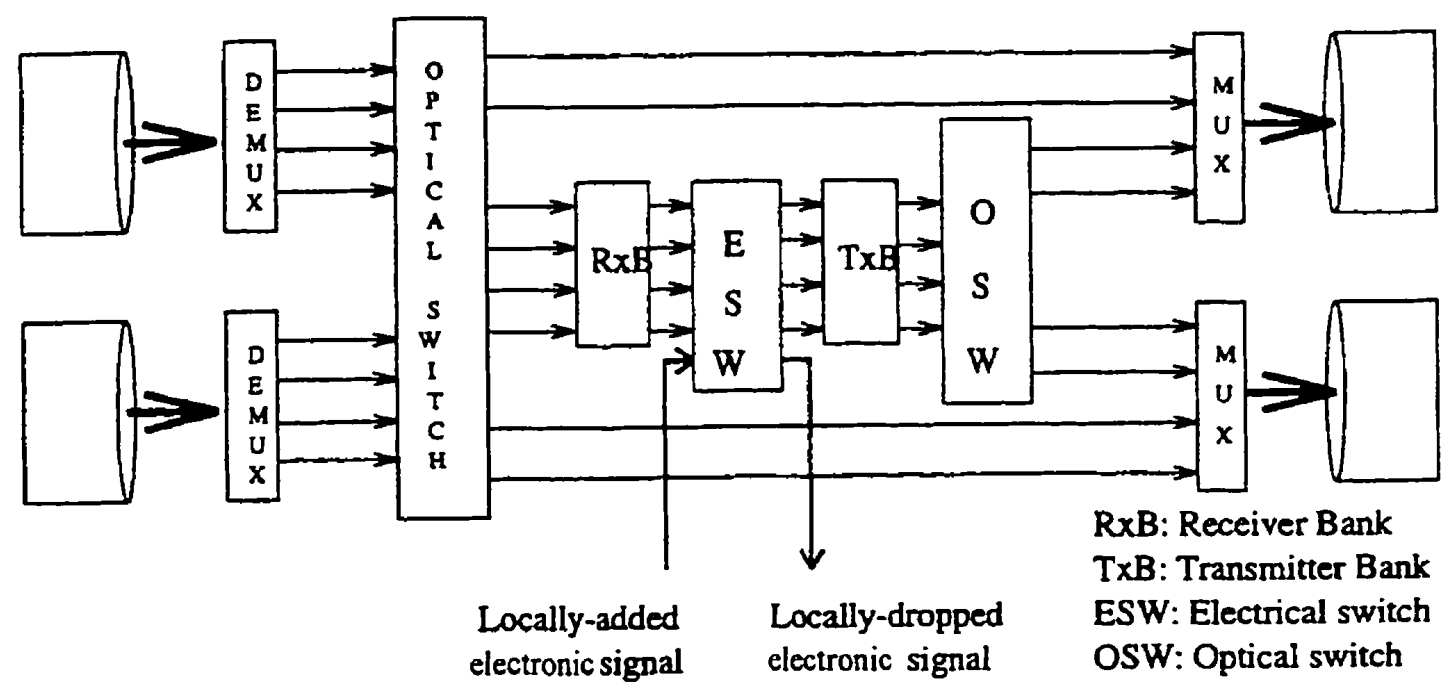

Figure 2-16: An Electronic Wavelength Convertible Switch [2] 
The authors show that with specific wavelength assignment algorithms, they can minimize the number of wavelength conversions necessary. This shows that with such algorithms, electronic conversion can be almost as effective as all-optical techniques. They proposed three wavelength assignment algorithms to achieve this goal.

Each of the three algorithms involves different cost assignments for the links in the network. In Algorithm A, the cost of an edge corresponding to an idle wavelength of an intermediate station is a constant $d_{t}>0$. This minimizes the number of wavelength changes per connection. In Algorithm B, the cost of an edge corresponding to an idle wavelength on an access link of an intermediate station is inversely proportional to the residual capacity of the access link. This avoids electronic conversion on highly utilized stations. In Algorithm C, the cost of an edge corresponding to an idle wavelength on an access link of an intermediate station is equal to a constant $d_{t}>0$ plus a term which is inversely proportional to the residual capacity of the access link. Basically, this is a combination of Algorithm A and Algorithm B. This ensures the number of translations is kept at a minimum. If more than one wavelength assignment results in the same number of translations, then the one with the least loaded access links is chosen.

Using simulation, the authors show that electronic wavelength translation improves the performance of a large mesh network. The difference between all-optical techniques is small and decreases with an increase in network size. As such, the authors indicate that electronic wavelength conversion is a cost effective alternative to all-optical wavelength conversion. 


\subsubsection{Wavelength Convertible Switch Summary}

The following table summarizes the five wavelength convertible switches described in this section. The following abbreviations will be used in the table (and in other figures throughout this thesis):

- DED - dedicated wavelength convertible switch;

- SPN - share-per-node wavelength convertible switch;

- SPL - share-per-link wavelength convertible switch; and

- ELE - electronic wavelength convertible switch.

Table 2-1: Wavelength Convertible Switch Summary

\begin{tabular}{|c|c|c|c|c|}
\hline $\begin{array}{c}\text { \# optical } \\
\text { switching } \\
\text { stages }\end{array}$ & DED & SPN & SPL & ELE \\
\hline $\begin{array}{c}\text { Clear } \\
\text { Conversion? }\end{array}$ & Yes & Yes & Yes & No \\
\hline $\begin{array}{c}\text { Shared } \\
\text { converters? }\end{array}$ & No & Yes & Yes & No \\
\hline $\begin{array}{c}\text { O/E } \\
\text { conversion } \\
\text { required? }\end{array}$ & No & No & No & Yes \\
\hline
\end{tabular}

\subsection{Routing and Wavelength Assignment}

Wavelength Division Multiplexing has already been mentioned as the leading technology in developing all-optical networks. Connecting a source node to a destination node using a WDM channel is the equivalent of adding a new virtual link to the network. Virtual links constitute a virtual topology, which is embedded in the physical topology. It is over the virtual topology that the traffic is routed. 
How to build this virtual topology and assign wavelengths to its links is known as the Routing and Wavelength Assignment (RWA) problem. It has been proven in the literature $[2,11-13]$ that an optimal solution to this problem is NP-hard, since this is an example of a mixed integer linear mathematical problem (MILP), for which no efficient algorithms are known to exist for the solution of arbitrary MILPs. As such, the majority of research in this area has been focused on the development of heuristic approaches to solve the RWA problem.

In a multi-hop wavelength routing network, the RWA problem can be broken down into three sub-problems: first, to choose a virtual topology, second, to assign wavelengths to the virtual links, and third, to route packets over those links. Based on the reconfiguration of the virtual topology and packet switches at the nodes, the RWA problem can be classified into four categories [14]:

- Static-Static RWA: this problem is solved using a traffic matrix that represents the long-term average traffic demands between all node pairs in the network. The solution of this problem is a static virtual topology and a static packet routing scheme. Packeis will follow a predetermined route determined using the average traffic demands. The objectives are to minimize the maximum virtual link utilization and minimize network time delay. Some heuristic techniques have been developed to solve this problem $[2,11,15-16]$ using both regular and irregular network topologies.

- Static-Dynamic RWA: this problem combines the choice of a virtual topology and assigning wavelengths to the virtual links as one sub-problem. This combined subproblem is then solved using a traffic matrix that represents the long-term average 
traffic demands between all node pairs in the network, yielding a static virtual topology. Packets are then routed over the virtual topology dynamically. Some research has been done to heuristically solve this problem [17].

- Adaptive RWA: this problem uses several traffic matrices where each matrix represents the average traffic demand between all node pairs over a certain period of time. The solution yields a static virtual topology and a static routing scheme similar to the static-static RWA problem for each period of time. The network will then adapt its virtual topology and routing scheme based on the problem solution. One objective is to minimize the differences in the virtual topology to avoid disruption when the network is reconfigured. Solutions to this problem have been proposed in $[2,18]$.

- Dynamic RWA: the obvious last step is to reconfigure the virtual topology and the routing scheme dynamically as new connection requests arrive. This is particularly good for connection-oriented networks. An objective of this problem is to minimize the connection request blocking probability. A solution to this problem has been proposed in [19].

For the purpose of this thesis, the static-static RWA problem was used in developing a simulator to assess the performance of wavelength convertible switches. The Wavelength Convertible Optical Network Simulator (WCONSIM) introduced in Chapter 4 was based on the RWA/NCO algorithm introduced in [14], which used the static-static RWA problem as a base. Details of the simulation software is provided in Chapter 4 . 


\subsection{Chapter Summary}

This chapter provided background information relating to this research. It provided an overview of multiplexing techniques, looking specifically at wavelength division multiplexing. Types of WDM networks were discussed with the emphasis placed on wavelength routed networks. Existing technologies for wavelength conversion were presented in Section 2.3. These comprised of opto-electronic conversion techniques as well as all-optical conversion techniques. Wavelength convertible switches were introduced in Section 2.4. Finally, a discussion of the routing and wavelength assignment problem was presented in Section 2.5. The information presented in this chapter will be used $\varepsilon ;$ a base for further discussions and analysis throughout this thesis. 
CHAPTER 3 - THE HALF-CLEAR W A VELENGTH CONVERTIBLE SWITCH AND ITS COMPARISON WITH OTHER ARCHITECTURES

\subsection{Introduction}

Four switch architectures employing wavelength converters are presented in this thesis. They were first introduced in Section 2.4. Each architecture was shown as a block diagram and individual components have not been described. This chapter introduces a novel switch architecture, the half-clear wavelength convertible switch. This chapter also looks into each architecture in more depth and a comparison of the five switch architectures is presented.

\subsection{Half-Clear Wavelength Convertible Switch}

The half-clear wavelength convertible switch is proposed here as an alternative switch architecture. It uses the same approach as the share-per-node switch. The switch has a bank of wavelength converters that are shared with all channels requiring conversion. The difference is that only half of the wavelengths have the ability to be converted at any node in the network. The wavelength switches connected to the converter bank can be configured independently at each node. The remaining half, if conversion is required, are converted by routing through the local access node as in the electronic wavelength convertible switch. 
This arrangement is based on the assumption that no more than half of the wavelengths would ever require conversion at any one switch. By reconfiguring which wavelengths can be converted at each switch, the network should be able to handle any request for traffic routing.

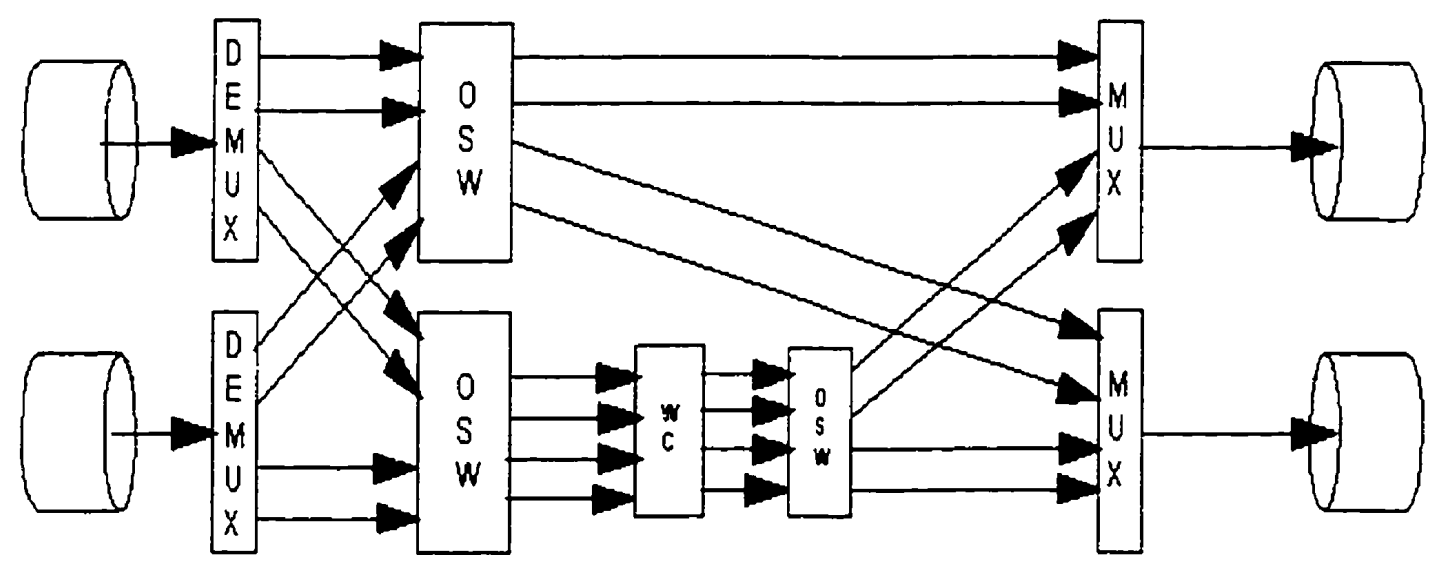

Figure 3-1: A Half-Clear Wavelength Convertible Switch

As an extension to Table $2-1$, the following table compares the previous four switch architectures with the half-clear wavelength convertible switch: 
Table 3-1: Wavelength Convertible Switch Summary

\begin{tabular}{|c|c|c|c|c|c|}
\hline & DED & SPN & SPL & ELE & HALF \\
\hline $\begin{array}{c}\text { \# optical } \\
\text { switching } \\
\text { stages }\end{array}$ & 1 & 2 & 1 & 2 & 1 \\
\hline $\begin{array}{c}\text { Clear } \\
\text { Conversion? }\end{array}$ & Yes & Yes & Yes & No & $\begin{array}{c}\text { Yes/No } \\
\text { (for half of the wavelengths) }\end{array}$ \\
\hline $\begin{array}{c}\text { Shared } \\
\text { converters? }\end{array}$ & No & Yes & Yes & No & $\begin{array}{c}\text { Yes/No } \\
\text { (for half of the wavelengths) }\end{array}$ \\
\hline $\begin{array}{c}\text { O/E } \\
\text { conversion } \\
\text { required? }\end{array}$ & No & No & No & Yes & $\begin{array}{c}\text { Yes/No } \\
\text { (for half of the wavelengths) }\end{array}$ \\
\hline
\end{tabular}

Before a detailed comparison can be completed between the five switch architectures employing wavelength converters, the components that make up the switches must be understood.

\subsection{Switch Components}

The five switch architectures being studied can be constructed from a combination of five core components that are examined in this chapter. They are:

- a 1 2 optical gate (directional coupler),

- a non-blocking optical switch ( $8 \times 8$ or $16 \times 16$ configuration),

- a multi-channel wavelength multiplexer,

- a multi-channel wavelength demultiplexer, and

- a tunable wavelength converter. 
Local access stations, electronic networks and long haul fibre links have been ignored in this comparison. Since they already exist in present networks, they would not add additional cost to any optical network being deployed. Therefore, only the node components themselves are examined.

\subsubsection{1x2 Optical Gates}

The $1 \times 2$ Optical Gate used in this comparison is one of the SW Series of Fibre Optic Switch Modules available from JDS Uniphase [20]. The SW switch connects the optical channel by redirecting the optical signal into a selected output fibre. This is achieved using optical prisms driven by a highly precise mechanism and activated via an electrical control signal. The SW switches are optically passive and are, therefore, transparent to signaling formats [21].

The key features of interest for this evaluation are [21]:

- typical insertion loss of $0.5 \mathrm{~dB}$,

- typical connector insertion loss of $0.2 \mathrm{~dB}$.

- polarization dependent loss of $0.02 \mathrm{~dB}$, and

- typical switching time of $10 \mathrm{~ms}$.

JDS Uniphase quoted the cost of this component as $\$ 300$ US per channel. For a $1 \times 2$ configuration, the cost is $\$ 600$ US. 


\subsubsection{Non-Blocking Optical Switch}

Optical Switches can easily be fabricated using a combination of the $1 \times 2 / 2 \times 1$ optical gates described in the previous section. However, $8 \times 8$ and $16 \times 16$ non-blocking optical switch configurations are available commercially. As this seems to be the more realistic way of acquiring this component, this approach has been used in the evaluations.

Astarté Fibre Networks manufactures the Star*Switch 7200 line of optical switches [22]. The 7200 series switches cross-connect any single input fibre (port) to any single output fibre (port), with a maximum of 72 simultaneous fibre connections [23]. The Star*Switch can be configured in an $8 \times 8$ or $16 \times 16$ configuration, which are the only two of interest for this work.

The characteristics of interest for this evaluation are [23]:

- average connection loss of $1.9 \mathrm{~dB}( \pm 0.5 \mathrm{~dB})$,

- connection time of $150 \mathrm{~ms}$,

- throughput speed of $40 \mathrm{~Gb} / \mathrm{s}$,

- end-to-end photonic connectivity, and

- non-blocking.

Astarté Fibre Networks quoted their $8 \times 8$ switch at $\$ 30,000$ US and their $16 \times 16$ switch at $\$ 40,000$ US. 


\subsubsection{Wavelength Multiplexer / Demultiplexer}

There are numerous WDM multiplexers on the market. For the purpose of this evaluation, the Dense Wavelength Division Multiplexers from ADC/AOFR were used [24]. AOFR's multichannel multiplexing and demultiplexing devices use proved Fused Bionic Taper (FBT) technology combined with Fibre Bragg Gratings (FBG) to give low insertion loss, high wavelength isolation and tight uniformity. AOFR's unique modular DWDM design also provides for upgradeability, allowing wavelengths to be added as needed [25].

The specifications of interest for this evaluation are [25]:

- maximum insertion loss (multiplexer) of $3.3 \mathrm{~dB}$,

- maximum insertion loss (demultiplexer) of $3.8 \mathrm{~dB}$,

- maximum polarization dependent loss of $0.2 \mathrm{~dB}$, and

- typical connector insertion loss of $0.2 \mathrm{~dB}$.

ADC/AOFR has quoted the cost of their multiplexer / demultiplexer components at \$1500 US per channel (or per wavelength). 


\subsubsection{Wavelength Converter}

A thorough search of the Internet and commercial documentation has resulted in only one tunable wavelength converter, and numerous other fixed wavelength converters that are commercially available. The 1901 ICM All-Optical Interferometric wavelength Converter from Alcatel was chosen for this evaluation [26].

The Alcatel 1901 ICM exploits cross-phase modulation in an integrated MachZender (MZ) interferometer based on an all-active MZ-SOA structure. The converter features bit rate transparency, high extinction ratio, small or negative chirp, up and down conversion as well as conversion to the same wavelength [27].

Specifications of interest for this evaluation are [27]:

- typical conrector insertion loss of $0.2 \mathrm{~dB}$, and

- typical operation bit rate of $2.5 \mathrm{Gbps}$.

AIcatel has quoted the cost of their wavelength converter at $\$ 30,000$ US.

\subsubsection{Component Summary}

The following table summarizes the important characteristics for each of the switch components studied in this research. 
Table 3-2 : Summary of Component Characteristics

\begin{tabular}{|l|l|l|l|l|l|}
\hline Component & $\begin{array}{l}\text { Cost } \\
\text { (US \$) }\end{array}$ & $\begin{array}{l}\text { Processing } \\
\text { Speed } \\
\text { (Gbps) }\end{array}$ & $\begin{array}{l}\text { Losses } \\
\text { (dB) }\end{array}$ & Source & Notes \\
\hline $\begin{array}{l}\text { Ix2/2x1 Optical } \\
\text { Switch }\end{array}$ & 600 & 10 & 0.52 & {$[20-21]$} & \\
\hline 8x8 Optical Switch & 30000 & 40 & 1.90 & {$[22-23]$} & \\
\hline $\begin{array}{l}\text { 16x16 Optical } \\
\text { Switch }\end{array}$ & 40000 & 40 & 1.90 & {$[22-23]$} & \\
\hline $\begin{array}{l}\text { Wavelength } \\
\text { Multiplexer }\end{array}$ & $1500^{*}$ & 2.5 & 3.50 & {$[24-25]$} & *per channel \\
\hline $\begin{array}{l}\text { Wavelength De- } \\
\text { Multiplexer }\end{array}$ & $1500^{*}$ & 2.5 & 4.00 & {$[24-25]$} & *per channel \\
\hline $\begin{array}{l}\text { Wavelength } \\
\text { Converter }\end{array}$ & 30000 & 2.5 & $0.00^{*}$ & {$[26-27]$} & $\begin{array}{l}\text { *optical } \\
\text { regeneration }\end{array}$ \\
\hline
\end{tabular}

\subsubsection{Other Important Measures}

In addition to the characteristics described in the previous sections, there are other important measures used in this research that require their own mention.

3.2.6.1 Packet Size. The choice of a packet size for network traffic can be arbitrary. IP packets do not have a fixed size and as such, can sometimes be difficult to simulate. This work assumes a fixed packet size of 53 bytes (same as a typical ATM packet). Should traditional IP traffic be used through the optical networks studied, it is assumed that another protocol layer would be added to break the traditional IP packet up into 53 byte chunks at the transmitting node, and then combine the chunks into the original IP packet at the receiving node. This will add extra overhead that will be included in the 53-byte packet length. The extra time for an additional protocol layer has been ignored.

3.2.6.2 Time Unit. The choice of a time unit for simulation can also be an arbitrary decision. For ease of computation, 1 ns was used throughout the calculations. This 
corresponds to a processing time of 11 time units per packet for equipment running at 40 Gbps, 43 time units per packet for equipment running at $10 \mathrm{Gbps}$, and 170 time units per packet for equipment running at $2.5 \mathrm{Gbps}$.

3.2.6.3 Switching Delay. Since optical switches still rely on electronic circuitry for control mechanisms, there is inherent in each switch some delay to process the control signal. This switching delay varies depending on the switch in question. This delay is separate from processing time (time it takes the packet to pass through the component) and is usually unknown. To overcome this uncertainty, a buffer of $5 \mathrm{~ms}$ for a $1 \times 2$ optical switching element, and $10 \mathrm{~ms}$ for an $8 \times 8$ or $16 \times 16$ switch has been added to the processing time for these components. Therefore, the total switching delay for a $1 \times 2$ optical switching element would be $15 \mathrm{~ms}(10 \mathrm{~ms}+5 \mathrm{~ms})$ [21]. The total switch delay for an $8 \times 8$ or $16 \times 16$ optical switch would be $160 \mathrm{~ms}(150 \mathrm{~ms}+10 \mathrm{~ms})$ [23].

\subsection{Node Architecture}

A WDM-based network that supports packet switching traditionally consists of a group of nodes connected via fibre optic links. Each network node can be accessed by other network components or terminating equipment such as end users, subnets or packet switches. Two node architectures were investigated for this research: single-fibre architecture and multiple-fibre architecture. These architectures are shown in Figure 3-2 and Figure 3-3 respectively. Each type consists of three parts: the optical switching matrix, the interface and the electronic switching matrix. 

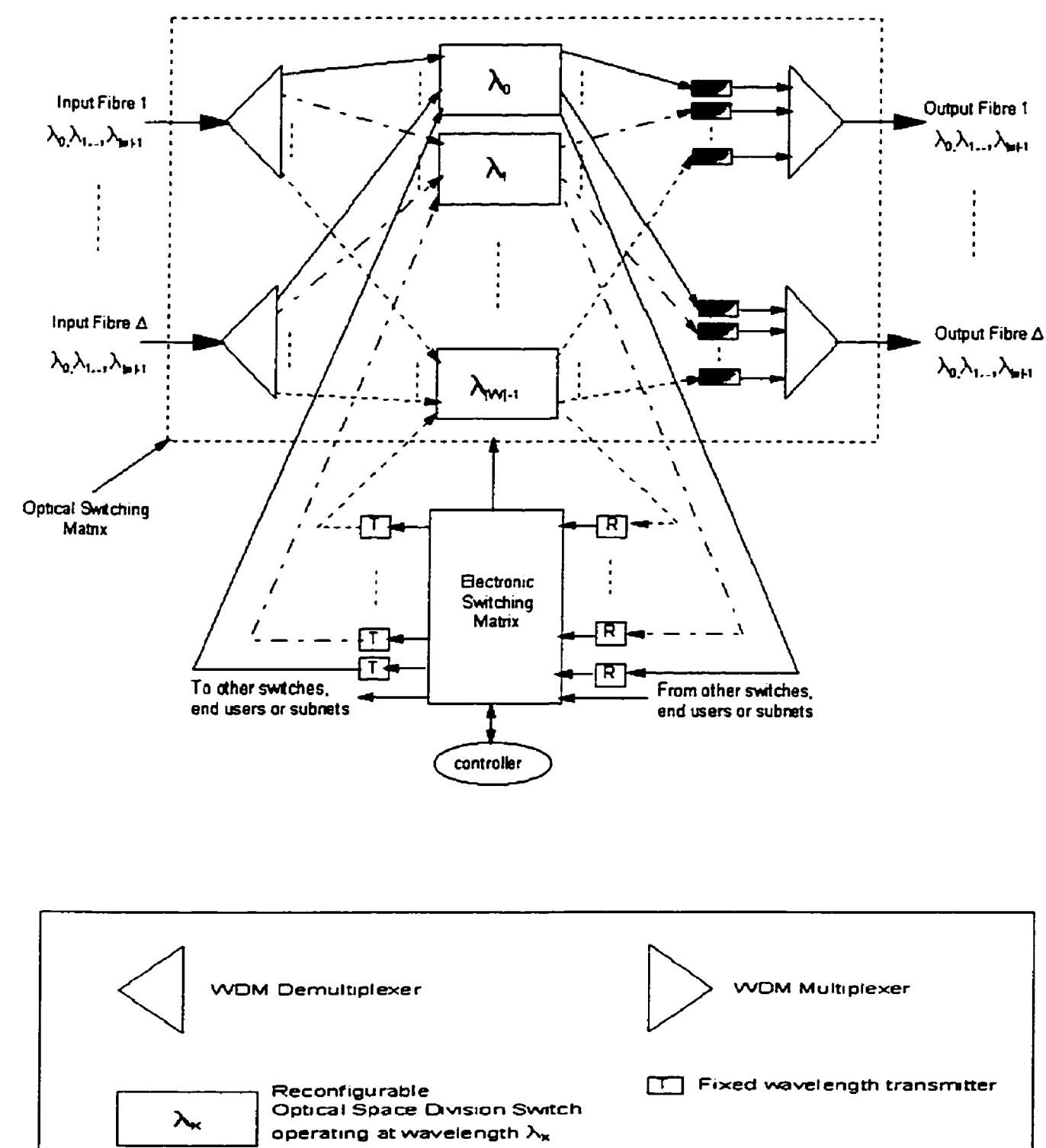

Reconfigurable Optical Space Divsion Switen operating at wavelength $\lambda_{k}$

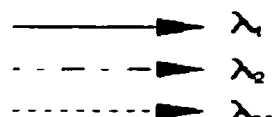

TT Fixed wavel ength transmutter

[R Fixed wavelength recener

Figure 3-2: Single Fibre Architecture 


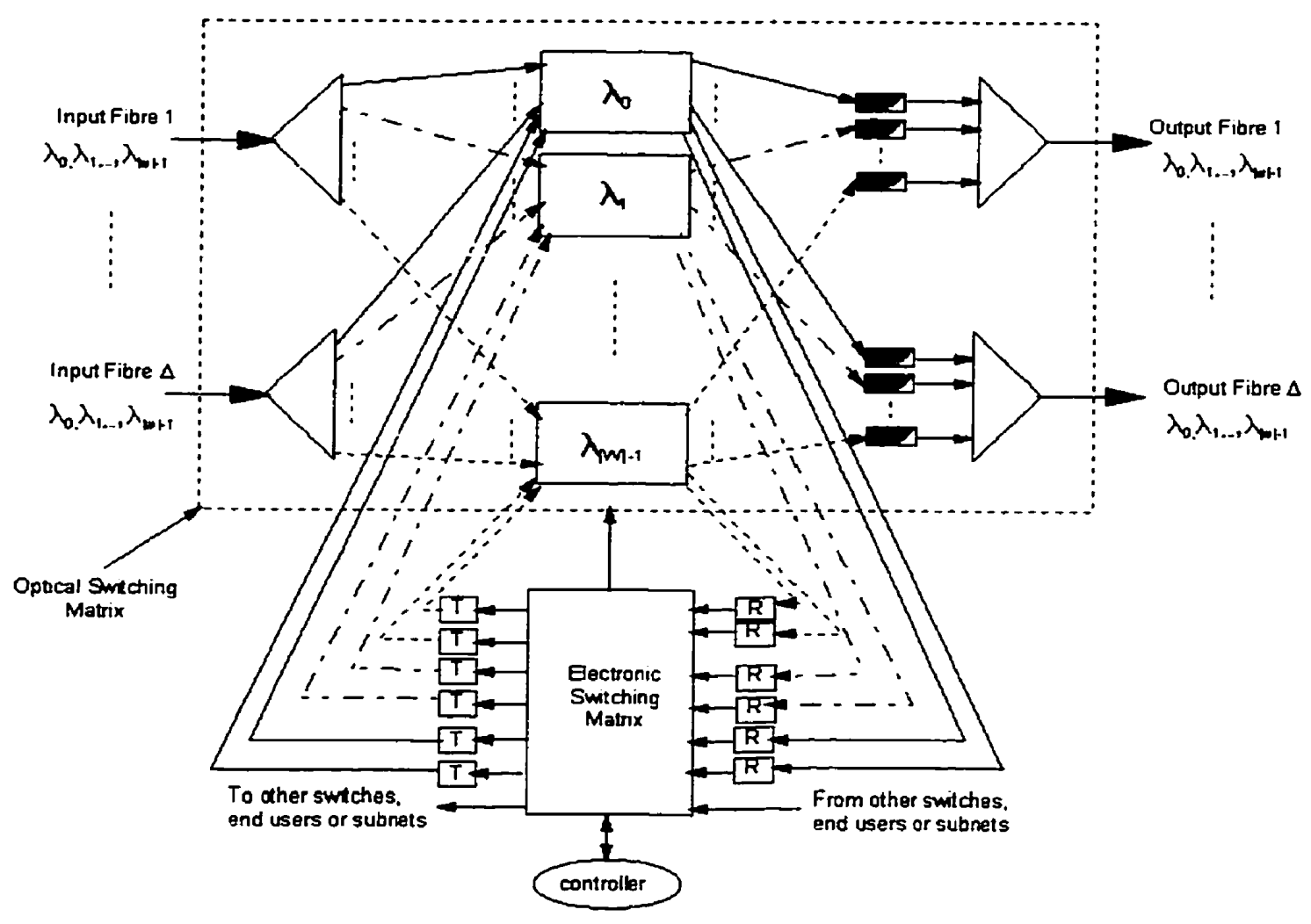

$\int$ wom Demultiplexer
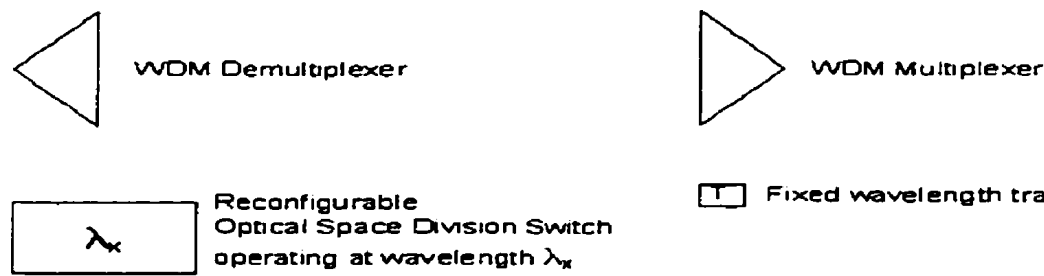

Reconfigurable

Optical Space Division Switch

operating at wavelength $\lambda_{x}$

T] Fixed wavelength transmitter

$\longrightarrow \lambda_{1}$

$\cdots \cdots-\lambda_{2}$

[2] Fixed wavelength receiver

Wavelength Converter

Figure 3-3: Multiple Fibre Architecture 


\subsubsection{The Optical Switching Matrix}

The optical switching matrix consists of four stages: an array of WDM demultiplexers, optical space-division switches, wavelength converters in a particular setup depending on the switch in question, and finally an array of WDM multiplexers. There are $\Delta_{p}$ demultiplexers and multiplexers in each array, where $\Delta_{p}$ represents the physical degree of the node.

Each demultiplexer separates the wavelengths arriving on an input fibre link and directs each wavelength to a different output port. Each input fibre can carry up to $|W|$ wavelengths, where $|W|$ is the cardinality of set $W$, which is the set of all wavelengths in the network. Each multiplexer obviously performs the opposite function.

The optical switching matrix also consists of an array of optical space-division switches. Each array consists of $|W|$ optical switches each operating at a different wavelength. That is, all inputs to a particular optical space-division switch are at wavelength $\lambda_{k}$, where $k \in\{0,1, \ldots, i W-1\}$. The outputs of the space-division switches are directed to a different multiplexer or wavelength converter bank depending on the switch architecture in question.

Each optical switch can route an incoming signal directly from the demultiplexer to a multiplexer bypassing the local node, or it can route the signal to the interface part for further processing by the local node. It is important to have a non-blocking switch in order to route any input port to any output port. 
If the signal at a wavelength $\lambda_{k}$ is directed to an output fibre where another signal at $\lambda_{k}$ is already present, wavelength conversion is required (or the signal is blocked if no conversion is possible). Depending on the switch architecture in use, the signal will pass through a dedicated wavelength converter (dedicated wavelength convertible switch), be routed to a bank of wavelength converters (share-per-node, share-per-link or half-clear wavelength convertible switch), or it can be routed to the local node for conversion (electronic wavelength convertible switch).

It is conceivable to avoid the use of optical space-division switches when considering a static wavelength-routed network. However, since network survivability (link failure) and reconfigurability (traffic pattern changes) are important considerations, the switches have been included in this evaluation.

\subsubsection{Interfacing Part}

The interfacing part performs the task of opto-electronic conversion. It consists of an array of fixed wavelength receivers, and an array of fixed wavelength transmitters. The transmitters convert the signal coming from the electronic switching matrix to a particular wavelength. Conversely, the receivers convert an optical signal from the optical switching matrix to an electronic signal.

The only difference between the single fibre architecture and the multiple fibre architecture is how the wavelengths are assigned to the transmitters and receivers. In the single fibre architecture, each transmitter and receiver must operate on a unique wavelength. Therefore, each optical switch operating at a particular wavelength has a 
single input and a single output connected to the interfacing part. The number of transmitters and receivers are bounded by the following equality:

$$
\left|T_{i}\right| \leq|W| \text { and }\left|R_{i}\right| \leq|W|
$$

where $\left|T_{i}\right|$ and $\left|R_{i}\right|$ indicate the number of transmitters and receivers at Node $i$ and $|W|$ is the number of wavelengths.

In the multiple fibre architecture, the restriction of using a unique wavelength for each transmitter and receiver is lifted. A group of transmitters and receivers can share a particular wavelength as long as each processes a signal arriving from or destined to a different adjacent network node. Therefore, any optical switch can have multiple inputs and outputs connected to the interfacing part. The number of transmitters and receivers is bounded by the following equality:

$$
\left|T_{i}\right| \leq|W| \cdot \Delta_{p}^{i} \quad \text { and } \quad\left|R_{i}\right| \leq|W| \cdot \Delta_{p}^{i}
$$

where $\left|T_{i}\right|$ and $\left|R_{i}\right|$ are the number of transmitters and receivers at node $i, \Delta_{\mathrm{p}}^{\mathrm{i}}$ is the physical degree of node $i$ and $|W|$ is the number of wavelengths.

By comparing equation (3-1) and (3-2), it is obvious that there is a greater spatialreuse of wavelengths when the multiple fibre architecture is used. This results in fewer wavelengths required per fibre. In our comparisons later in this chapter, the optical switches used have enough vacant ports (after the connections to the demultiplexers, multiplexers and converter banks are made) to take advantage of the multiple fibre architecture. As such, the multiple fibre architecture has been used in our evaluations throughout this thesis. 


\subsubsection{Electronic Switching Matrix}

The electronic switching matrix can be any type of electronic switching network (such as an IP router or ATM switch). Inputs and outputs are connected to the optical switching matrix through the interfacing part. Other inputs and outputs can be connected to end users, other networks or sub-networks or other electronic switching devices. The electronic switching matrix is important when attempting to maximize resource utilization through the use of unclear lightpaths. This refers to the use of a sequence of clear lightpaths connected through intermediate nodes. The packets are converted to the electronic domain at the intermediate nodes, and then retransmitted on the new clear lightpath. If the wavelength is different between these two lightpaths, electronic wavelength conversion is done at the intermediate node.

The costs associated with the intermediate node have been ignored for the purpose of the deterministic comparison in this chapter. It is assumed that the local nodes exist in today's network so no additional costs would be incurred. The local node comes into play in the simulation study discussed in Chapters 4 and 5 .

\subsection{Comparison of Wavelength Convertible Switches}

The following sub-sections detail the individual wavelength convertible switches studied through the course of this research. For each of the following switches, the following assumptions were made: 
- For initial costing, 16 wavelengths were used. Costs to increase or decrease the number of wavelengths will be summarized at the end.

- It is assumed that the node has the same number of input fibres as output fibres. For initial costing, a physical degree of four was used for the nodes. Costs have been calculated for a physical degree of one to four and are presented at the end.

- Multiple fibre architecture has been used throughout the calculations as indicated in section 3.2.2. In some cases it was necessary to increase the optical switch sizes to $16 \times 16$ in order to keep the comparison uniform.

- Fibre patch cords connecting the interface part with the optical switch matrix are assumed to have a negligible propagation delay due to the short distances involved. As such, propagation delay has been ignored.

- Optical concentrators used in the share-per-link wavelength convertible switch, and second stage optical switching in the share-per-node and halfclear wavelength convertible switches are constructed using $1 \times 2$ optical gates.

\subsubsection{The Dedicated Wavelength Convertible Switch}

A detailed diagram of the dedicated wavelength convertible switch can be found in Figure 3-4. This architecture uses $8 \times 8$ optical switches, and each output port is equipped with a wavelength converter. 


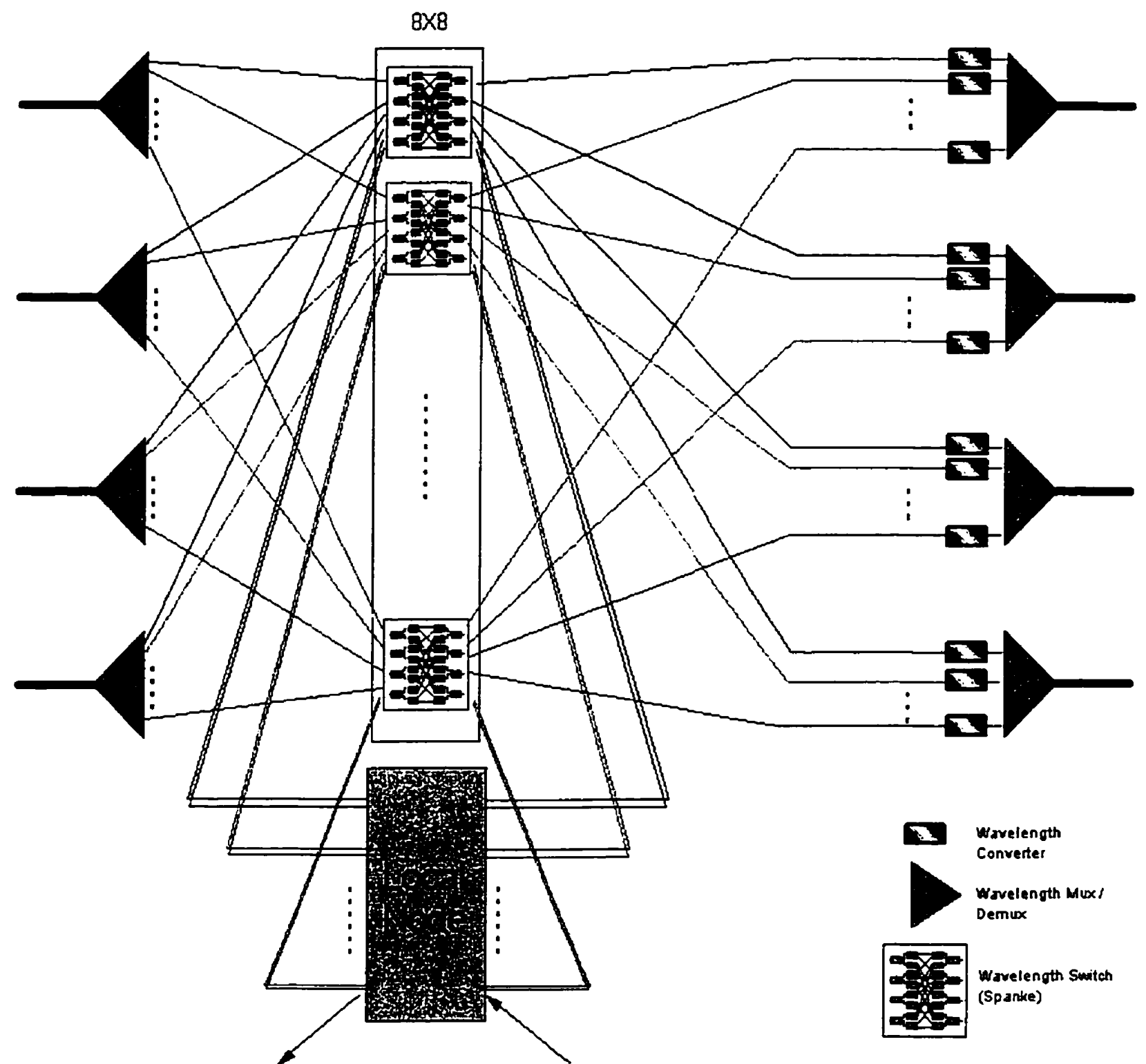

Locally added / dropped traffic to electronic network

Figure 3-4: Detail of a Dedicated Wavelength Convertible Switch 


\subsubsection{The Share-Per-Node Wavelength Convertible Switch}

A detailed diagram of the share-per-node wavelength convertible switch can be found in Figure 3-5. Each optical switch in this architecture has an output connecting to each multiplexer directly, one output connecting to the converter bank, and four outputs connecting to the interfacing part. This results in the need for a $16 \times 16$ optical switch configuration.

There have been no studies done on the optimum number of wavelength converters to have in the converter bank. For the purpose of this analysis, it is assumed that each wavelength has one converter in the bank. Therefore, the number of converters in the converter bank for this architecture is assumed to be $|W|$

The second stage of optical switch required after the converter bank consists of $|W| 1 \times 4$ optical switches. These are constructed from $31 \times 2$ optical switch elements, and are used to switch the signal coming from the wavelength converter to the appropriate output fibre.

\subsubsection{The Share-Per-Link Wavelength Convertible Switch}

A detailed diagram of the share-per-link wavelength convertible switch can be found in Figure 3-6. Each optical switch has two outputs directed to each output fibre. One is connected directly to the multiplexer, while the other is connected to a converter bank associated with that particular output fibre. Another four outputs are connected to the interface part. This results in the need for a $16 \times 16-$ switch configuration. 


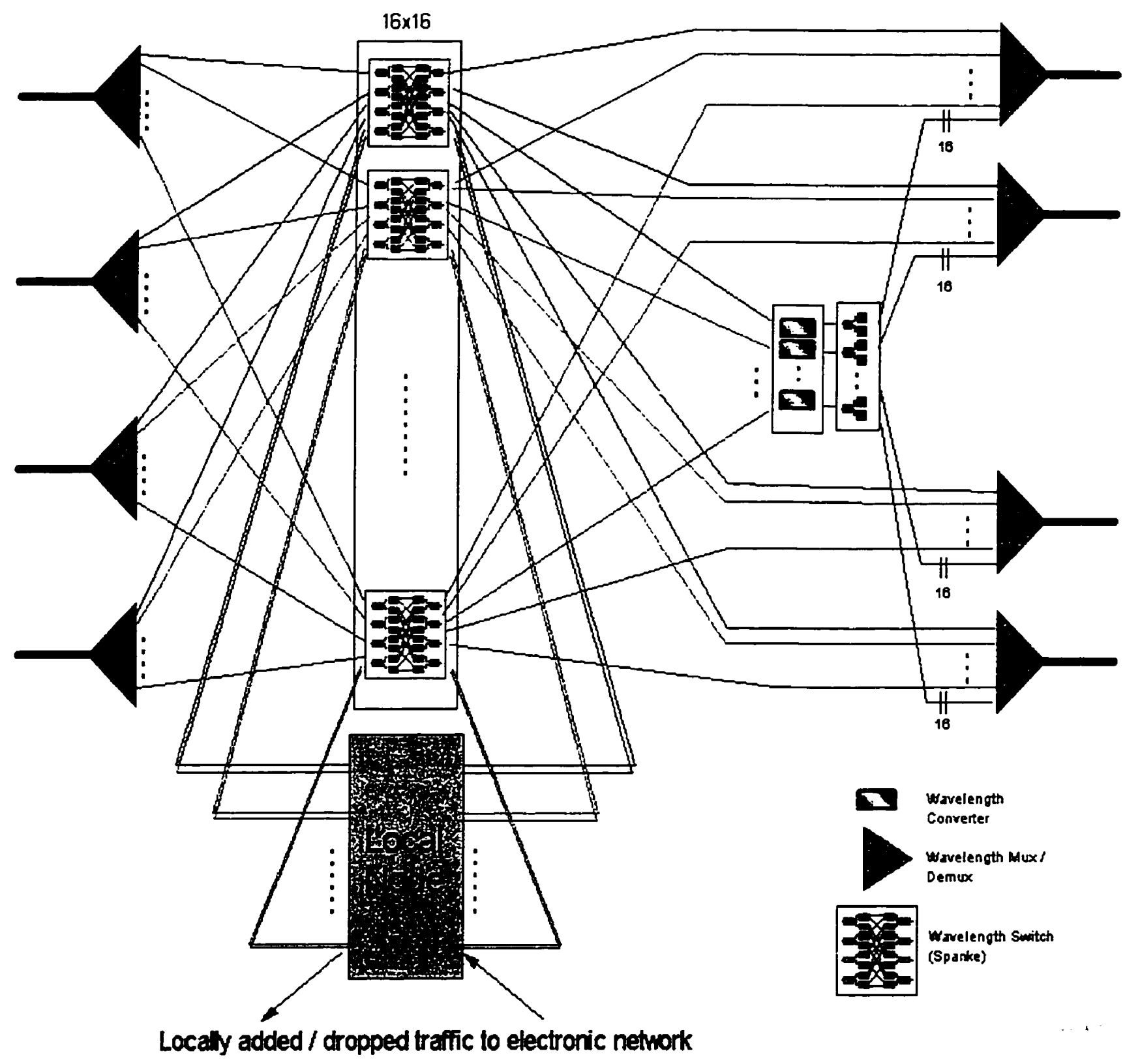

Figure 3-5: Detail of a Share-Per-Node Wavelength Convertible Switch 


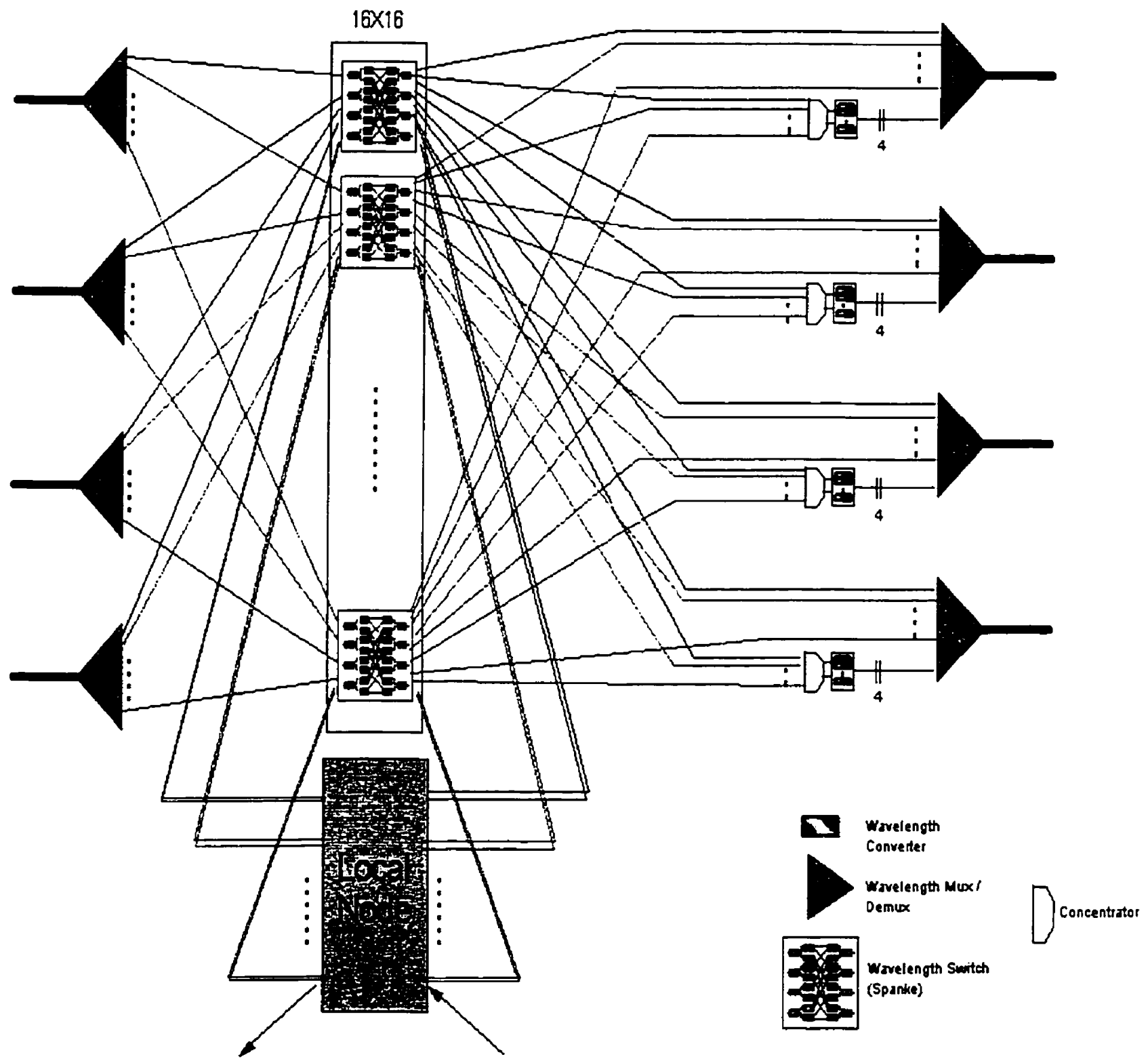

Locally added / dropped trafic to electronic network

Figure 3-6: Detail of the Share-Per-Link Wavelength Convertible Switch 
For the sake of this comparison, the number of wavelength converters in each converter bank is assumed to be $|W| \Delta_{p}$, in the case shown in Figure 3-6, that equates to four converters in each converter bank. A 16x4 optical concentrator is required before the converter bank to connect the switches with the four available converters. Control mechanisms are assumed to be in place within the node to avoid a conflict at the converter. If a converter is not available, the connection is blocked. The $16 \times 4$ optical concentrator is composed of $122 \times 1$ optical switches.

\subsubsection{The Electronic Wavelength Convertible Switch}

A detailed diagram of the electronic wavelength convertible switch can be found in Figure 3-7. This is the simplest of the switch configurations. There are no wavelength converters to be concerned about. $8 \times 8$ optical switches are used with four outputs connected directly to the multiplexers, and the remaining four connected to the interfacing part.

\subsubsection{The Half-Clear Wavelength Convertible Switch}

A detailed diagram of the half-clear wavelength convertible switch is shown in Figure 3-8. This architecture is similar to that of the share-per-node architecture. In this setup, half of the wavelengths are connected to a wavelength converter bank (similar to the share-per-node), and half have no connection to the convert bank (similar to optoelectronic). This means that there is a requirement for both $8 \times 8$ and $16 \times 16$ switch configurations. 


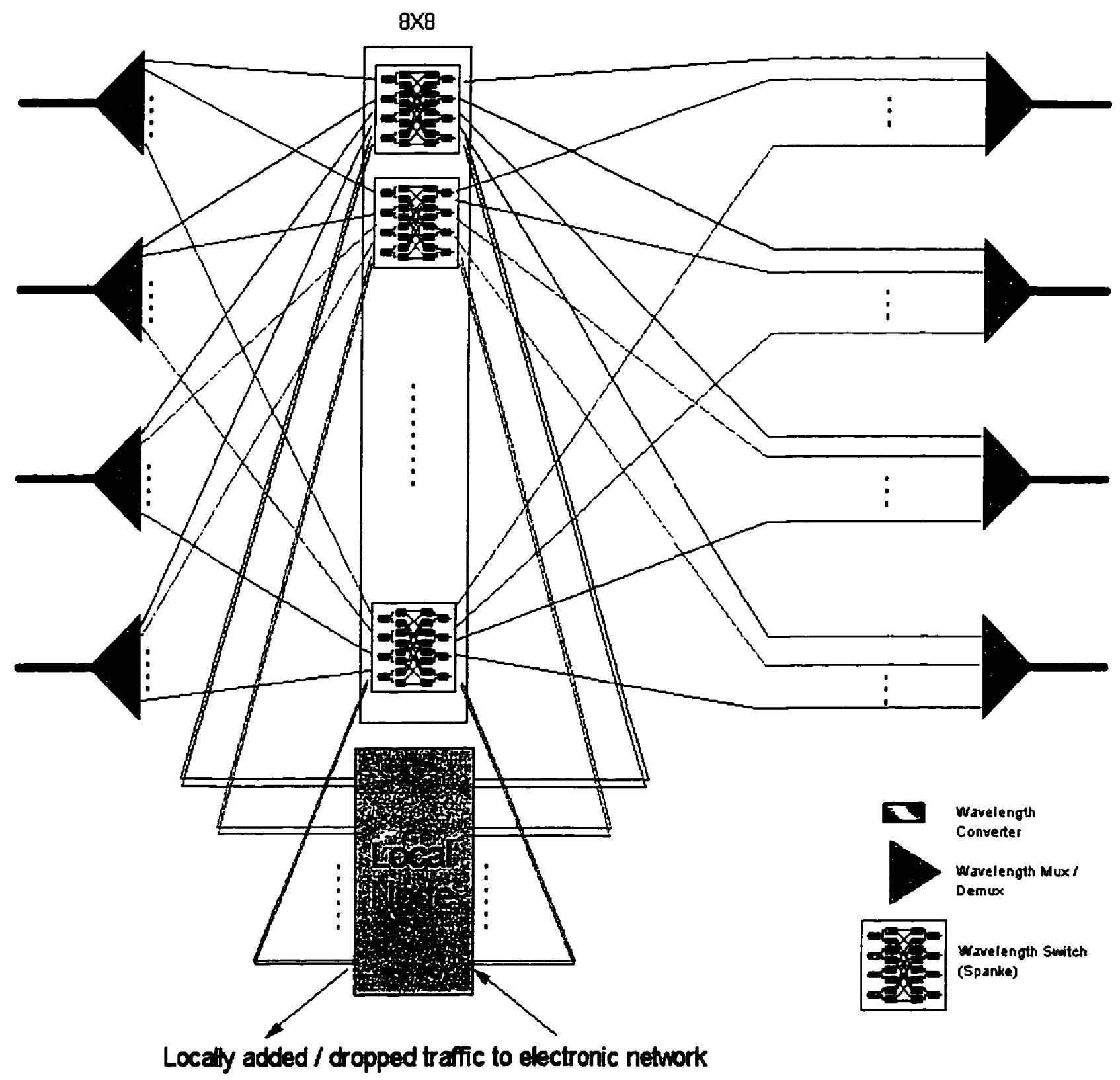

Figure 3-7: Detail of the Electronic Wavelength Convertible Switch 


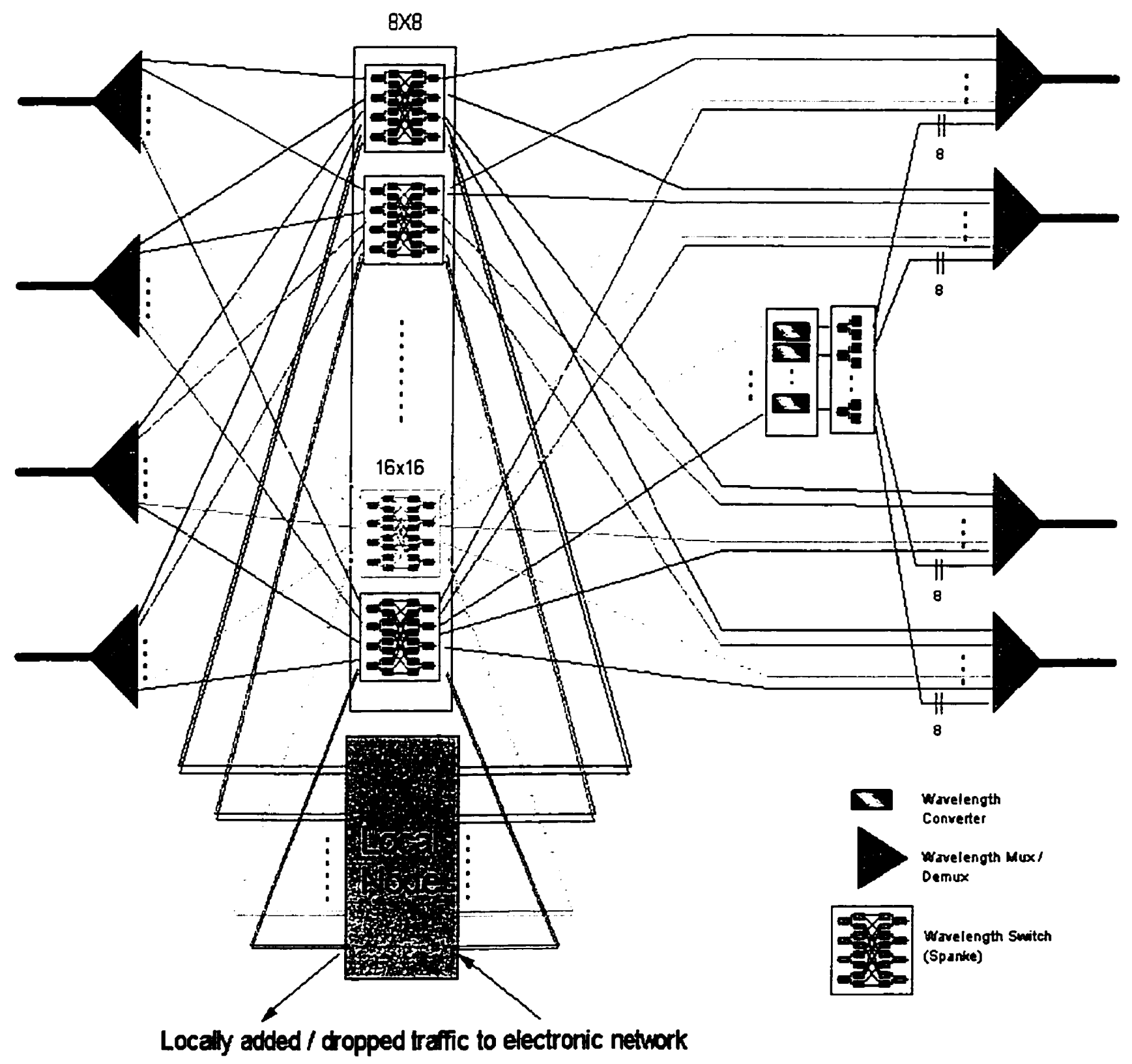

Figure 3-8: Detail of the Half-Clear Wavelength Convertible Switch 
The number of converters in the converter bank is assumed to be $\mid W / 2$ since only half of the total number of wavelengths use the converter bank. The second stage of optical switch required after the converter bank consists of $\mid W / 21 \times 4$ optical switches. These are constructed from $31 \times 2$ optical switch elements, and are used to switch the signal coming from the wavelength converter to the appropriate output fibre.

\subsubsection{Results of Analysis}

The five switches discussed in the previous sections were evaluated against each other by considering the following aspects:

- component costs, including demultiplexers, optical switches, fibre patch cords, wavelength converters and multiplexers, with $|W|=16$ and $\Delta_{p}=4$. (Figure 3-9)

- incremental costs associated with changing the physical degree of the node. Costs were calculated for a physical degree of one to four with $|W|=16$. (Figure 3-10)

- incremental costs associated with changing the number of wavelengths in the system with $\Delta_{p}=4$. (Figure 3-11)

- attenuation (in $\mathrm{dB}$ ) of signal as it passes through the node. This measurement is important when considering placement of optical amplifiers. As this is beyond the scope of this thesis, only the $\mathrm{dB}$ loss calculation has been completed. (Figure 3-12)

- processing time for the packet to pass through the switch. This is measured 
by processing speeds and set up times of the individual components for a packet size of 424 bits. Queuing delay and processing speed for the electronic switch matrix has been estimated at $200 \mathrm{~ns}$. This relates to a processing speed of $2.5 \mathrm{~Gb} / \mathrm{s}$ and a buffer of 30ns for queuing delay. (Figure 3-13)

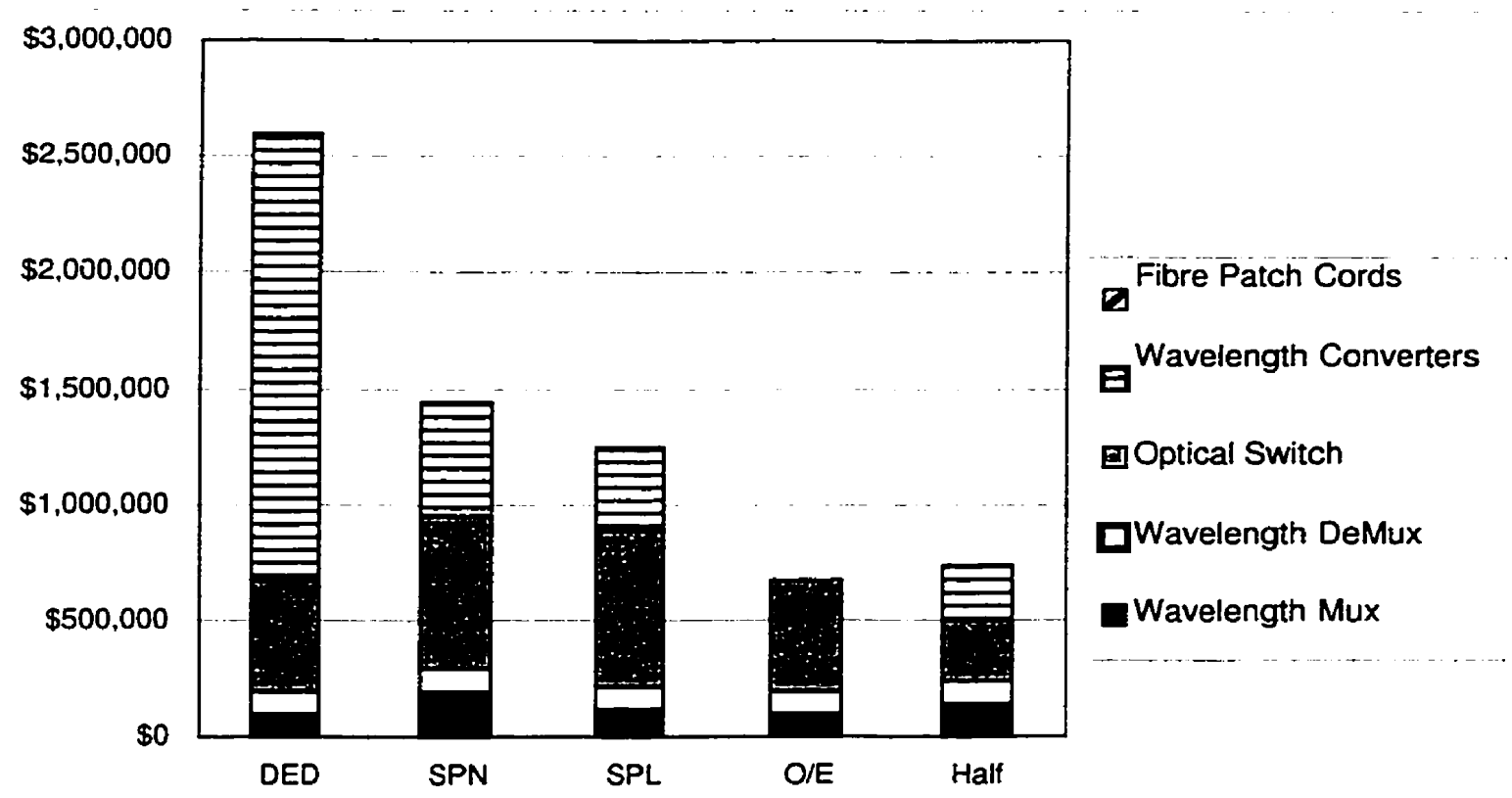

Figure 3-9: Component Costs of Optical Switches, $\Delta_{p}=4,|W|=16$ 


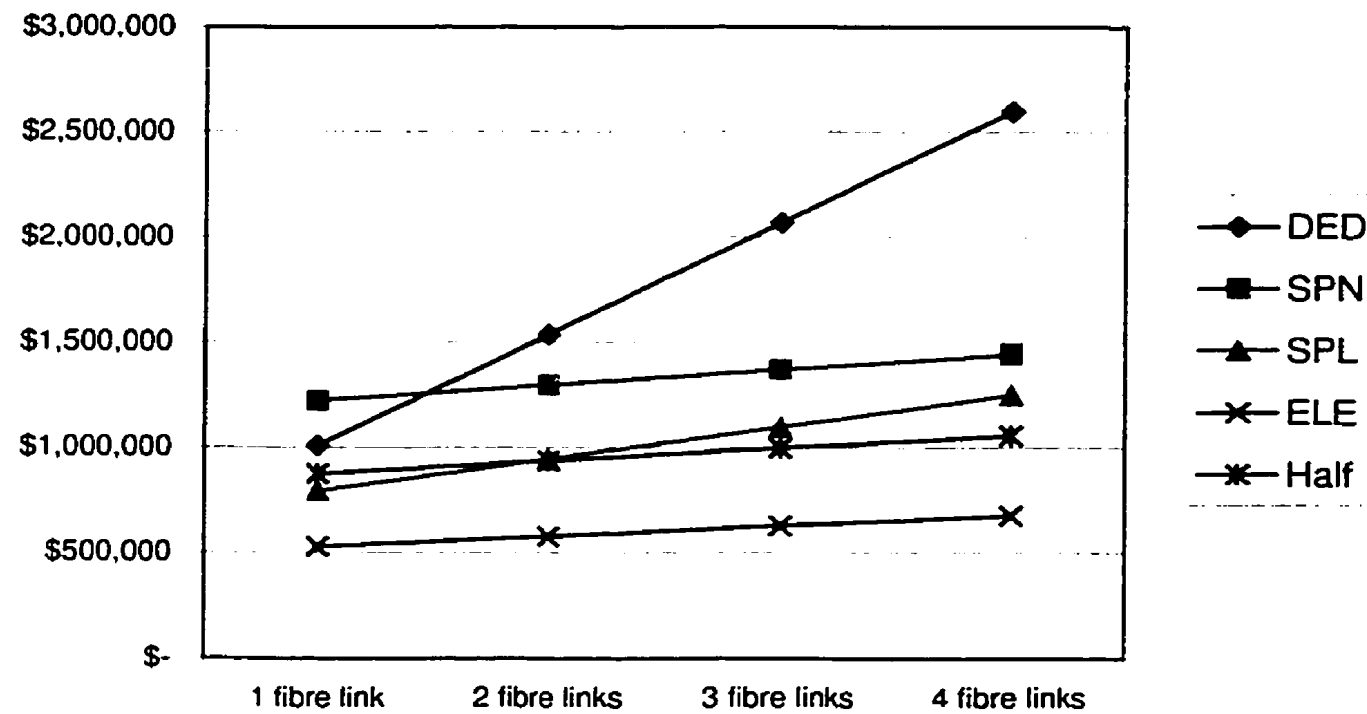

Figure 3-10: Incremental Costs associated with $\Delta_{p},|W|=16$.

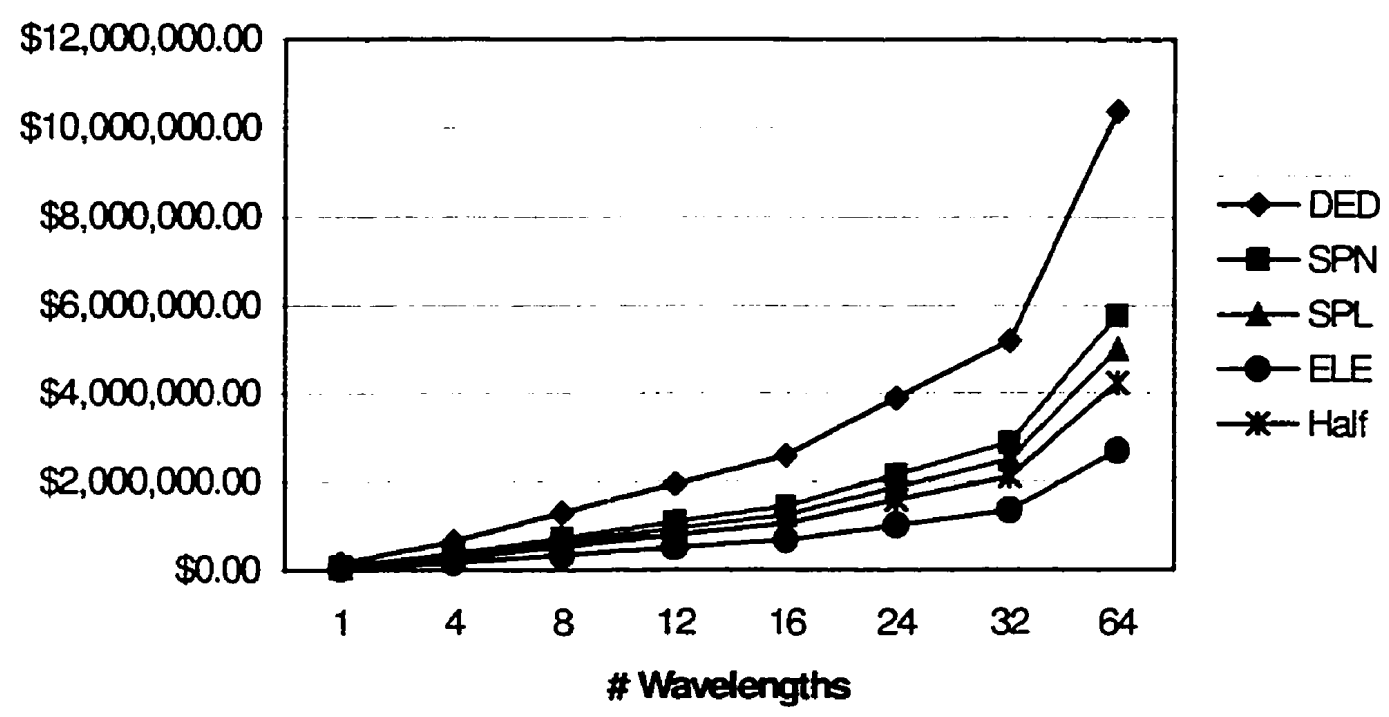

Figure 3-1 1: Incremental Costs associated with $|W|, \Delta_{p}=4$ 


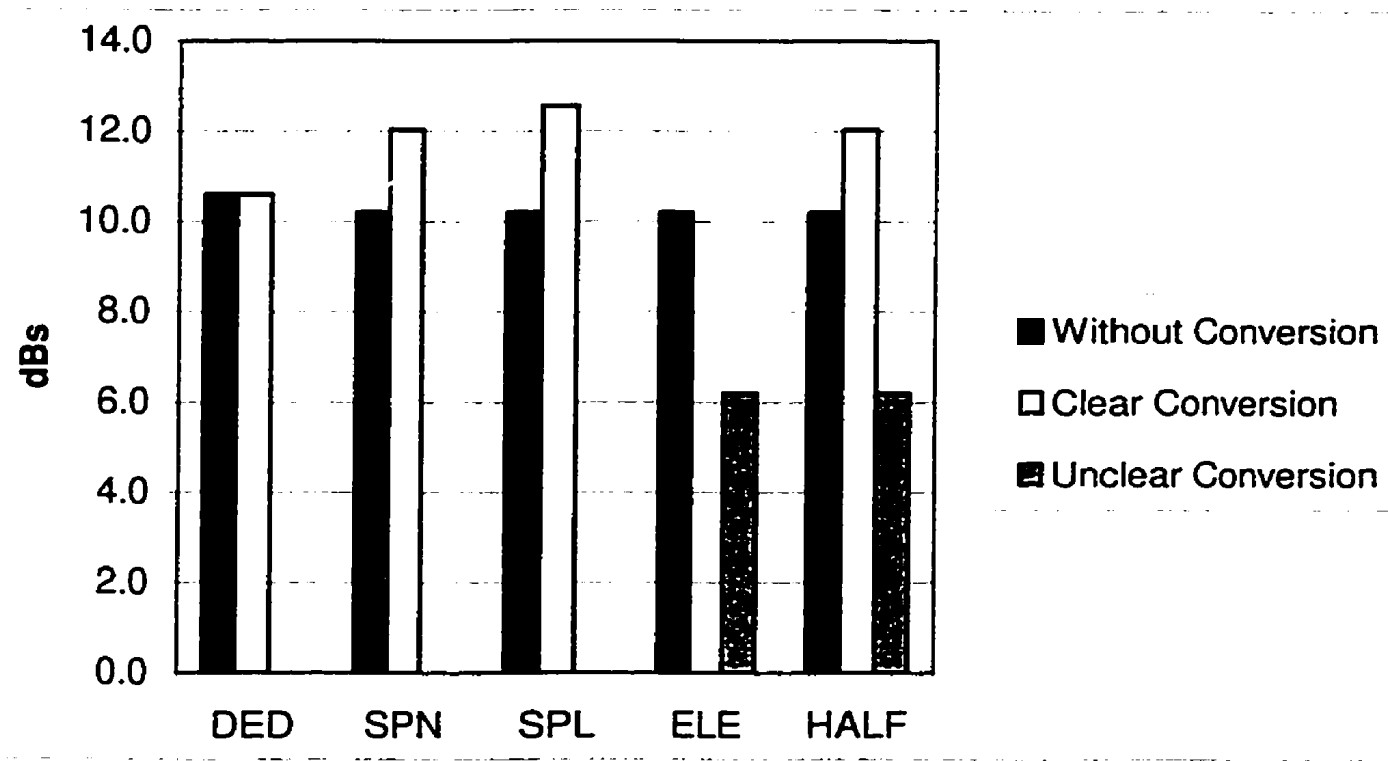

Figure 3-12: dB loss for a packet to pass through a switch

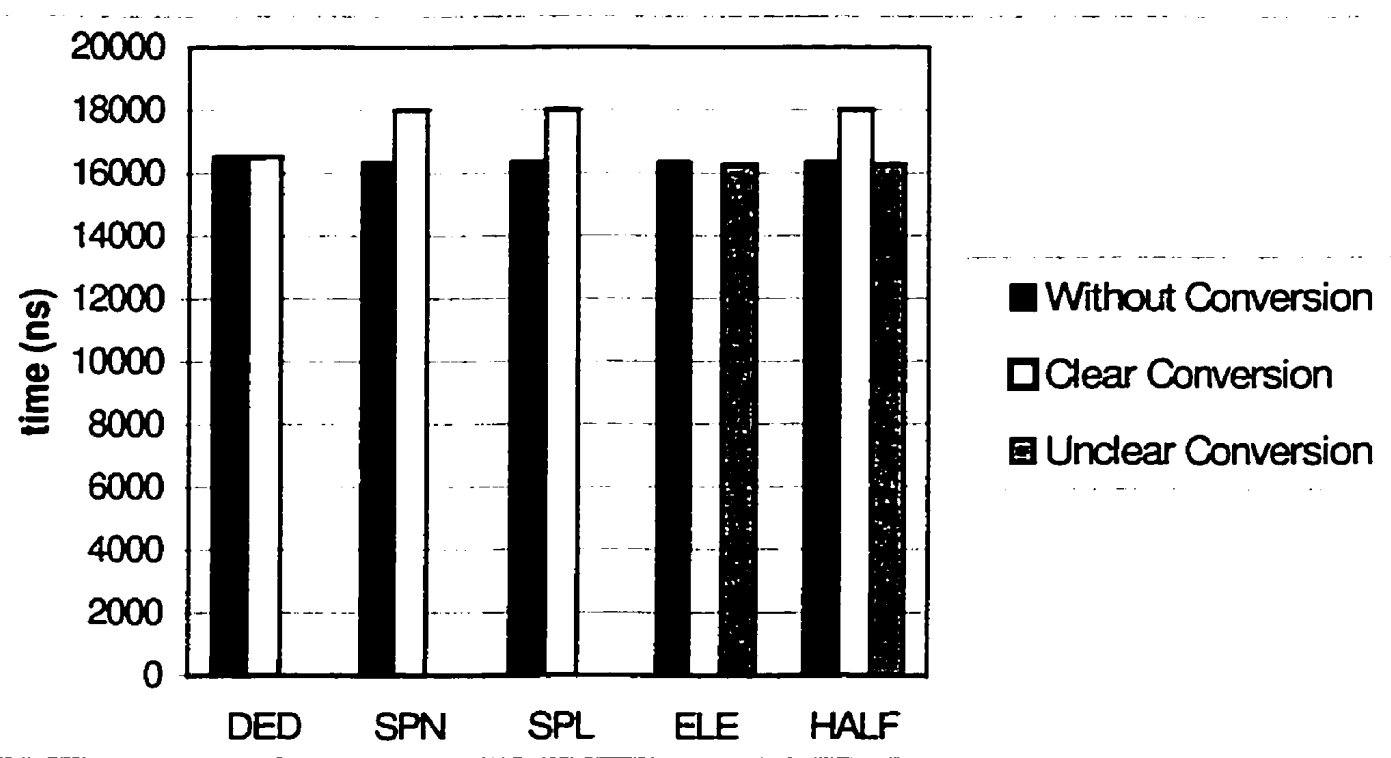

Figure 3-13: Processing time for a packet to pass through a switch 


\subsubsection{Discussion of Analysis}

As expected, when considering a packet that does not require conversion, each of the five switches performs equally. The only difference being that the dedicated wavelength convertible switch has a slightly higher attenuation and processing because the packets pass through a wavelength converter regardless whether conversion is required or not.

When looking at the route for clear conversion (i.e. no optical - electronic conversion), the dedicated wavelength convertible switch outperforms the share-per-link, share-per-node and half-clear wavelength convertible switches. This is due to the second optical switching stage in the latter two switches and the optical concentrators required in the share-per-link wavelength convertible switch. This second switching stage increases the attenuation and processing time associated with each packet. The loss incurred with the share-per-link wavelength convertible switch is slightly higher due to the optical concentrators required before the converter bank at each link. However, putting the dedicated wavelength convertible switch aside, these latter three switches perform on an equal footing with regards to clear conversion.

When looking at unclear conversion, the performance of the electronic and the half-clear wavelength convertible switches are similar, as expected. The question facing network designers would then become whether they prefer clear or unclear conversion. This is a performance issue and in most cases, clear conversion is preferred due to the qualities of clear wavelength conversion discussed earlier in this thesis. 
As each switch basically performs the same with a few minor differences, the evaluating criteria then becomes cost. When considering cost, the dedicated wavelength convertible switch will likely not be considered for widespread deployment simply because it is too expensive. With dedicated wavelength converters at each output link, costs rise dramatically compared with the other switch architectures.

Obviously, the electronic wavelength convertible switch is the cheapest. As there are no wavelength converters associated with this architecture, the costs can be kept to a minimum. This switch architecture can be argued as a cost-effective alternative to architectures employing wavelength converters [4]. However, many network engineers prefer clear conversion. The decision of which switch architecture to employ is generaliy a compromise.

This leaves us with the three architectures sharing conversion resources. Since the share-per-node and share-per-link architectures have the capability to convert every wavelength, it was expected that the costs associated with these switches would be higher than those of the half-clear wavelength convertible switch. With the limitation of only having the capability to convert half of the wavelengths in the system at each node, the half-clear wavelength convertible switch architecture has costs more closely related to that of the electronic wavelength convertible switch. Thus, this compromise of performance with cost can be beneficial to network engineers. The performance of the half-clear wavelength convertible switch is shown in the following two chapters to be equivalent to that of the dedicated wavelength convertible switch, proving that this switch is a very good, cost-effective alternative where clear conversion is preferred. 


\subsection{Chapter Summary}

This chapter introduces the half-clear wavelength convertible switch as an alternative to other wavelength convertible switch architectures. It investigated the five wavelength convertible switches in more detail. The node architecture was examined in a general sense, and then each wavelength convertible switch was investigated individually in Section 3.3.

The examination showed that the dedicated wavelength convertible switch had the best performance, but was very expensive. The half-clear wavelength convertible switch was shown to be a cost effective alternative. Further examination of the half-clear wavelength convertible switch will be conducted in Chapters 4 and 5 . 


\section{CHAPTER 4 - THE WAVELENGTH CONVERTIBLE OPTICAL NETWORK SIMULATOR (WCONSIM)}

\subsection{Introduction}

An algorithm was developed by Naas [14] entitled the Routing and Wavelength Assignment / Network Cost Optimization (RWANCO) algorithm. It details the operation of a wavelength routing network using the static-static model described in Section 2.4. This algorithm also calculates the optimum network cost associated with the network under study.

The RWA/NCO algorithm was used as a base in developing a new software package entitled the Wavelength Convertible Optical Network Simulator (WCONSIM). The cost optimization portion was removed, as a deterministic approach to network costs was done in this thesis. The RWA NCO algorithm had a small portion concerning wavelength conversion. As this is the main emphasis of this research, RWA routines were completely rewritten to reflect the emphasis on wavelength conversion in this research. 


\subsection{Assumptions}

To help facilitate the development of WCONSIM, some simplifying assumptions were made. Some of these assumptions were carried over from [14], others have been added specifically for WCONSIM. The assumptions are as follows:

- Physical Topology, $G_{p}$ : it is assumed that a single mode fibre backbone within the $N$ node network is already in existence. The physical topology is represented by one $N x N$ symmetric matrix, $\underline{D}$. $\underline{D}$ represents the distance of the physical connections, where each entry $D_{m n}$ is equal to the physical distance in kilometers between node $m$ and node $n$. If no physical connection exists between two nodes, $m$ and $n$, then $D_{m n}=$ $\infty$. The physical connectivity of the network can be obtained from the entries in $\underline{D}$. If $D_{m n}<\infty$, then there is a physical connection between node $m$ and $n$.

- Virtual Topology, $\underline{G}_{v}:$ it is assumed that virtual links are simplex. That is, a virtual link from node $a$ to node $b, v_{a b}$, does not share the same link as a virtual link from node $b$ to node $a, l_{b a}$.

- Modeling network traffic: it is assumed that a traffic matrix, $\underline{\Lambda}$, represents the longterm average of traffic requirements between each node in the network. The $(s, d)^{\text {th }}$ element, $\Lambda_{s d}$, represents the long term traffic average from node $s$ to node $d$.

- Time delay constraint: it is assumed that there is a limit to the amount of delay packets can experience during transmission represented by a global variable $t^{\max }$. 
- Virtual Link Capacity: it is assumed that all virtual links have the same capacity, $c$. This assumes that all virtual links have the same amount of bandwidth available. To reduce network sensitivity to traffic variations, the bandwidth available can be constrained by the factor $\rho_{\max }$, resulting in a capacity equal to $c \cdot \rho_{\max }$. In the simulations presented in the following chapters, $\rho_{\max }=1$.

- Non-bifurcated traffic: it is assumed that non-bifurcated traffic is used, and only one virtual link is established between any $s-d$ pair. With this in mind, it is clear that all traffic matrix elements have the following constraint: $\Lambda_{s d} \leq c \cdot \rho_{\max }$.

- Modeling queuing delay: virtual links are terminated at the interface part of the node architectures. As such, it is assumed that an output-buffered electronic switch, with infinite output buffer at all switch ports is used. Therefore, the network is modeled as a network of $M / D / 1$ queues with infinite buffers. The average queuing delay at a virtual link between node $a$ and node $b$, based on Kleinrock's independence assumption [28], is expressed as:

$$
E\left(t_{a h}^{Q}\right)=\frac{1}{\mu_{a b}-\hat{\lambda}_{a b}}\left(1-\frac{\hat{\lambda}_{a b}}{2 \mu_{a b}}\right)
$$

where $\hat{\lambda}_{u b}$ is the average arrival rate (Poisson) [cells/sec], or, for Poisson arrivals, it can also be viewed as the aggregate traffic on the virtual link $l_{a b}$, where $\hat{\lambda}_{a b}<c \cdot \rho_{\max }$, and $\mu_{a b}$ is the average service rate of the electronic switch part at node $a$ where the 
virtual link $l_{a b}$ originates. This can be calculated in cells/sec. It is also assumed that the port can fully utilize the virtual link capacity.

- Network cost model: it is assumed that the network cost is an aggregate cost of the WDM components in each of the network nodes. Not included in the cost calculations are:

- the long haul fibre links interconnecting the nodes since they already exist,

- the optical fibre cross-connects used inside the network nodes as they are very small and the additional cost added by these components in negligible,

- optical amplifiers since the optimum placement amplifiers is beyond the scope of this research,

- optical switching control. It is assumed that the optical switches are electronically controlled, and as such, the cost of the control equipment is negligible in the overall cost model, and

- electronic switches since they already exist.

- Network Reliability: it is assumed that reliability considerations regarding network or link failure are not considered.

- Node Complexity: it is assumed that there is no minimum WDM-hardware requirement at any node. However, upon addition of hardware elements, it is assumed that the full capacity of that element is installed. For example, if a multiplexer is used for only three virtual links, even though there are 16 wavelengths in the network, it is assumed that the multiplexer will be a 16-channel multiplexer as 
opposed to a 3-channel multiplexer. This allows for future upgrades and reconfigurations if necessary and is in line with current engineering practice.

- Wavelength limits: it is assumed that there is a technological limit on the number of wavelengths that each fibre can carry. This limit is represented by the global variable $W_{\max }$

\subsection{High Level Description of Simulator}

A high-level block diagram of the Wavelength Convertible Optical Network Simulator is shown in Figure 4-1. It is composed of three different functional modules: the initialization module, the RWA module, and the statistics gathering module.

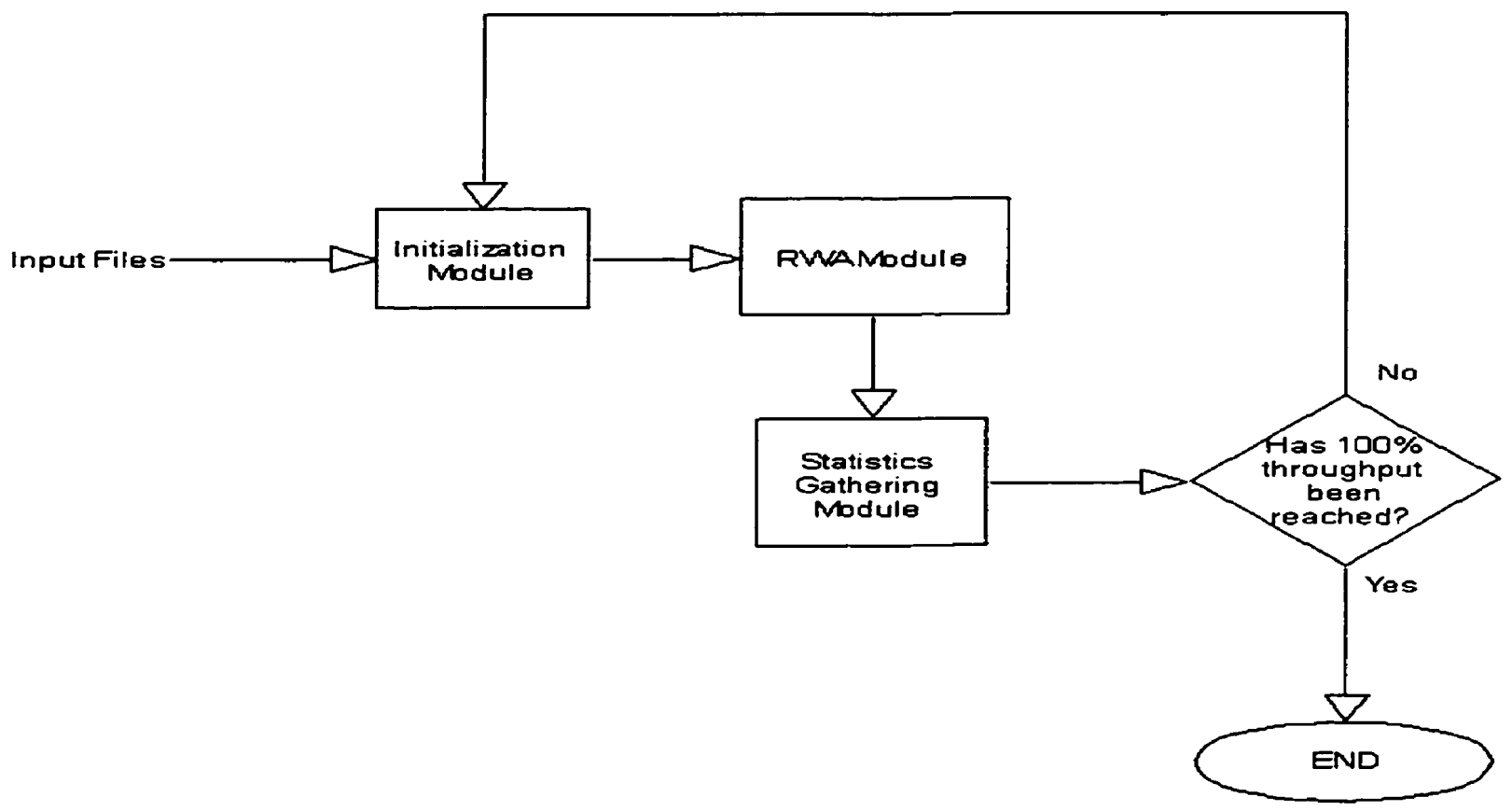

Figure 4-1: WCONSIM Block Diagram 
Input parameters are supplied to the program through two input files: the network physical connectivity $\left(G_{p}\right)$, and the link distances $(\underline{D})$. Other input parameters are physically set within the code prior to execution or specified within the program during execution. At the end of execution, different statistical output files are generated containing various performance measures. The following sections describe in some detail each of the three main modules of the WCONSIM application.

\subsection{Initialization Module}

The initialization module is composed of two main sections: the variable initialization and the traffic matrix generation. Each of these sub-modules is described in the following sections.

\subsubsection{Variable Initialization}

In this sub-module, global variables that impact the operation of the simulator are set. These include physical connectivity and distance matrices, which are read in from files. Hardware resources available at each network node are also determined. Hardware resources are dependent on the number of wavelengths in use, and as such, this module is run again each time the number of wavelengths is increased. Hardware resources include:

- Demultiplexers/Multiplexers: The maximum complexity model is given by

$$
\text { Complexity }_{D E M U X \mid M U X}^{\max }=\left\{\begin{array}{l}
\text { Number }: \Delta_{p}^{i} \\
\operatorname{Size}(D E M U X):|x| W \mid \quad \forall i \in\{0,1, \ldots N-1\} \\
\operatorname{Size}(M U X):|W| x \mid
\end{array}\right.
$$


- Optical-Space Division Switches: The maximum complexity is given by

$$
\text { Complexity } \text { OpricalSwich }_{\max }^{\max }=\left\{\begin{array}{l}
\text { Number }:|W| \\
\text { Size }: 8 x 8 \text { if } \Delta_{p}^{i} \leq 4 \\
\text { Size }: 16 x 16 \text { if } \Delta_{p}^{i} \geq 5
\end{array}\right.
$$

- Transmitters/Receivers: The maximum complexity is given by

$$
\text { Complexity } \max _{T \times R x}^{\max }=\left\{\begin{array}{l}
\text { Number }:|W| \cdot \Delta_{p}^{i} \text { if multiple-fibre architecture used } \\
\text { Number }:|W| \text { otherwise }
\end{array}\right.
$$

- Wavelength Converters: The maximum complexity is given by

$$
\text { Complexity } w_{W C}^{\max }=\left\{\begin{array}{l}
\text { Number }:|W|-\Delta_{p}^{i} \quad \text { if dedicated switch used } \\
\text { Number }: \frac{|W|}{2} \text { if half-clear switch used }
\end{array}\right.
$$

\subsubsection{Traffic Matrix Generation}

A new traffic matrix is generated at the beginning of each simulation run. For each run, a new seed is used for the random number generator to ensure a different traffic matrix is randomly generated. The application runs the simulation 50 times and collects the averages in the statistics generating module. 
Two types of traffic matrices are possible: Uniform and Non-Uniform:

- Uniform: in this type of traffic matrix, the traffic elements are uniformly and randomly distributed over the range $(0, x)$, where $x$ is the maximum offered traffic load for each $s-d$ pair and $x \leq c$.

- Non-Uniform: in this type of traffic matrix, $(100-F) \%$ of the traffic elements are uniformly and randomly distributed over the range $(0, x)$, where $x$ is the maximum offered traffic load for each $s-d$ pair and $x \leq c . F \%$ of the elements are uniformly and randomly distributed over the range $(0, y)$, where $y \leq x$. Increasing the value of $F$ would result in the traffic becoming more concentrated among a few s-d pairs, decreasing the value of $F$ would result in the traffic becoming more evenly distributed. If $F=0 \%$, then a uniform traffic matrix is generated.

\subsection{RWA Module}

In the RWA Module, two problems are solved for a given $|W|$ : first, finding a virtual connection between a source node and destination node and assigning a wavelength to that connection, and second, routing traffic over the resulting virtual connections. In WCONSIM, these two sub-problems are solved simultaneously. Since $s-d$ pairs are sorted prior to assigning virtual links (see policies below), traffic can be routed at the same time as virtual links and wavelengths are assigned. 
There are three methods of establishing a virtual connection between any two nodes in a network. These methods are:

- Continuous Clear Lightpath: This type of connection refers to a connection that utilizes the same wavelength throughout its path (continuous) and remains in the optical domain (clear). That is, the optical switches in the intermediate nodes are set in bypass mode for the signal (see Section 3.3.1). This connection satisfies the wavelength continuity constraint. A packet travelling on a continuous clear lightpath will not experience delays associated with electro-optic conversion or queuing. A downside to this type of connection would be the high hardware cost. Each connection requires a dedicated transmitter, receiver, optical switch ports and multiplexer/demultiplexer channels.
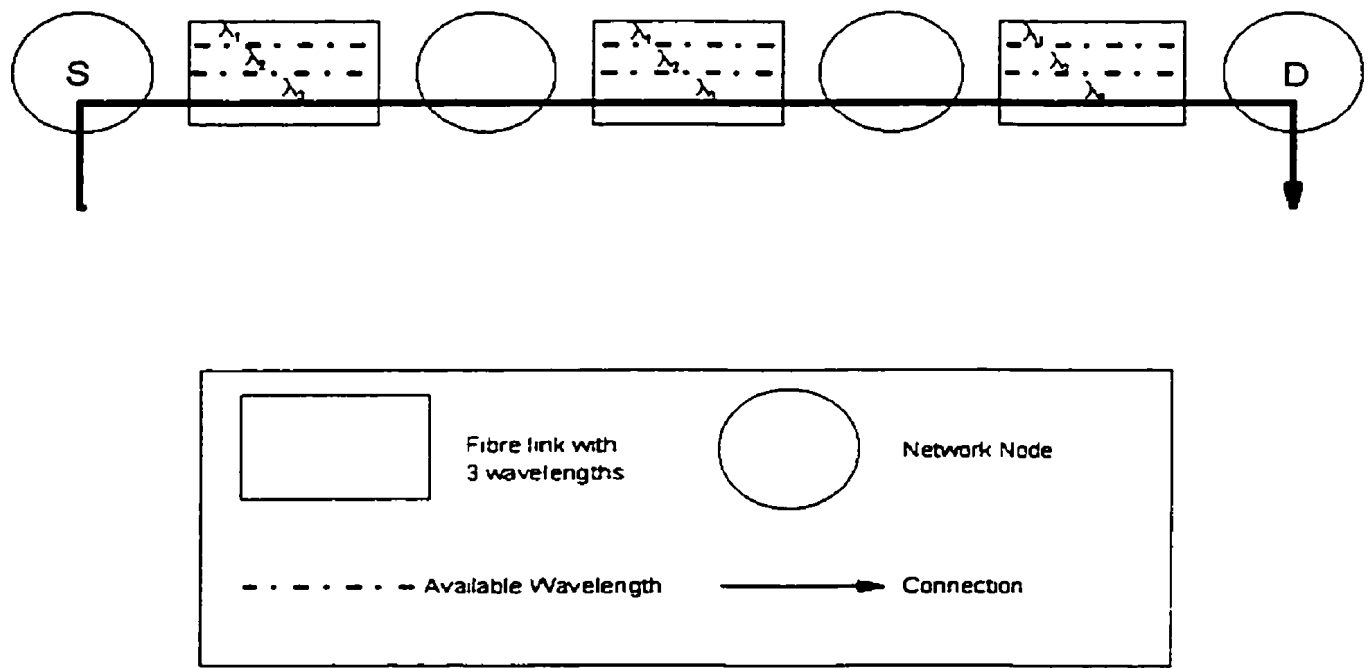

Figure 4-2: Illustration of a Continuous Clear Lightpath 
- Non-Continuous Clear Lightpath: This type of connection refers to a connection that may utilize a wavelength converter along the route to change the wavelength of the signal (non-continuous), but remains in the optical domain (clear). The wavelength continuity constraint is not satisfied with this type of connection. A packet travelling on a continuous clear lightpath will also not experience delays associated with electro-optic conversion or queuing. However, since a wavelength converter is required, costs can be expected to be more than the continuous clear lightpaih connection.
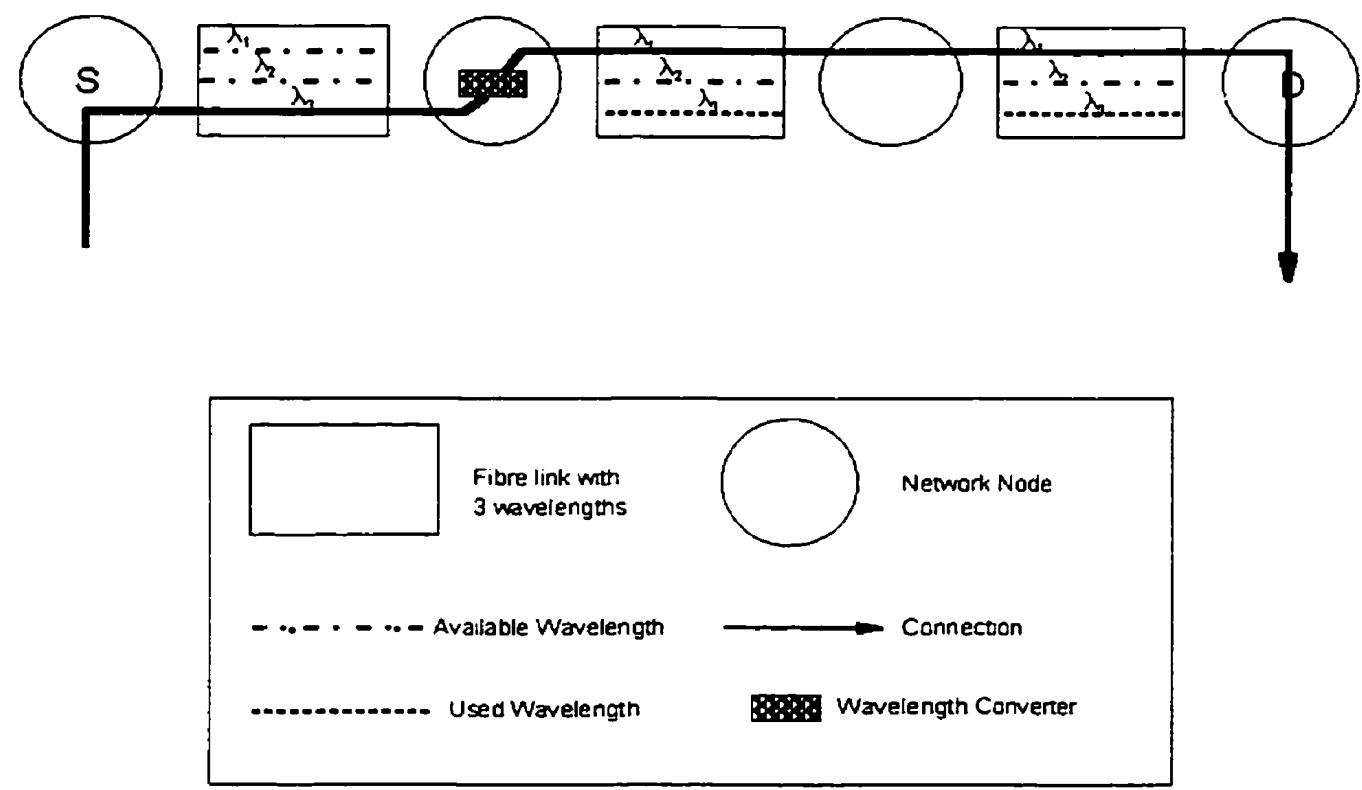

Figure 4-3: Illustration of a Non-Continuous Clear Lightpath 
- Unclear Lightpath: This type of connection takes advantage of existing clear lightpaths that have previously been set up within the network. In this type of connection, the traffic is combined with other traffic already travelling on the existing lightpaths. However, to switch lightpaths at an intermediate node, the electronic switching matrix is required to route the signal from one lightpath to another. The advantage of using this type of connection is the cost as no new optical hardware is required. This type of connection also takes advantage of the bandwidth available on existing connections. However, packets will experience higher delays due to the electro-optic conversion and queuing within electronic switching matrix at the intermediate nodes.
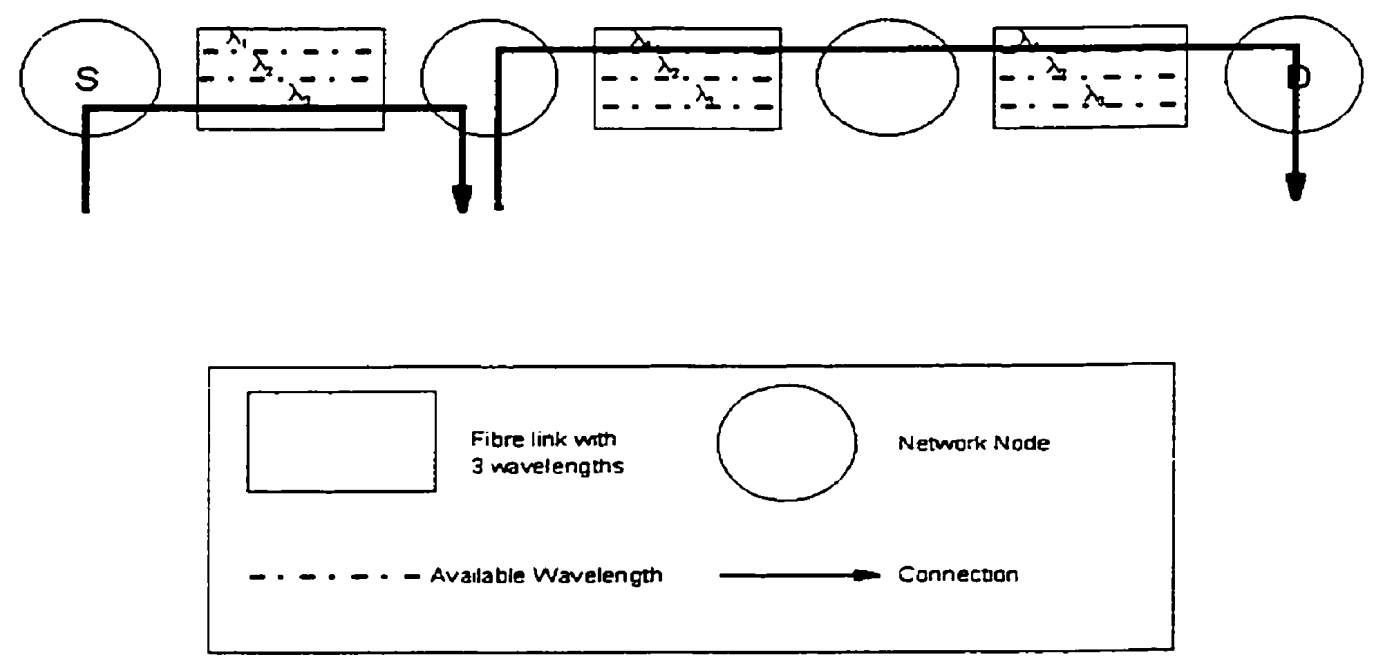

Figure 4-4: Illustration of an Unclear Lightpath 
It is clear that a different virtual topology will be obtained depending on the order the $s$ - $d$ pairs are served. As such, four policies are suggested for ordering s-d pairs:

- Policy 1: Where $s$-d pairs are sorted in an increasing order of their traffic demands. The idea behind this policy is that any new virtual links created will have high residual capacity, enabling reuse of existing links as much as possible for other $s-d$ pairs.

- Policy 2: Where $s$ - $d$ pairs are sorted in a decreasing order of their traffic demands. The idea behind this policy is that routing the majority of traffic in one virtual link may lower the number of virtual links required.

- Policy 3: Where $s$ - $d$ pairs are sorted in a decreasing order of their least number of physical hops required. If more than one pair has the same number, they are sorted randomly. The idea behind this policy is that the traffic travelling the furthest physical distance is routed first, potentially decreasing the number of wavelength conversions required enroute.

- Policy 4: Where $s$ - $d$ pairs are sorted in an increasing order of their least number of physical hops required. If more than one pair has the same number, they are sorted randomly. The idea behind this policy is to minimize the physical hop distance of the connections.

During execution of the simulator, $s-d$ pairs are sorted according to one of the policies described above, then connections are attempted to be made in the following order: 
Step 1. Unclear Lightpath. Attempts are made to route the traffic for this $s-d$ pair utilizing any existing lightpaths first. All wavelengths are considered when examining existing lightpaths for use. The path found must adhere to the delay restrictions (delay must be under the set maximum allowable delay). If no path is found, then go to step 2 .

Step 2. Continuous Clear Lightpath. Creation of a new continuous clear lightpath is attempted to connect this $s-d$ pair. All wavelengths are examined to see if a path can be made, with priority given to the lower wavelengths (i.e. if a path exists on both $\lambda_{1}$ and $\lambda_{2}$, then the connection will be made using $\lambda_{\mathrm{I}}$ ). The path found must adhere to the delay restrictions (delay must be under the set maximum allowable delay). If creation of a clear continuous lightpath is not possible, go to step 3 .

Step 3. Non-Continuous Lightpath. Creation of a new non-continuous lightpath is attempted to connect this $s-d$ pair. All wavelengths are examined to see if a path can be made, with priority given to the path with the least number of wavelength conversions required. The path found must adhere to the delay restrictions (delay must be under the set maximum allowable delay). If creation of a non-continuous clear lightpath is not possible, there is no way to route the traffic for this $s-d$ pair.

Once the entire sorted list has been traversed, the statistics gathering module is called. The amount of traffic successfully routed during that pass is calculated. If the throughput is less than $100 \%$, the number of wavelengths available is increased by one and the sorted list is traversed again. This process repeats until $100 \%$ throughput is attained. 


\subsection{Statistics Gathering Module}

The statistics gathering module is invoked after each traversal of the sorted $s-d$ pair list. This relates to one call to the statistics gathering module each time the number of wavelengths has increased. The main purpose of this module is to gather statistics regarding the performance of the network for each $|W|$. This section will define the mathematical functions used in generating the network statistics calculated.

- Network Throughput. This metric measures the total amount of traffic that has been successfully routed. It is given by:

Network Throughput $=\frac{\sum_{s, d}^{s \neq d} \Lambda_{s d} \cdot \mathrm{Y}_{s d}}{\sum_{s, d}^{s \neq d} \Lambda_{s d}}$

where $\underline{\Lambda}=$ traffic matrix, and

$\underline{Y}=$ entries indicate traffic successfuiiy routed

- Average Network Delay. This metric is the average delay experienced by a packet in the network. It is a combination of queuing and propagation delay and is given by:

$$
\begin{aligned}
& E(t)=E\left(t^{p}\right)+E\left(t^{Q}\right) \\
& E(t)=\frac{1}{\gamma} \sum_{k=0}^{\text {wrti }} \sum_{i, j}^{i \neq j} V_{i j}^{k} \hat{\lambda}_{i j} \cdot\left(t_{i j}^{p}+\frac{1-\hat{\lambda}_{i j} / 2 c}{c-\hat{\lambda}_{i j}}\right) \\
& \text { where } \gamma=\sum_{s, d}^{s \neq d} \Lambda_{s d} \cdot Y_{s d}, \\
& V^{k}=\text { virtual connectivity on } \lambda_{k}, \text { and } \\
& \hat{\lambda}_{i j}=\text { aggregate traffic on link } l_{i j}
\end{aligned}
$$


- Average Virtual Link Utilization. This metric measures the virtual link utilization averaged over all the virtual links in the network. It is given by [14]:

$$
\begin{aligned}
& E(\rho)=\frac{1}{|V|} \sum_{k=0}^{W / 1} \sum_{i, j}^{1 \neq j} \rho_{i j} \cdot V_{i j}^{k} \\
& \text { where }|V|=\sum_{i, j}^{i \neq j} \sum_{k=0}^{W W-1} V_{i j}^{k}, \text { and } \\
& \rho_{i j}=\frac{\hat{\lambda}_{i j}}{c}
\end{aligned}
$$

- Average Electronic Hops. This metric calculates the average number of electronic hops required by each connection and is given by [14]:

$$
E(h)=\frac{1}{\gamma} \sum_{k=0}^{|W|-\mid i \neq i} \sum_{i, j}^{\dot{\lambda}_{i j}} \cdot V_{i j}^{k}
$$

- Average Wavelength Utilization. This metric calculates the fraction of used wavelength channels per fibre. It is given by [14]:

$$
\begin{aligned}
& E(w)=\frac{1}{|P| \cdot|W|} \sum_{i, j}^{i \neq j}\left|W_{i j}\right| \\
& \text { where }|P|=\sum_{i, j}^{i \neq j} P_{i j}, \text { and }
\end{aligned}
$$

$P_{i j}=$ connectivity between node $i$ and $j$ (derived from distance matrix) 
- Number of Wavelength Converters. This metric measures the total number of wavelength converters that are in use in the network. It is given by:

$$
\Psi=\sum_{i=0}^{N-1} \bar{\lambda}_{i}
$$

where $\bar{\lambda}_{i}=$ number of converters in use at node $i$

\subsection{Software Verification and Validation}

Verification is determining that a simulation program performs as intended. Numerous tests are run in a test or simulated environment to prove the correctness of the simulation model [29]. Validation is concerned with determining if the conceptual simulation model (as opposed to the computer program) is an accurate representation of the real-world system under study [29]. Numerous steps were taken to perform verification and validation of the wavelength convertible optical switch simulator as detailed below.

The modular design of the simulation software enables each module to be tested independently. The software has a number of monitoring functions to enable testing in a controlled environment. When active, the monitoring mode outputs additional files, which include the traffic matrix, the sorted $s-d$ pairs list and the propagation delay matrix. This enables independent verification of the modules.

The WCONSIM application simulated a 7-node test network as shown in Figure 4-5. The distance matrix is also shown in Figure 4-5 for the test network. 


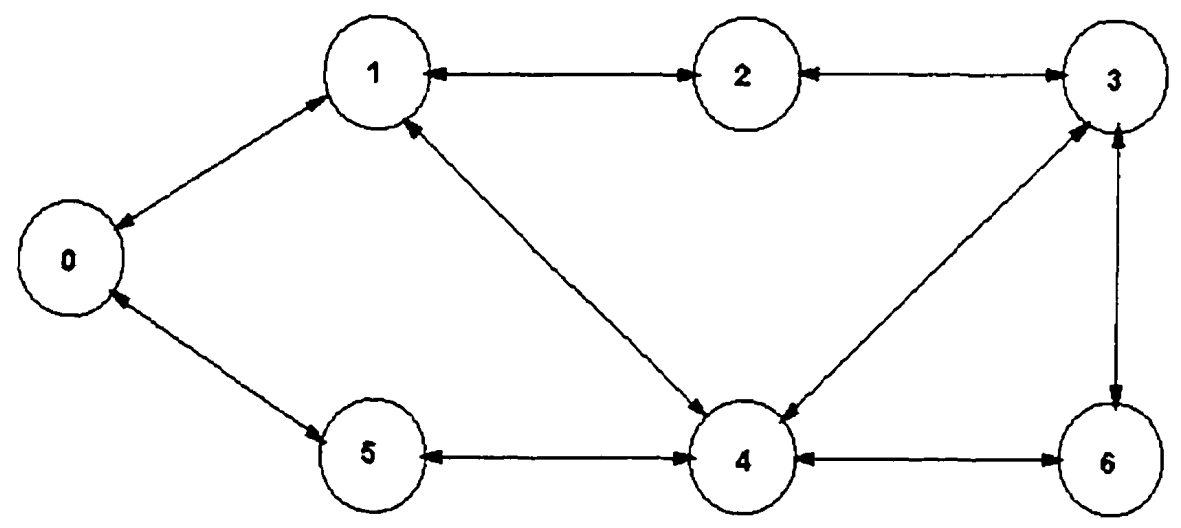

$$
\underline{D}=\left[\begin{array}{ccccccc}
- & 100.2 & \infty & \infty & \infty & 150.6 & \infty \\
100.2 & - & 200.7 & \infty & 400.3 & \infty & \infty \\
\infty & 200.7 & - & 170.1 & \infty & \infty & \infty \\
\infty & \infty & 170.1 & - & 90.9 & \infty & 100.1 \\
\infty & 400.3 & \infty & 90.9 & - & 160.7 & 110.8 \\
150.6 & \infty & \infty & \infty & 160.7 & - & \infty \\
\infty & \infty & \infty & 100.1 & 110.8 & \infty & -
\end{array}\right]
$$

Figure 4-5: The 7-node test network and its distance matrix

Using this test network, the simulator was run with the monitoring function active. Using the traffic matrix generated, and the sorted $s-d$ pair lists, independent verification and validation of the results using pencil and paper methods were conducted, and compared with the output of the simulator. This process was then repeated using a larger network, and finally the NSF Network used in reporting results in the next chapter. 
In each case, the results output by the simulator were equivalent to the results obtained through manual processes. This would suggest that the WCONSIM application was performing as expected and was an accurate representation of the networks under study. 


\section{CHAPTER 5 - NUMERICAL RESUL TS AND ANALYSIS}

This chapter presents and analyses the results obtained from the various simulation runs using the WCONSIM application. Figures are labeled according to the data they present.

In each case, graphs represent an average over 50 independent simulation runs. Confidence intervals of $95 \%$ are represented on each graph where appropriate. Figures depicting costs are generated using deterministic data as detailed in earlier chapters.

For results from Non-Uniform traffic matrices, a value of $F=75 \%$ was used in generating the traffic matrix. Three main metrics are represented in the figures presented in this chapter:

- cost

- throughput

- average packet delay

Two different network topologies were used in generating results for comparison:

The National Science Foundation (NSF) Network, and the European Optical Network (EON). These two networks, and their corresponding results, will be presented separately. 


\subsection{The NSF Network}

In this section, the NSF Network topology was used as the base for comparison. The following input-data was used for this investigation:

- Physical Topology: The 1991 14-node NSF Network as shown in Figure 5-1 [30]. The distances (in $\mathrm{km}$ ) of the physical links between the nodes are given in the following matrix:

$$
\underline{D}=\left[\begin{array}{cccccccccccccc}
0 & 1173 & 1677 & \infty & \infty & \infty & \infty & 2682 & \infty & \infty & \infty & \infty & \infty & \infty \\
1173 & 0 & 671 & 841 & \infty & \infty & \infty & \infty & \infty & \infty & \infty & \infty & \infty & \infty \\
1677 & 671 & 0 & \infty & \infty & \infty & 2011 & \infty & \infty & \infty & \infty & \infty & \infty & \infty \\
\infty & 841 & \infty & 0 & 671 & \infty & \infty & \infty & 2178 & \infty & \infty & \infty & \infty & \infty \\
\infty & \infty & \infty & 671 & 0 & 670 & 1342 & \infty & \infty & \infty & \infty & \infty & \infty & \infty \\
\infty & \infty & \infty & \infty & 669 & 0 & \infty & 840 & \infty & \infty & \infty & \infty & \infty & \infty \\
\infty & \infty & 2011 & \infty & 1342 & \infty & 0 & \infty & \infty & 1174 & \infty & \infty & \infty & 2010 \\
2682 & \infty & \infty & \infty & \infty & 840 & \infty & 0 & \infty & \infty & 839 & \infty & \infty & \infty \\
\infty & \infty & \infty & 2178 & \infty & \infty & \infty & \infty & 0 & \infty & \infty & 672 & 838 & \infty \\
\infty & \infty & \infty & \infty & \infty & \infty & 1174 & \infty & \infty & 0 & 838 & \infty & \infty & \infty \\
\infty & \infty & \infty & \infty & \infty & \infty & \infty & 839 & \infty & 838 & 0 & 334 & 336 & \infty \\
\infty & \infty & \infty & \infty & \infty & \infty & \infty & \infty & 672 & \infty & 334 & 0 & \infty & 337 \\
\infty & \infty & \infty & \infty & \infty & \infty & \infty & \infty & 838 & \infty & 336 & \infty & 0 & 168 \\
\infty & \infty & \infty & \infty & \infty & \infty & 2010 & \infty & \infty & \infty & \infty & 337 & 168 & 0
\end{array}\right]
$$

- Traffic: Both uniform and non-uniform traffic was considered.

- Node architecture: No wavelength conversion, full conversion and half conversion (the half-clear wavelength convertible switch) were considered.

- A delay constraint of $50 \mathrm{~ms}$ was imposed.

- No constraint on the virtual link capacity utilization was imposed. 
Results for this network are presented in Figure 5-2 - Figure 5-19. In Figure 5-2 and Figure 5-3, the $x$-axis is labeled with the routing scheme used, where the first number refers to the routing policy $(1-4)$ and the second letter refers to full (F), no $(N)$ or half $(H)$ conversion.

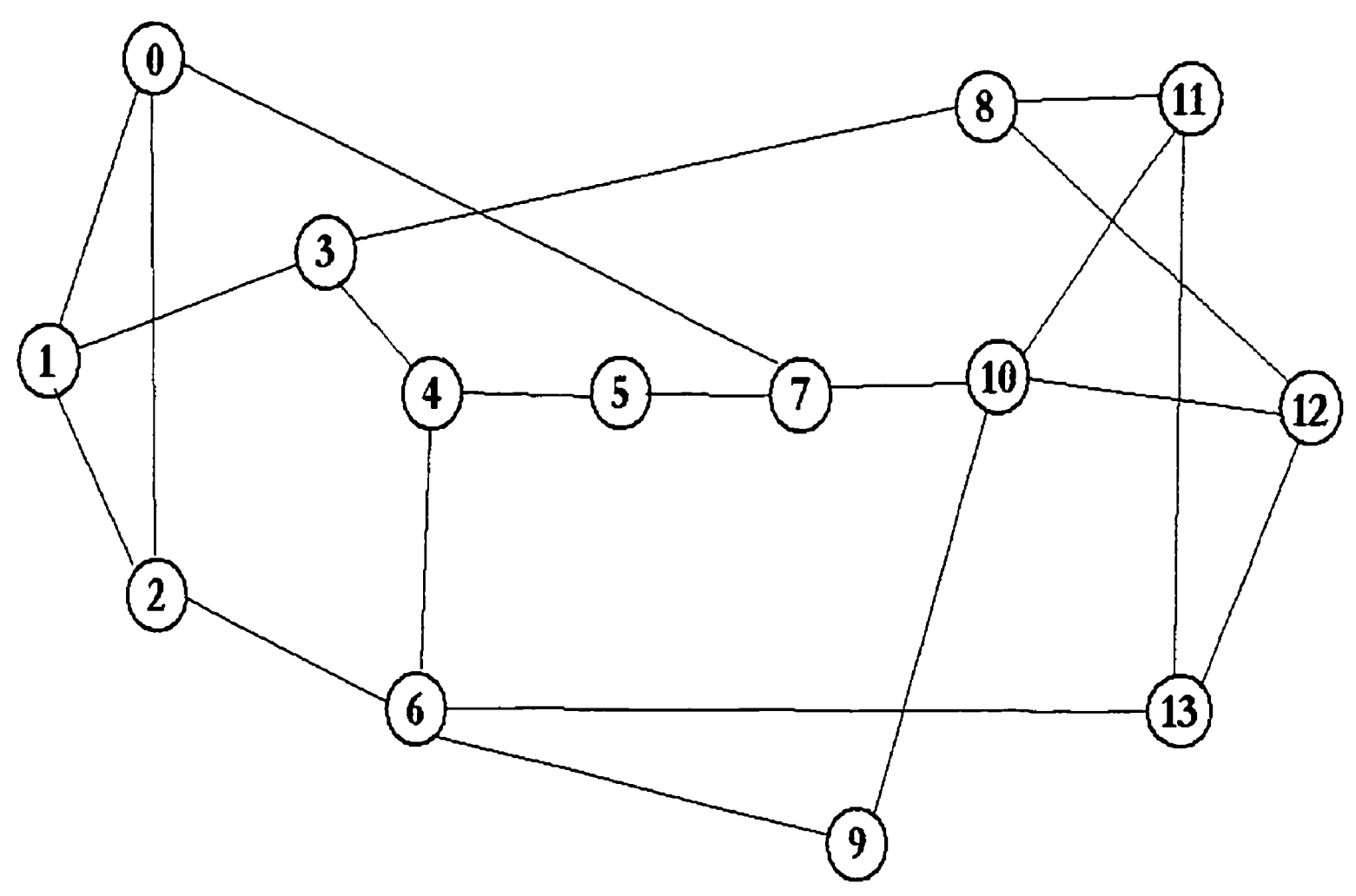

Figure 5-1: The 14 Node NSF Network 

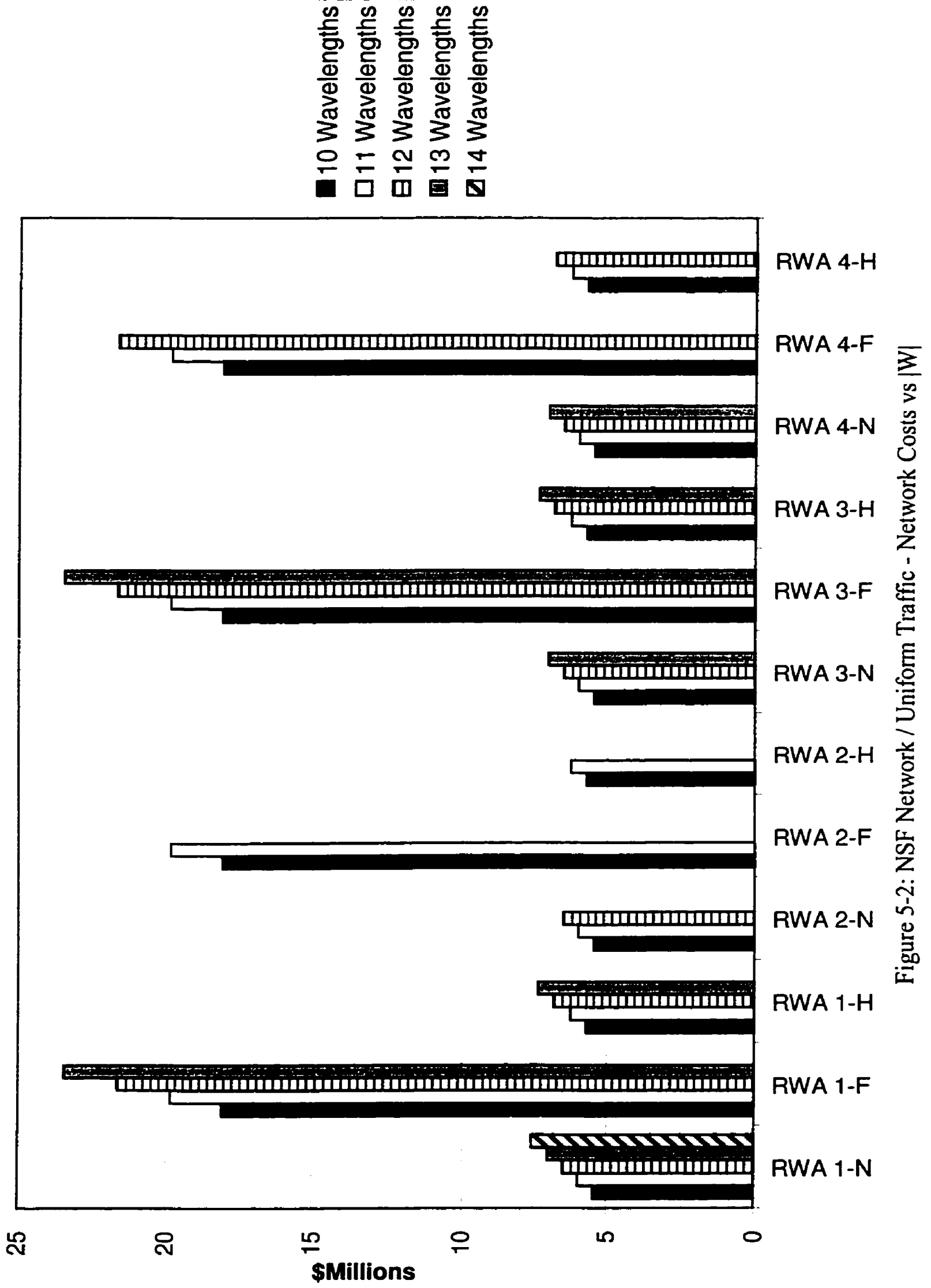

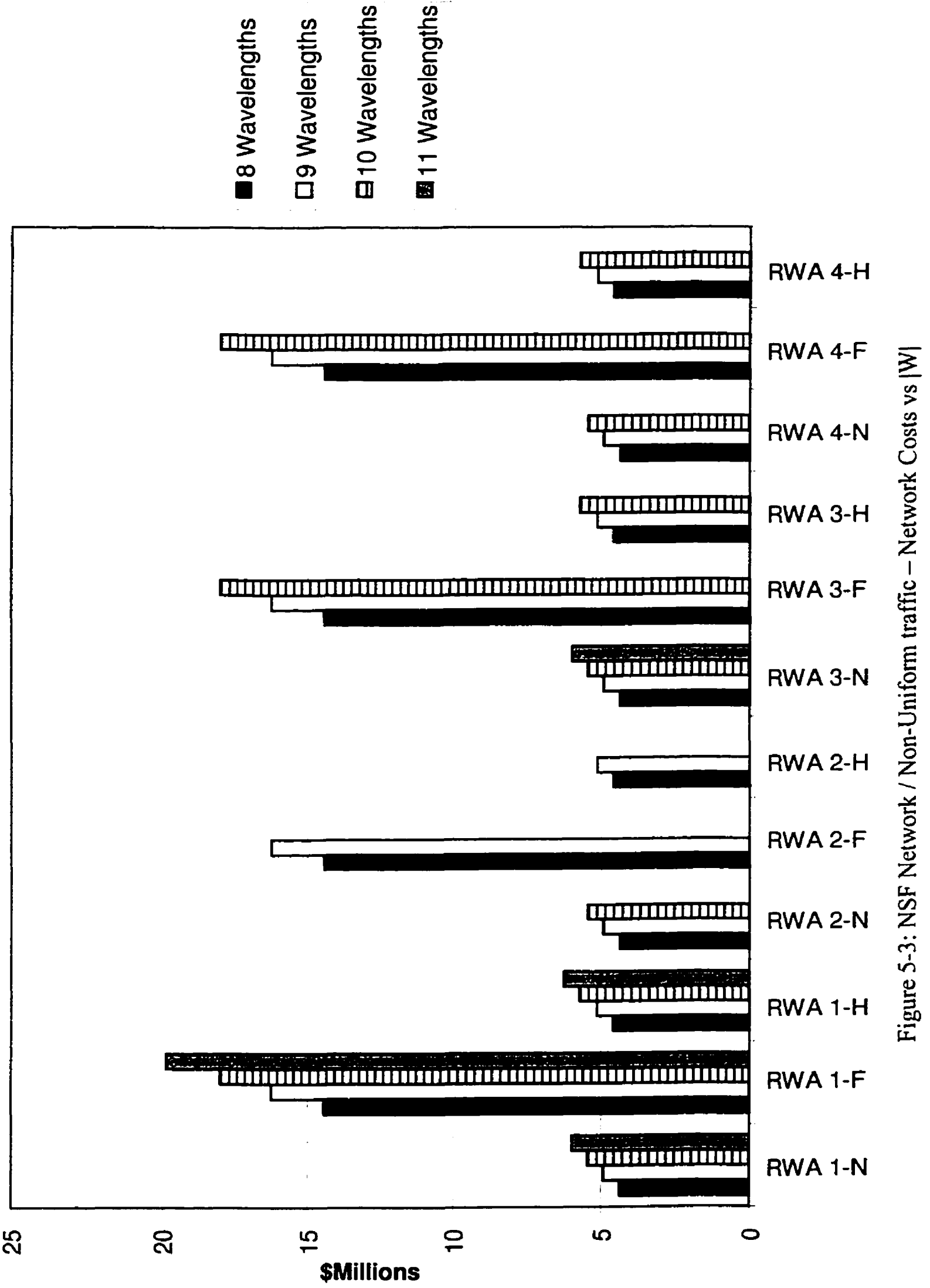


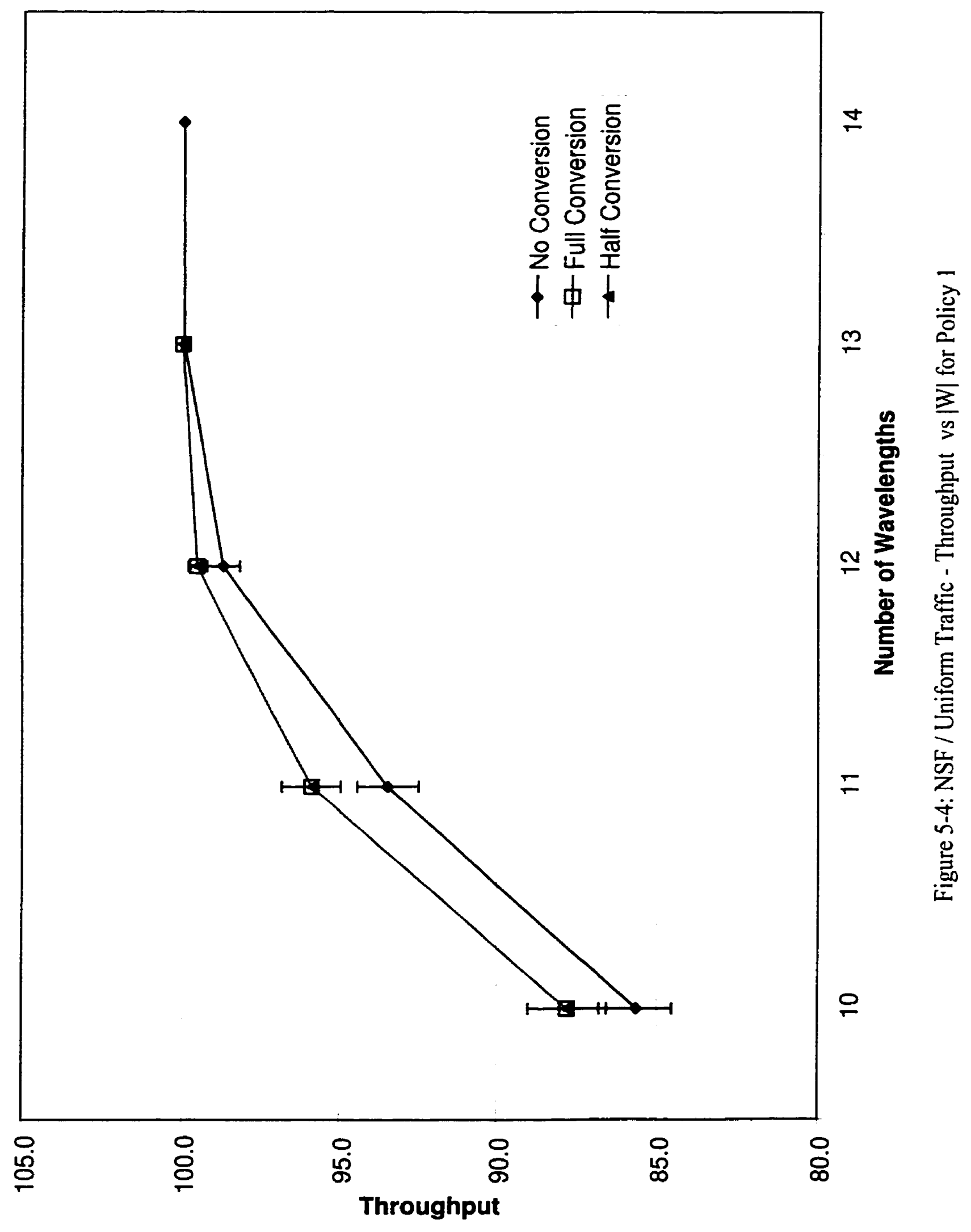




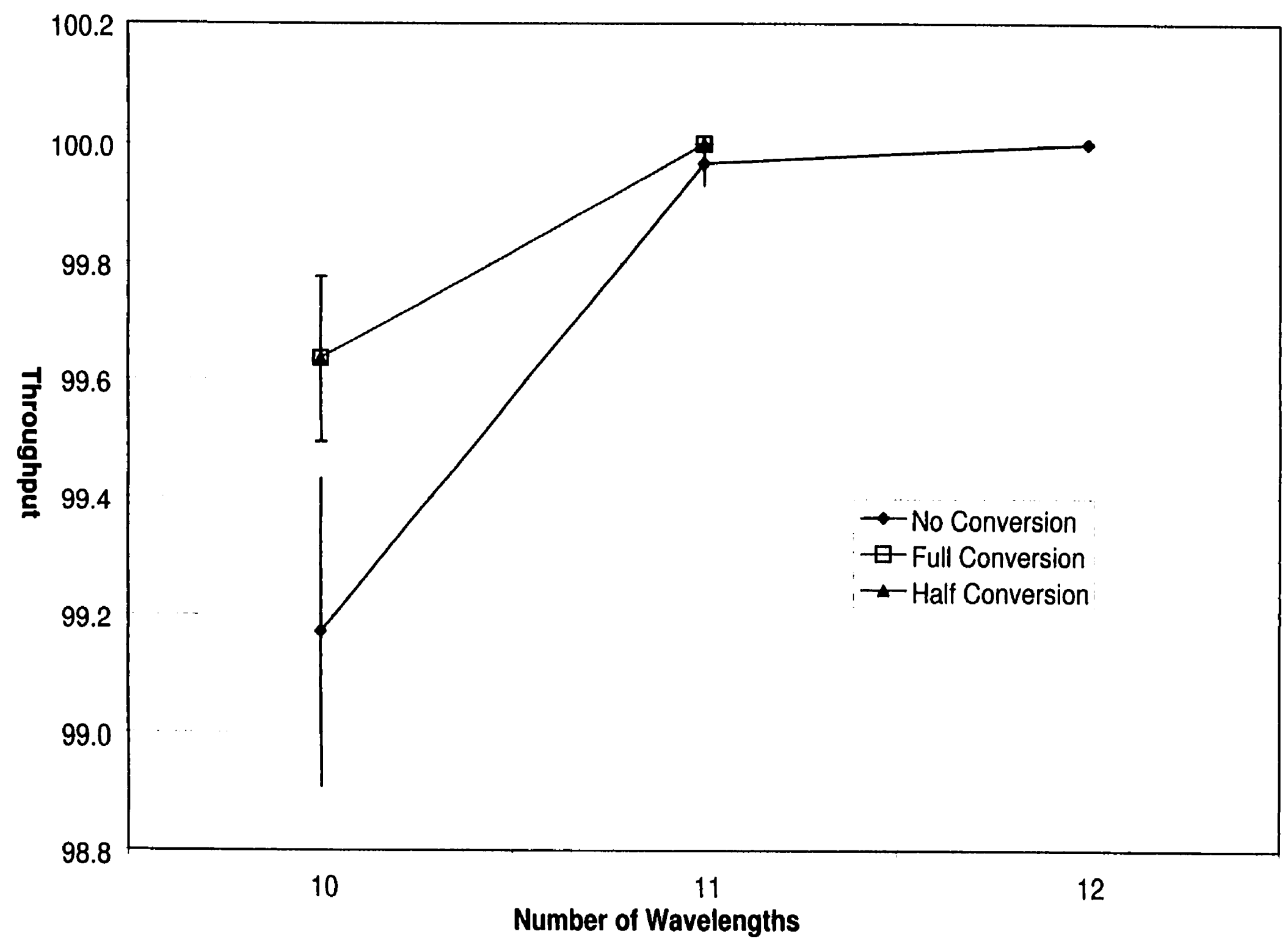

Figure 5-5: NSF / Uniform Traffic - Throughput vs |W| for Policy 2 


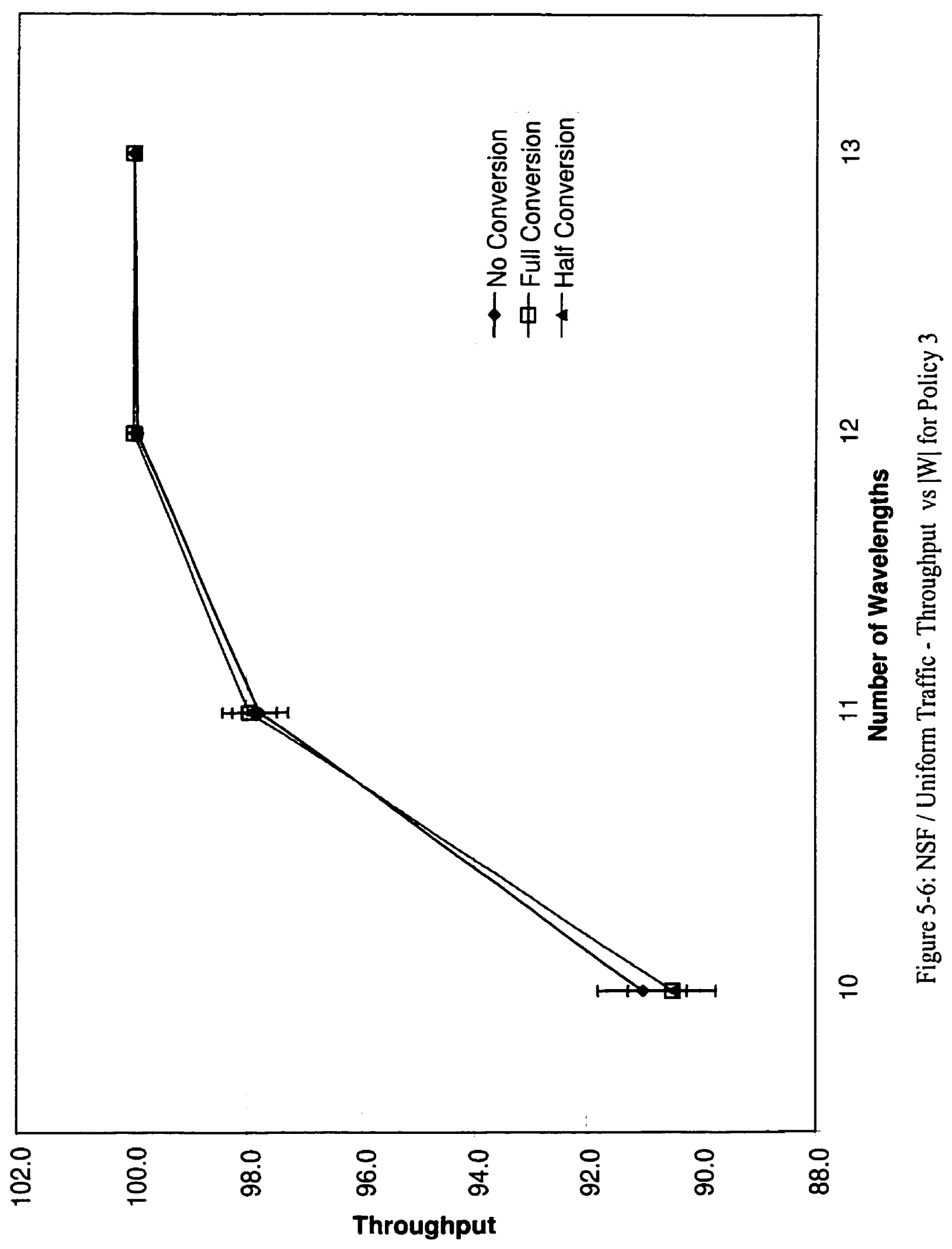




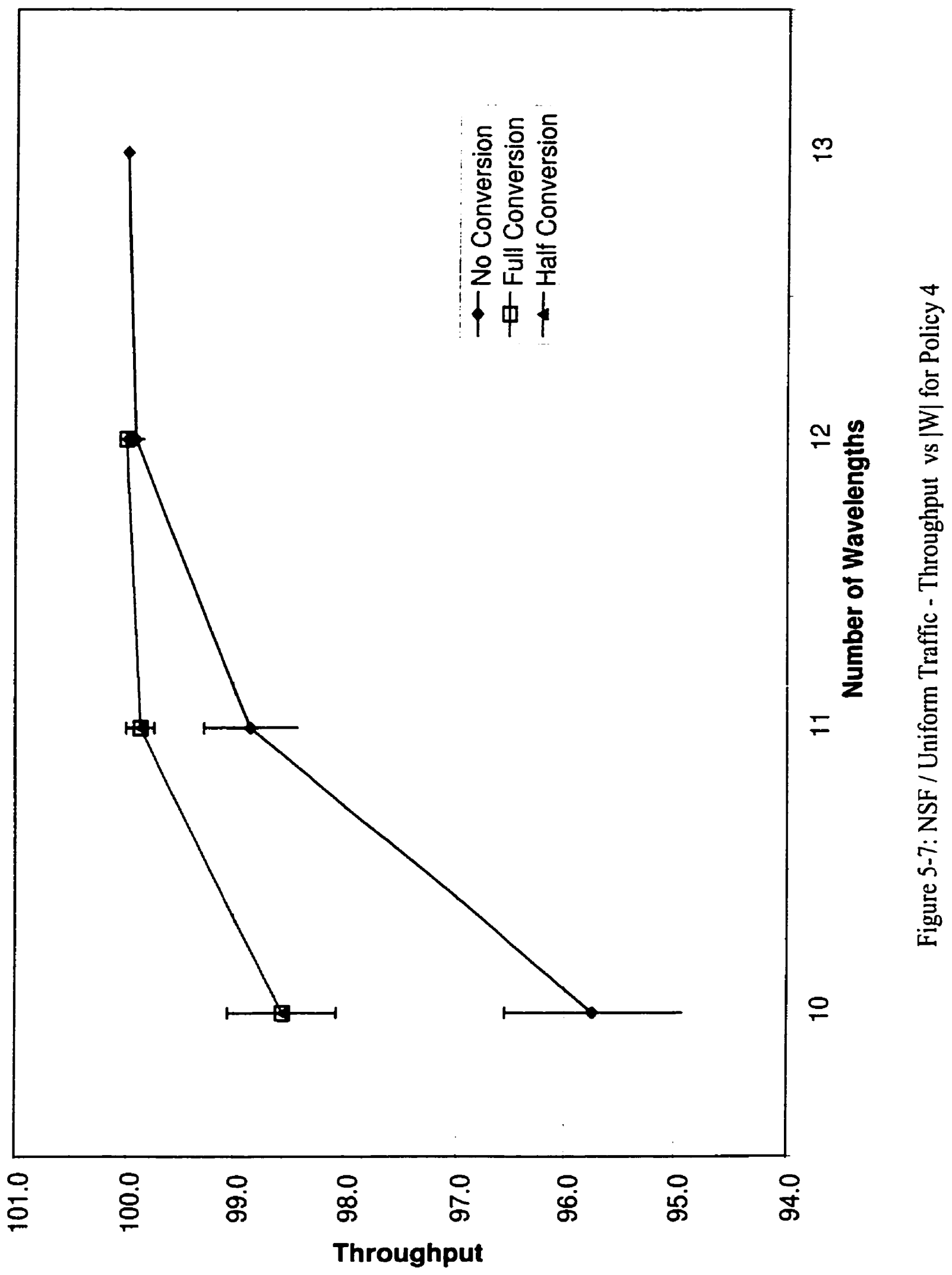




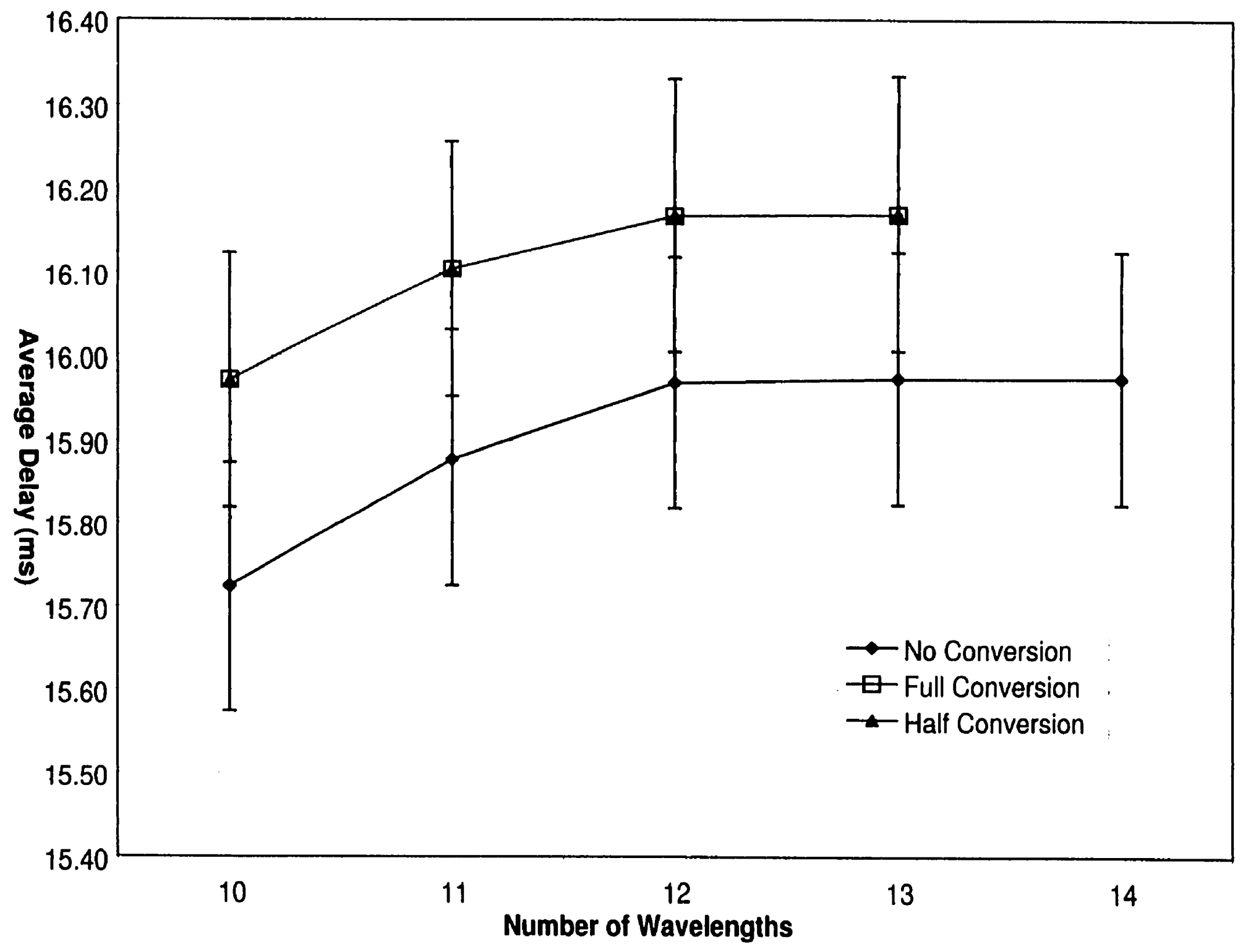

Figure 5-8: NSF / Uniform Traffic - Delay vs |W| for Policy 1 


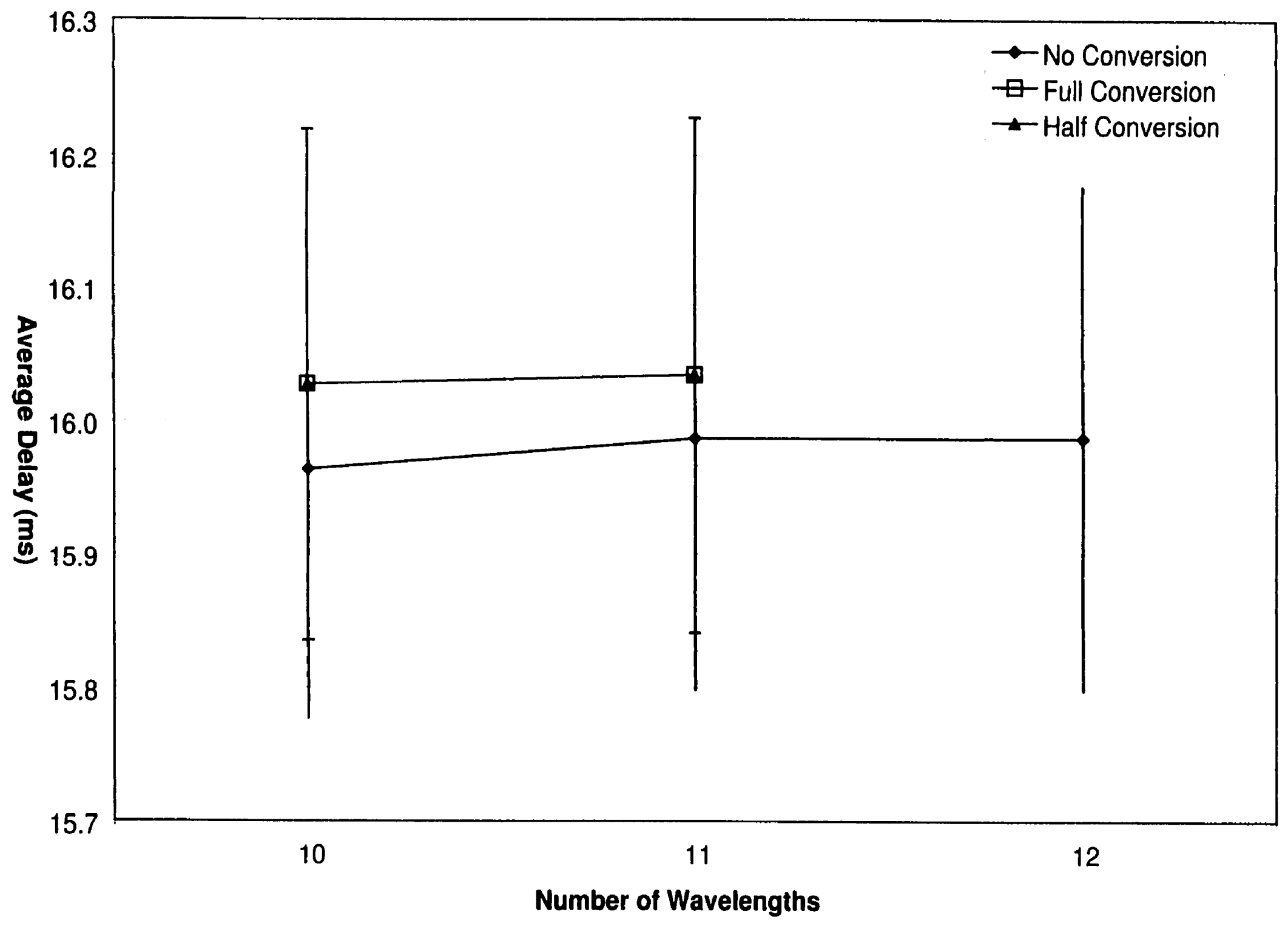

Figure 5-9: NSF / Uniform Traffic - Delay vs /W| for Policy 2 


$$
\pi \|
$$




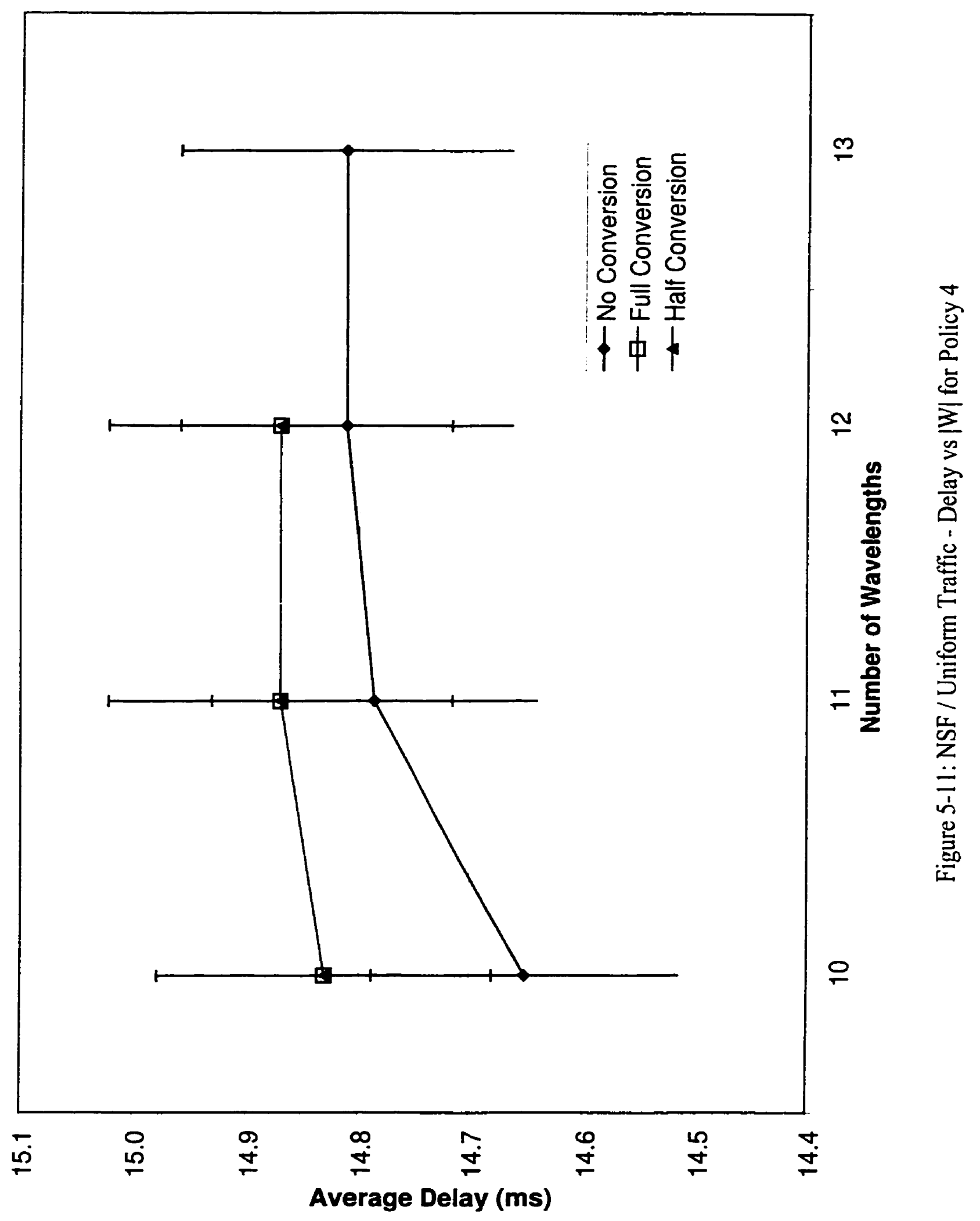




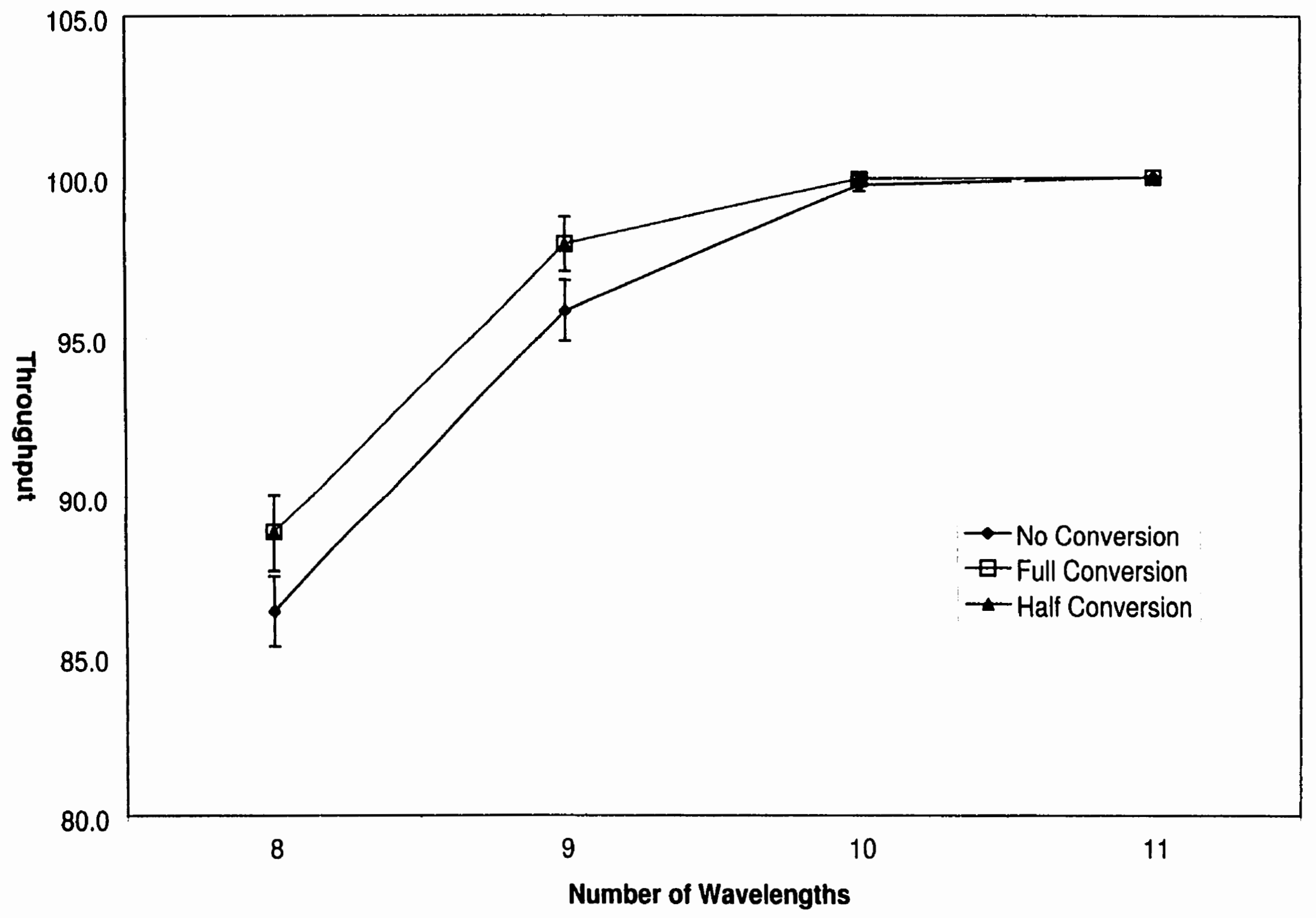

Figure 5-12: NSF / Non-Uniform Traffic - Throughput vs $|W|$ for Policy 1 


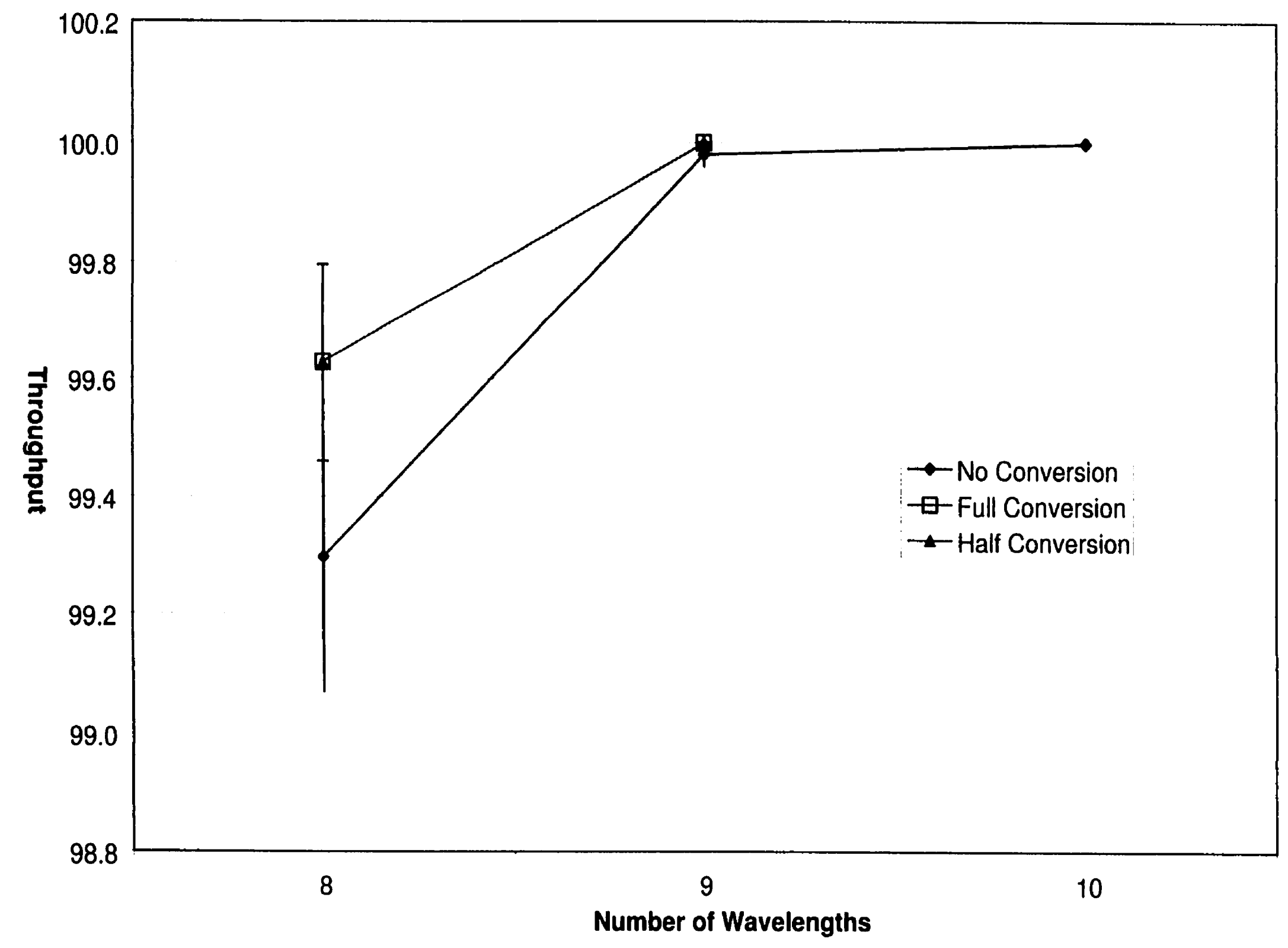

Figure 5-13: NSF / Non-Uniform Traffic - Throughput vs |W| for Policy 2 


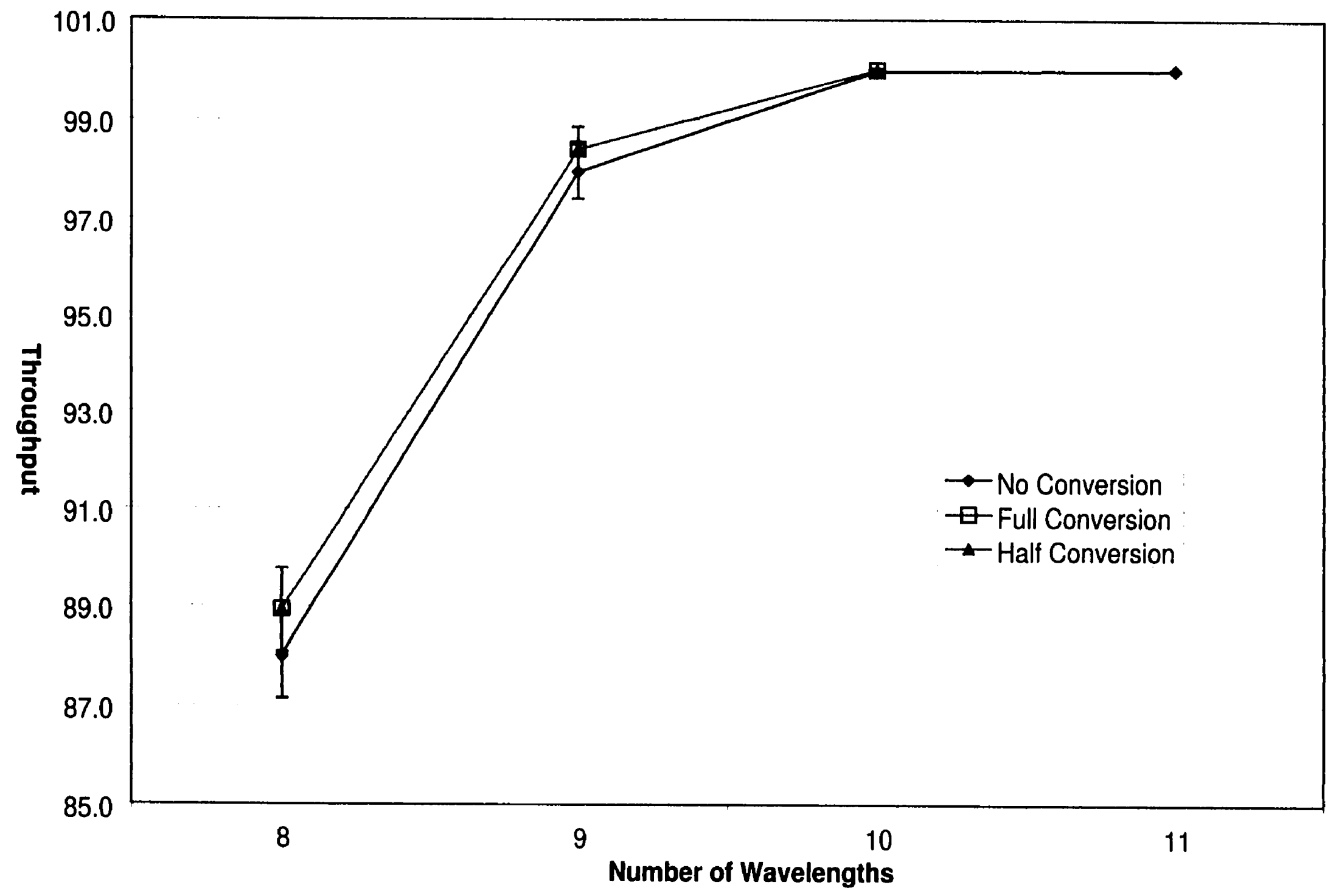

Figure 5-14: NSF / Non-Uniform Traffic - Throughput vs $\mid$ W| for Policy 3 


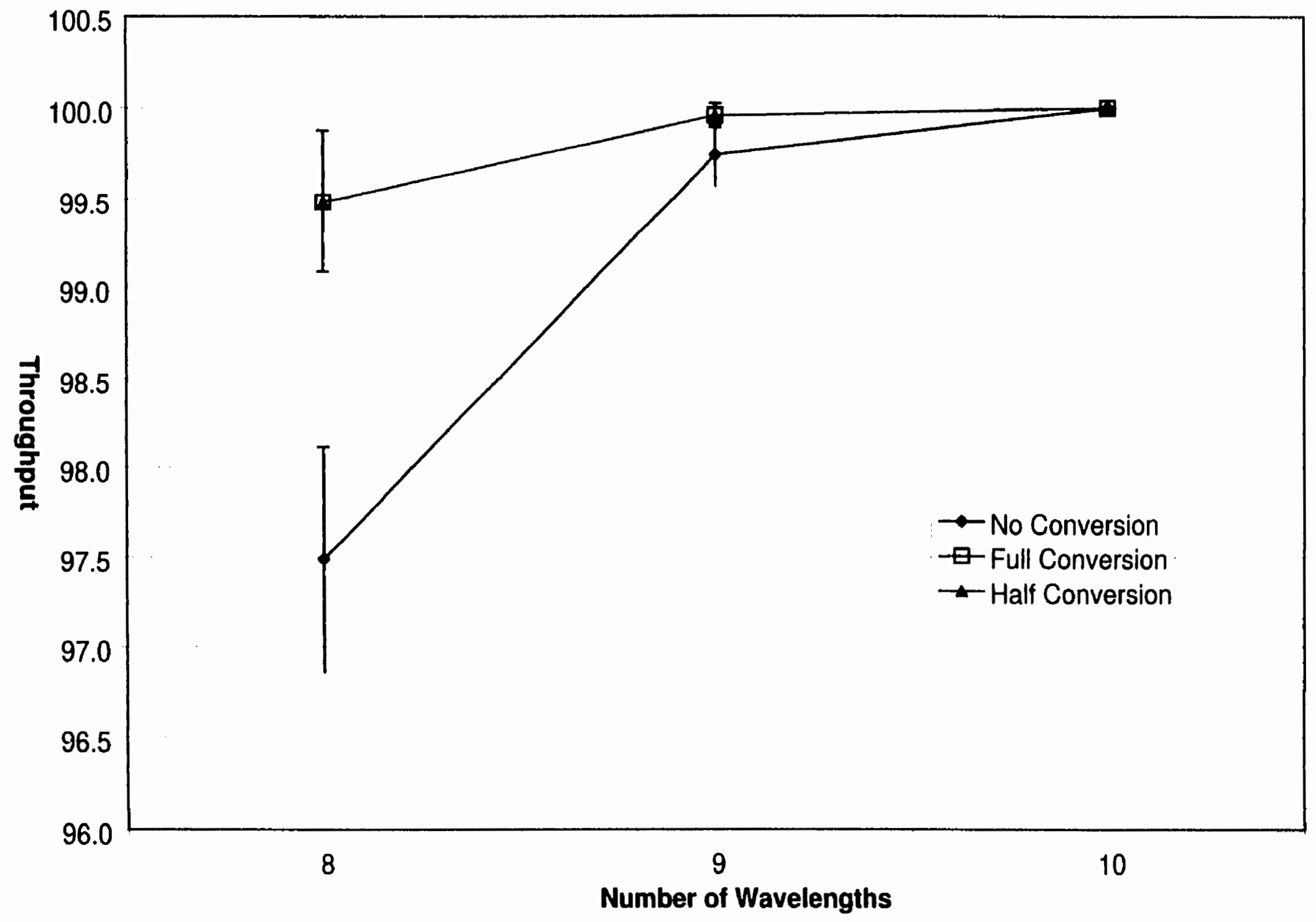

Figure 5-15: NSF / Non-Uniform Traffic - Throughput vs $|W|$ for Policy 4 


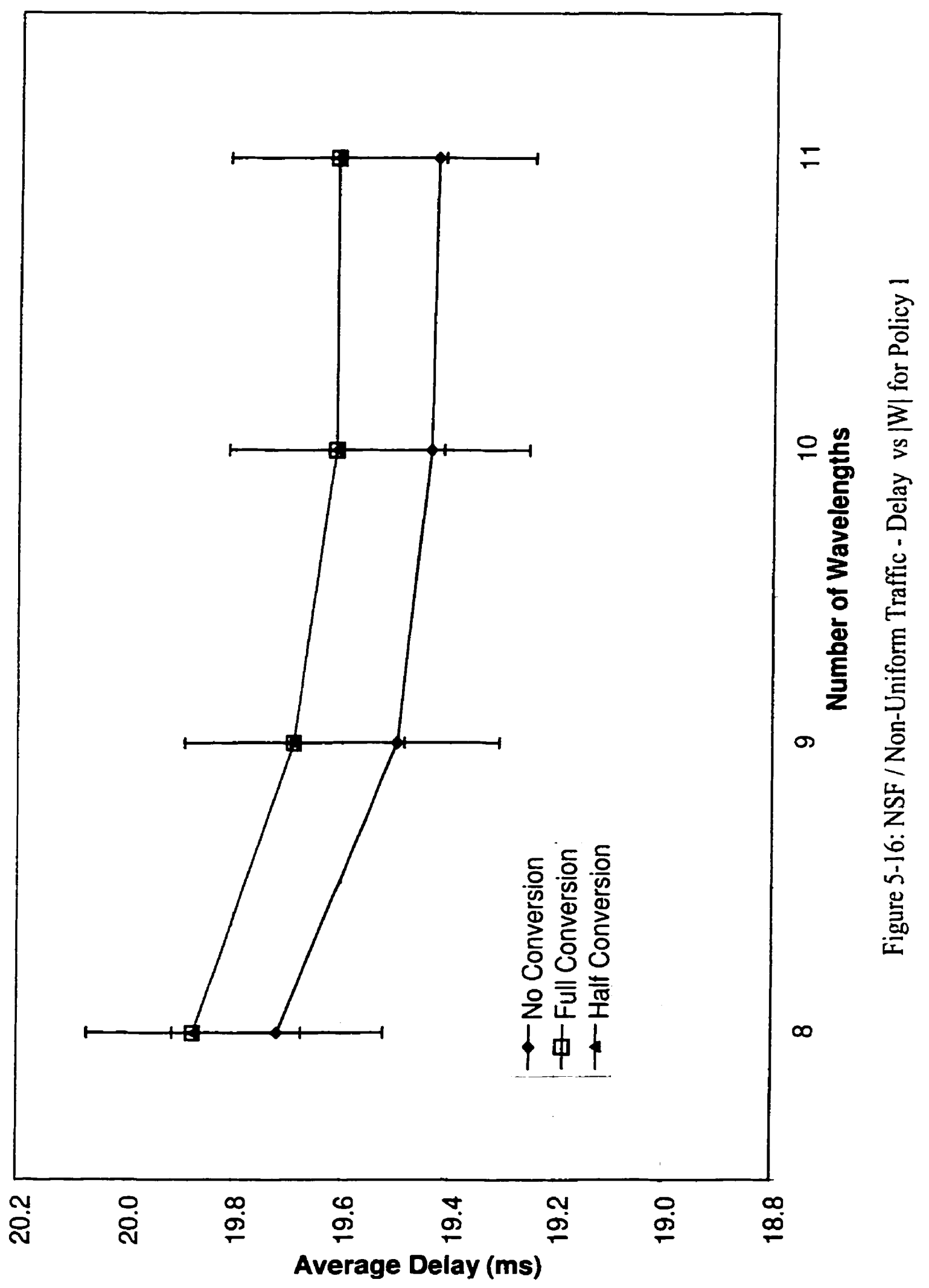




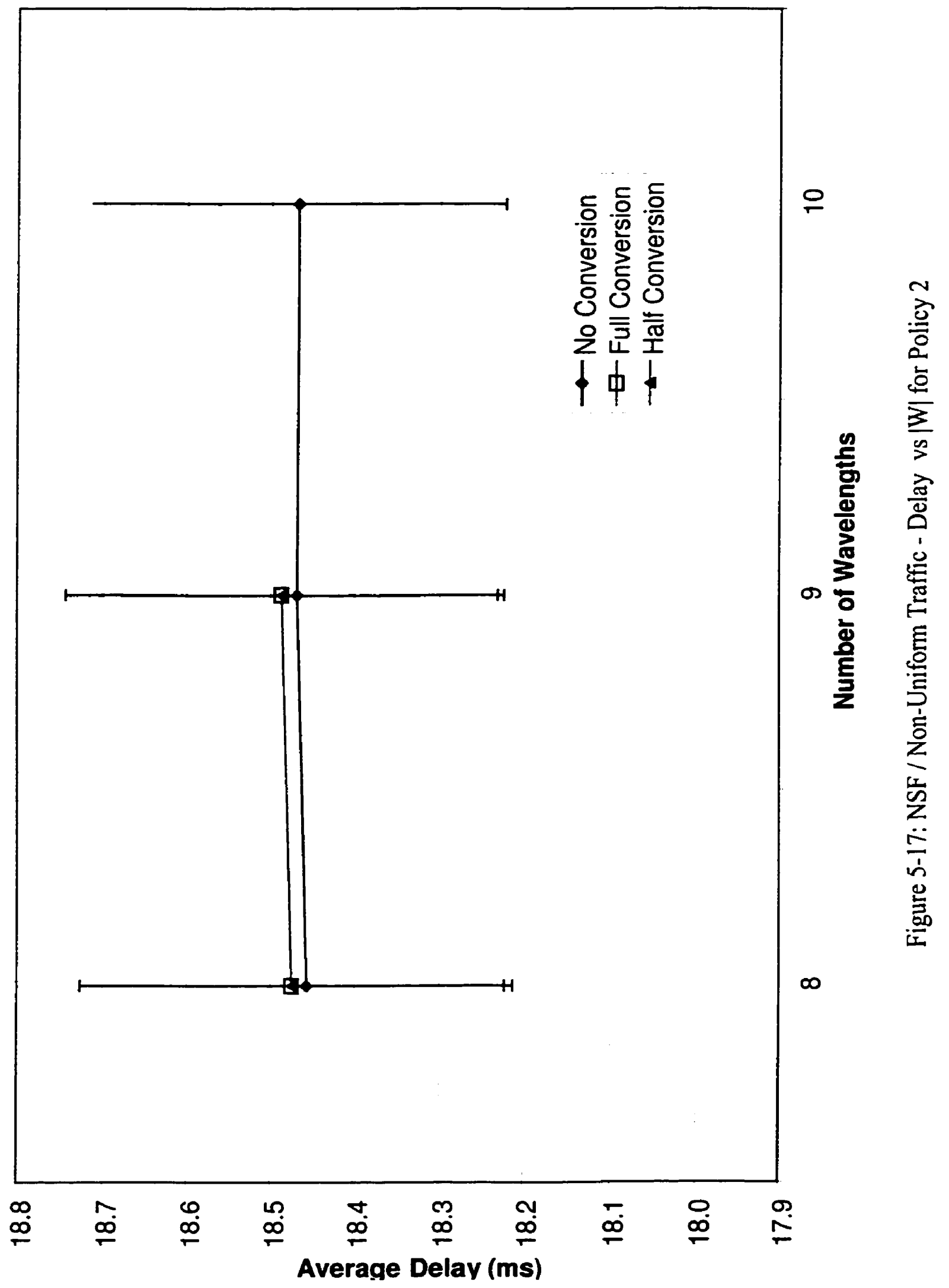




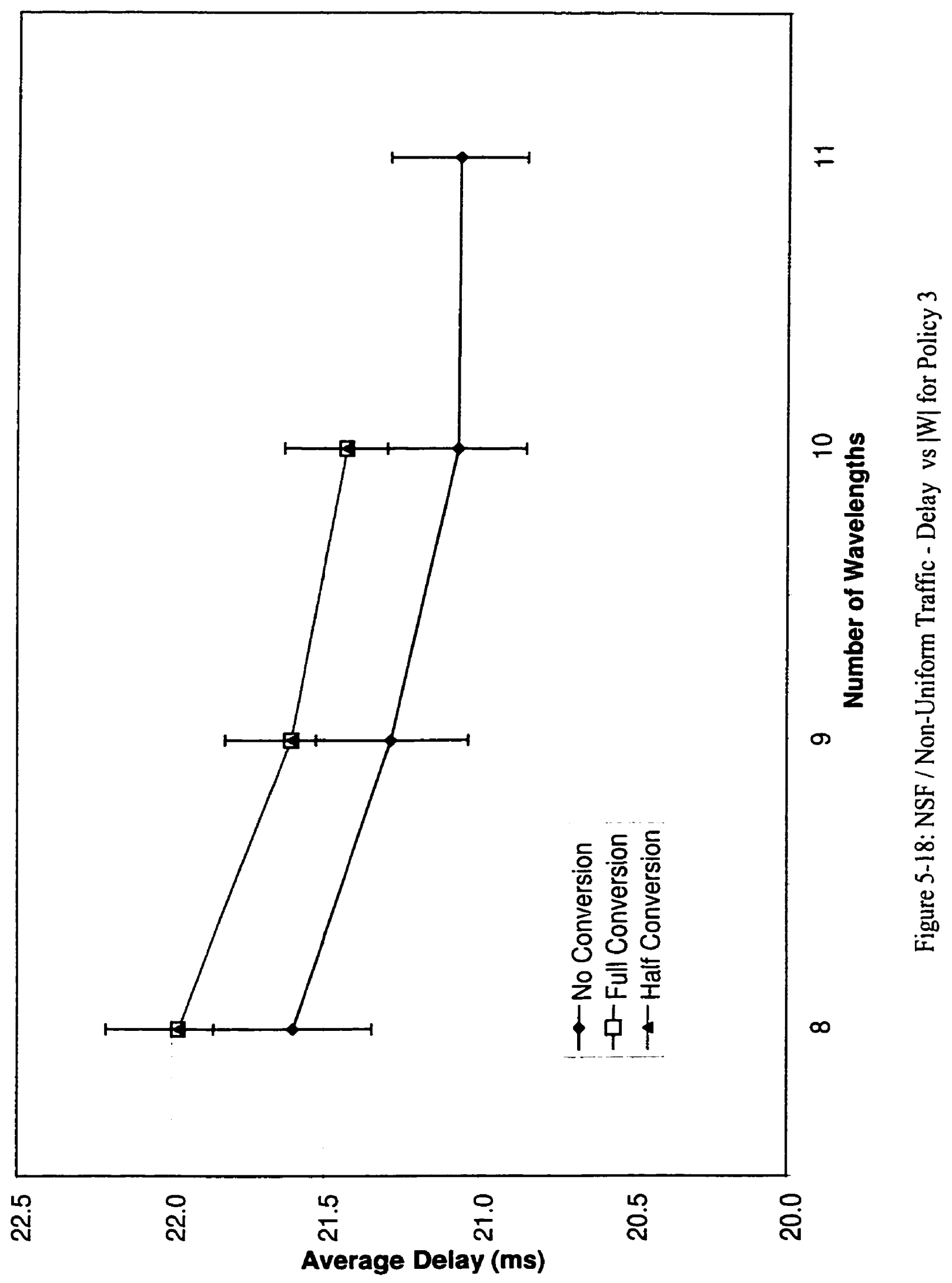




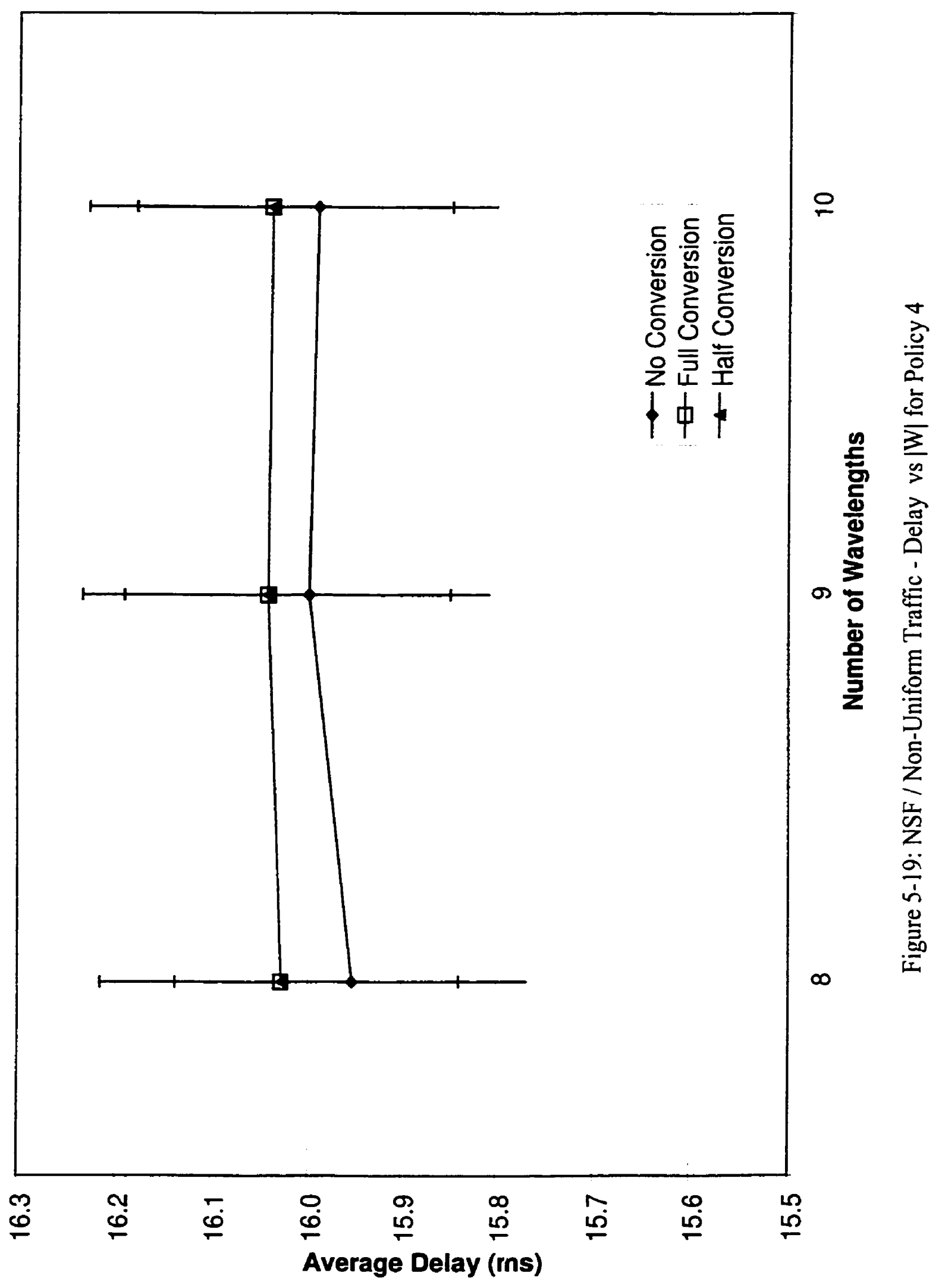




\subsection{Comparison of Switches in the NSF Network}

In the majority of cases, the use of wavelength converters increased the throughput for each $|W|$ ( Figure 5-4 - Figure 5-7 and Figure 5-12 - Figure 5-15). This is an expected result and intuitively makes sense. Allowing the use of wavelength converters at intermediate nodes increases the probability of finding a route from an arbitrary source to any destination. The use of wavelength converters also decreases the total number of wavelengths required to reach $100 \%$ throughput in most cases. This has a significant impact on the total network costs as shown in Figure 5-2 and Figure 5-3.

Routing policy 3 produced the only exception (see Figure 5-6). In this case, the use of wavelength converters did not produce any significant improvement. In this routing policy, the $s-d$ pairs with the longest physical distance are routed first. It is therefore more likely that the $s$ - $d$ pairs furthest apart will be routed using new continuous connections as they are attempted first before any other connections are made. As $|W|$ increases, only the $s$ - $d$ pairs with shorter physical paths are left to be routed, leaving very little leeway for the use of wavelength converters, since there is less of a need for a converter when the path is one or two physical hops.

When comparing average delay (Figure 5-8 - Figure 5-11 and Figure 5-16 Figure 5-19), the use of wavelength converters increases the average delay experienced by packets in the network. The simulator can find longer routes from the source to the destination when wavelength conversion is allowed at the intermediate nodes. 
The increasing trends seen in the delay figures is caused by averaging the total amount of traffic routed for each $\mid W$. As more $s-d$ pairs are routed, obviously more traffic is successfully routed. This increase in the total routed traffic is used in the calculation of the average, resulting in a higher average delay.

Routing policy 3 again produces the only exception (Figure 5-10 and Figure 518). In this routing policy, the $s-d$ pairs with the largest physical distance are routed first. Therefore, the average delay for the packets travelling between these nodes will be on the high end of the average (longer physical distance results in higher propagation delay). As the $s$-d pairs with a shorter physical distance are routed, the average delay will decrease as the average propagation delay decreases.

With a non-uniform traffic matrix, routing policy 1 also produces a trend of decreasing delay as $|W|$ increases. This goes against intuition, but can be explained as follows. With a value of $F=75 \%$, which was used in the non-uniform simulations, the majority of traffic in the matrix is uniformly and randomly distributed over the range $(0$, y) where $\mathrm{y}<\mathrm{x}$ (see section 4.4.2). Since $25 \%$ of the $s-d$ pairs are randomly and uniformly distributed over the range $(0, \mathrm{x})$, only a few $s-d$ pairs would have a large traffic value $(>y)$. On average, about $12 \%$ of the $s-d$ pairs have a traffic value larger than $y$. In routing policy 1 , the $s$ - $d$ pairs are sorted in increasing order of their traffic demands. The few $s-d$ pairs with large traffic demands are not routed until the end. At this point, there is a high probability that the lower wavelengths are full because the wavelengths are reused as much as possible to utilize the entire available bandwidth. As such, there is a high probability that the s-d pairs with higher traffic demands are routed using a new 
continuous connection. This being the case, these new connections would obviously take the shortest possible route, resulting in a lower propagation delay. With the majority of the traffic experiencing lower propagation delay, the average delay would be brought down as more traffic is routed.

In all cases, the half-clear wavelength convertible switch (labeled as half conversion in the figures) performs the same as the dedicated wavelength convertible switch (labeled full conversion in the figures). This indicates that the half-clear wavelength convertible switch is a cheaper alternative to the dedicated wavelength convertible switch without compromising performance. Costs associated with the halfclear wavelength convertible switch are only slightly higher than the costs of the network without wavelength converters. Due to the fact that fewer wavelengths are required to route $100 \%$ of the traffic, it is actually cheaper to use the half-clear wavelength convertible switch than no conversion (Figure 5-2 and Figure 5-3).

\subsection{The European Optical Network}

In this section, the EON topology was used as the base for comparison. The following input-data was used for this investigation:

- Physical Topology: The 19-node EON as shown in Figure 5-20 [31]. The distances (in $\mathrm{km}$ ) of the physical links between the nodes are given in the following matrix: 


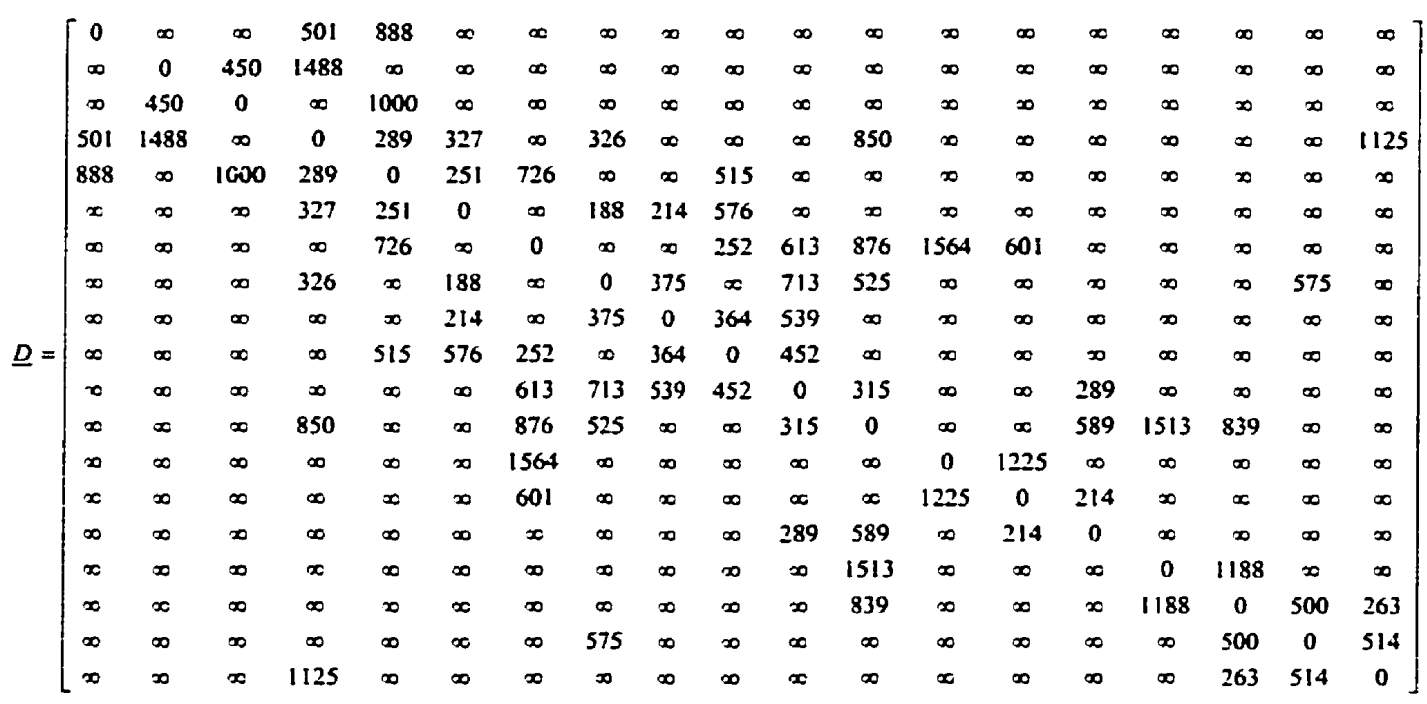

- Traffic: both uniform and non-uniform traffic was considered.

- Node Architecture: No wavelength conversion, full conversion and half conversion (the half-clear wavelength convertible switch) were considered.

- A time delay constrain of $30 \mathrm{~ms}$ was imposed.

- No constraint on the virtual link capacity utilization was imposed.

Results for this network can be found in Figure 5-21 - Figure 5-38. In Figure 5-21 and Figure $5-22$, the $x$-axis is labeled with the routing scheme used, where the first number refers to the routing policy (1-4) and the second letter refers to full $(F)$, no $(N)$ or half $(H)$ conversion. 


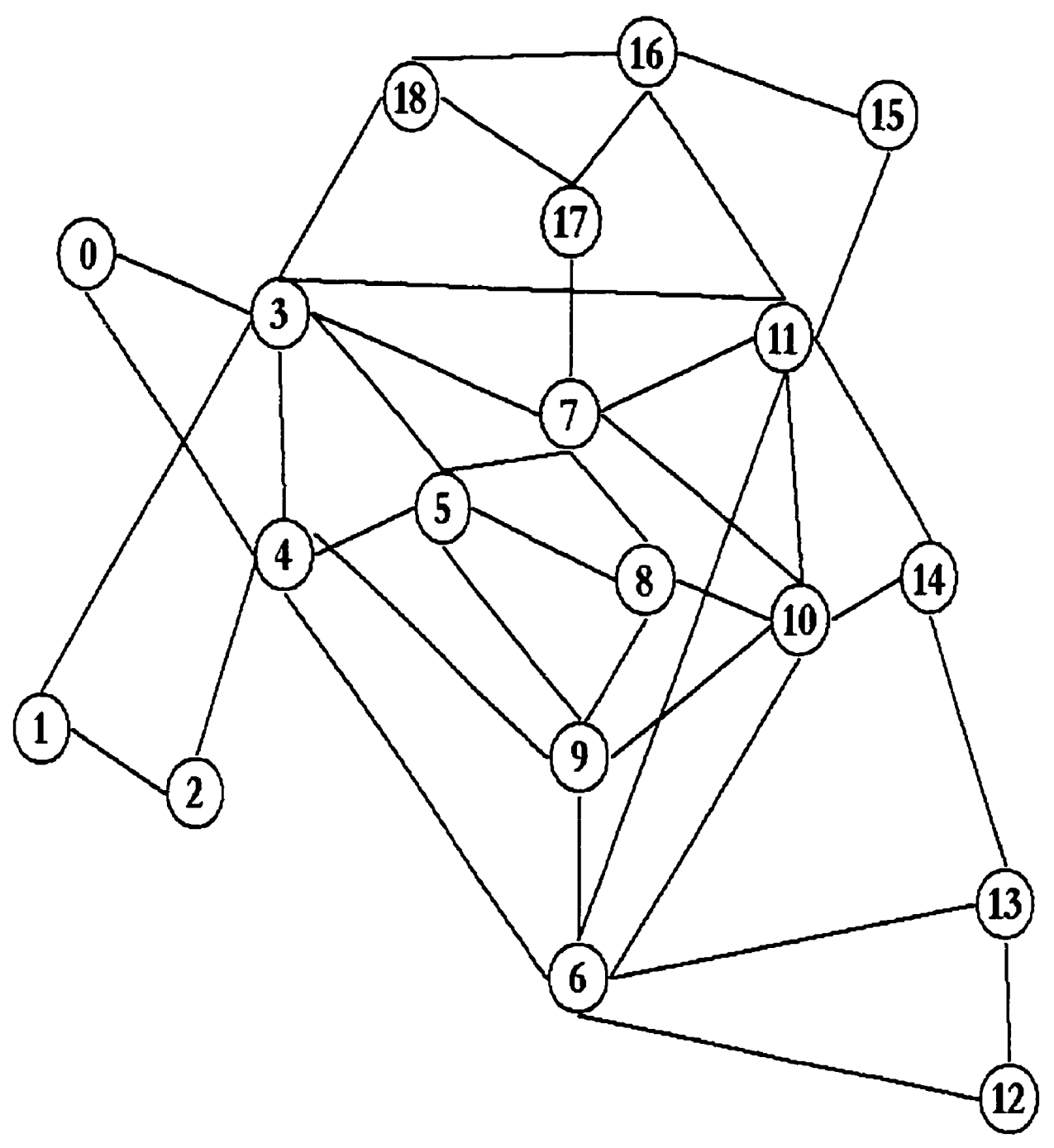

Figure 5-20: The 19-node EON wide-area network 

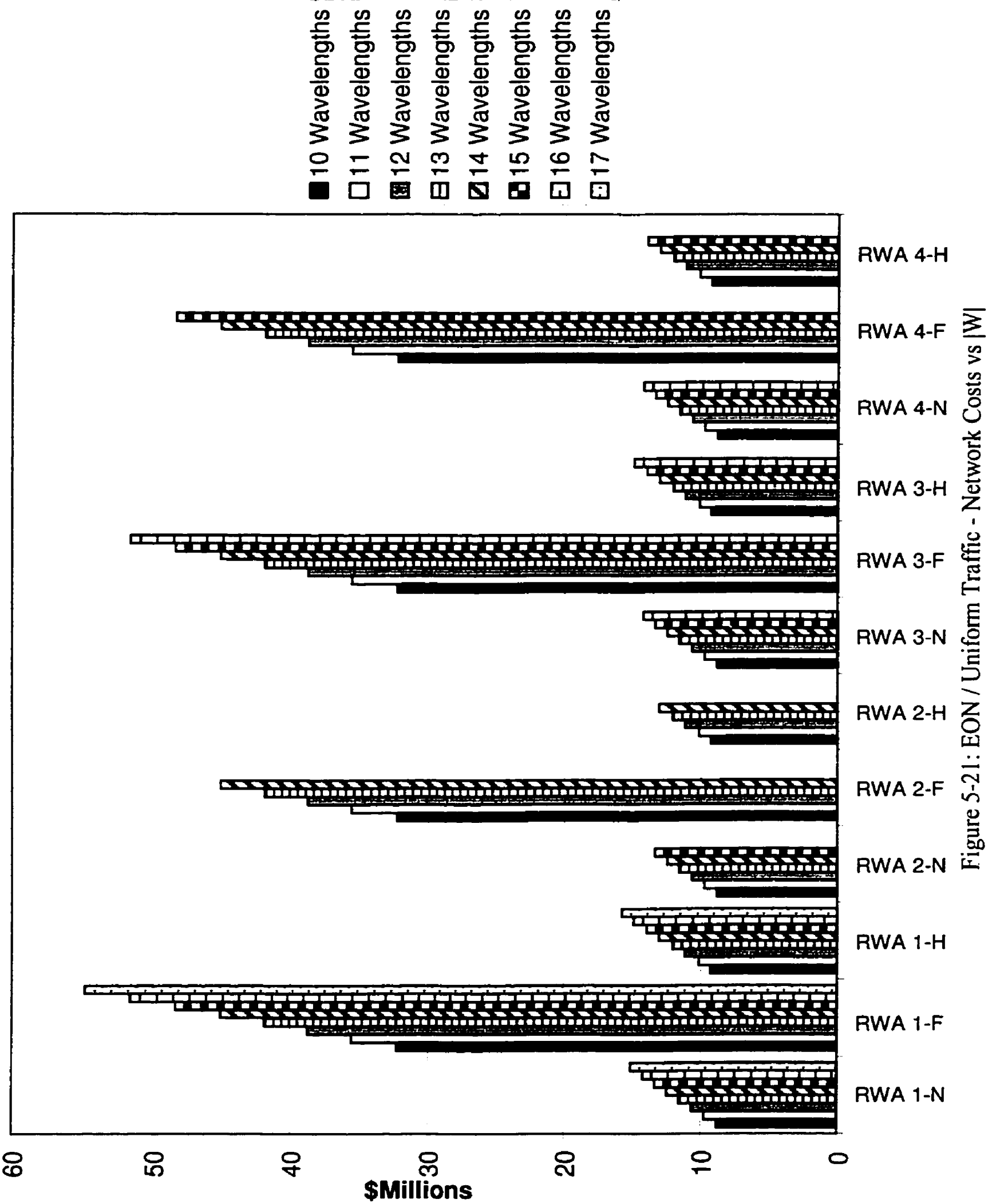

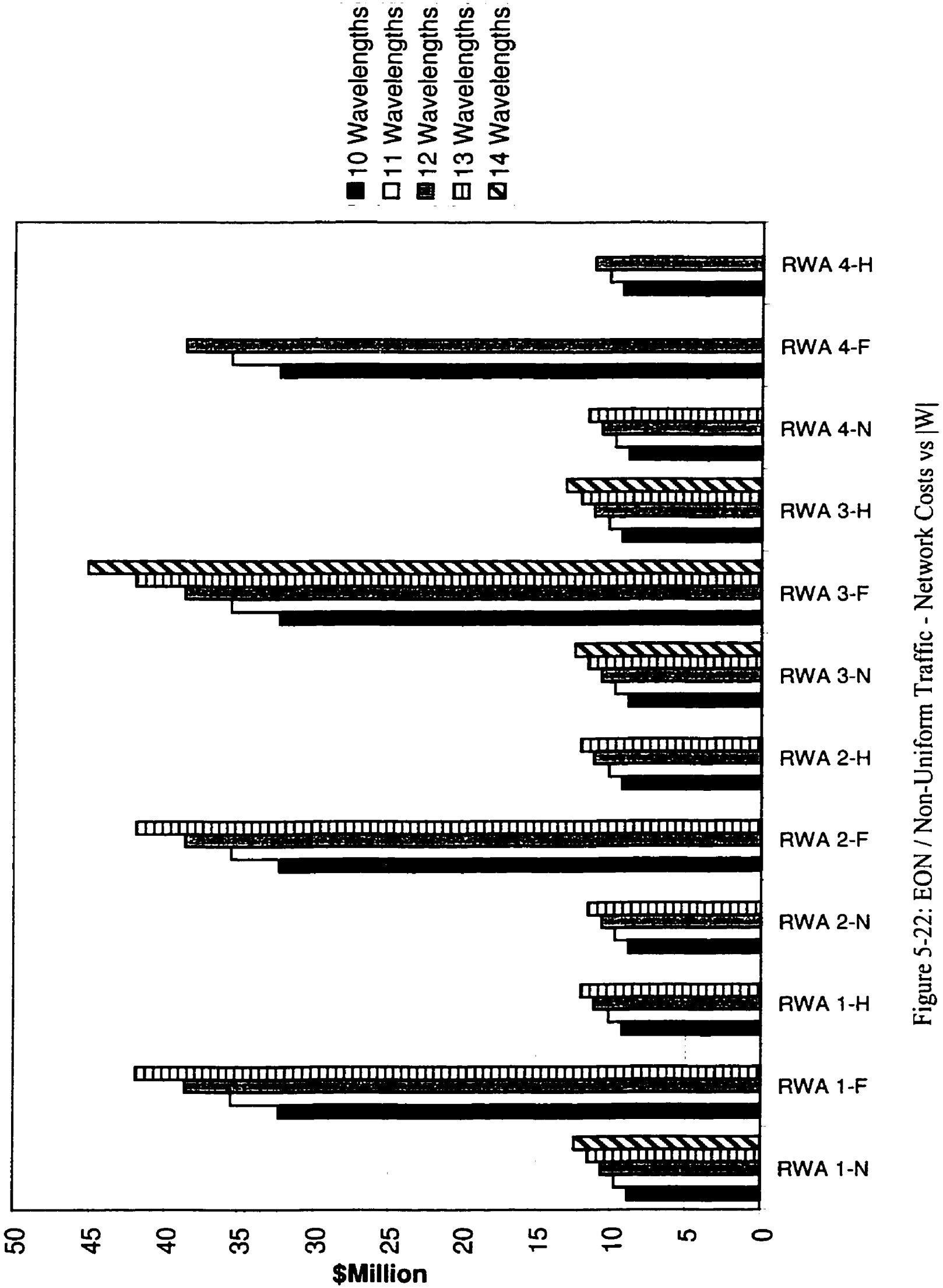


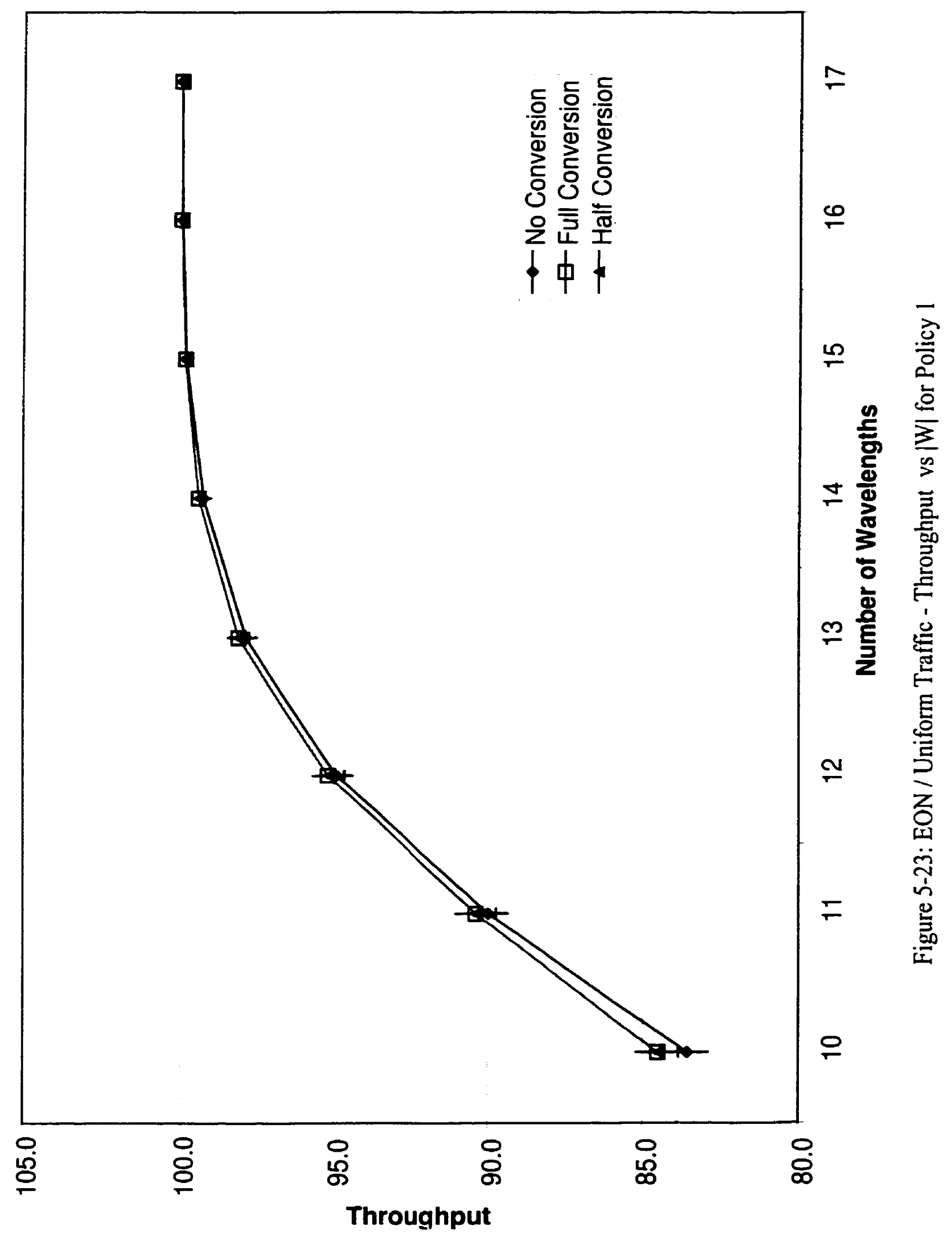




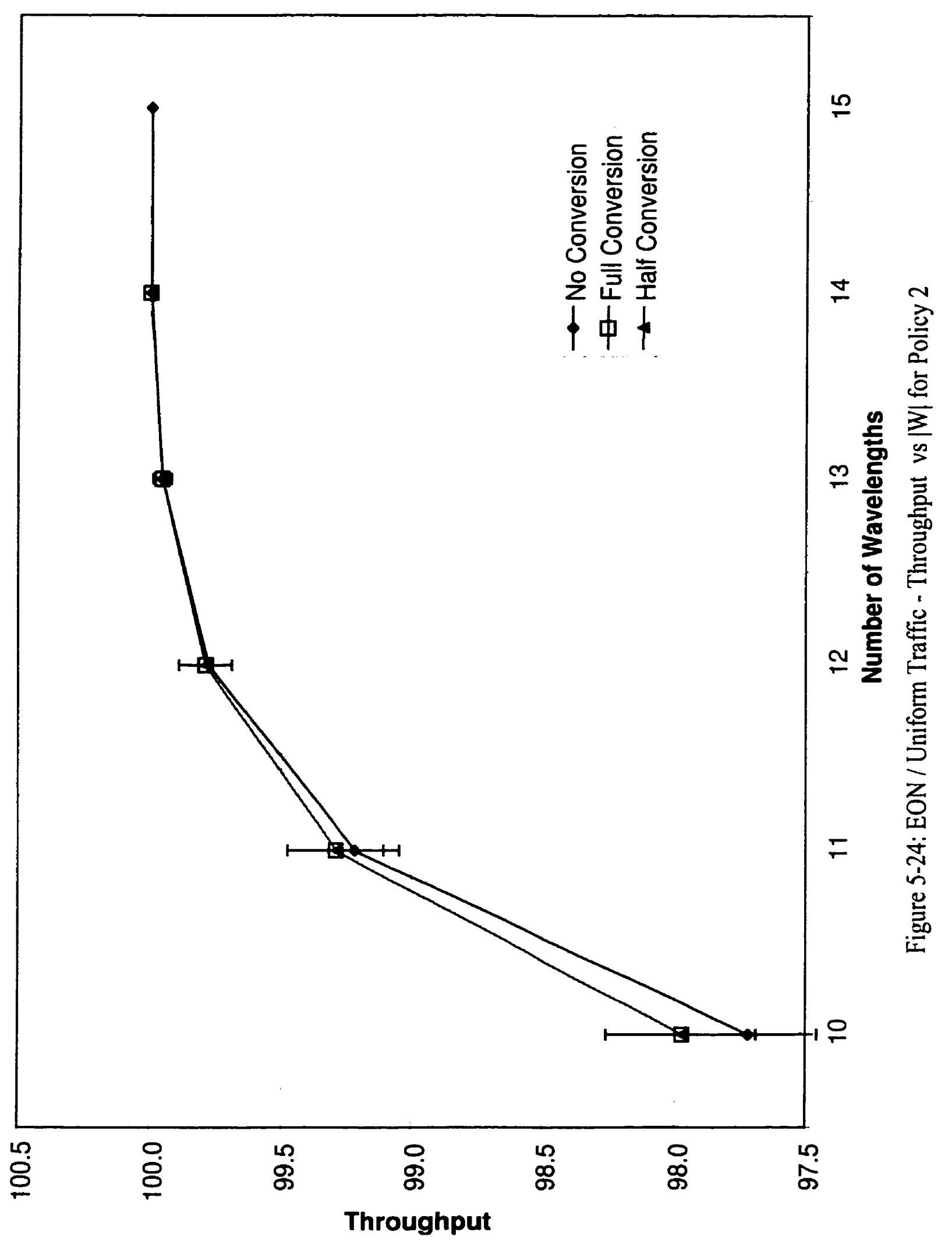




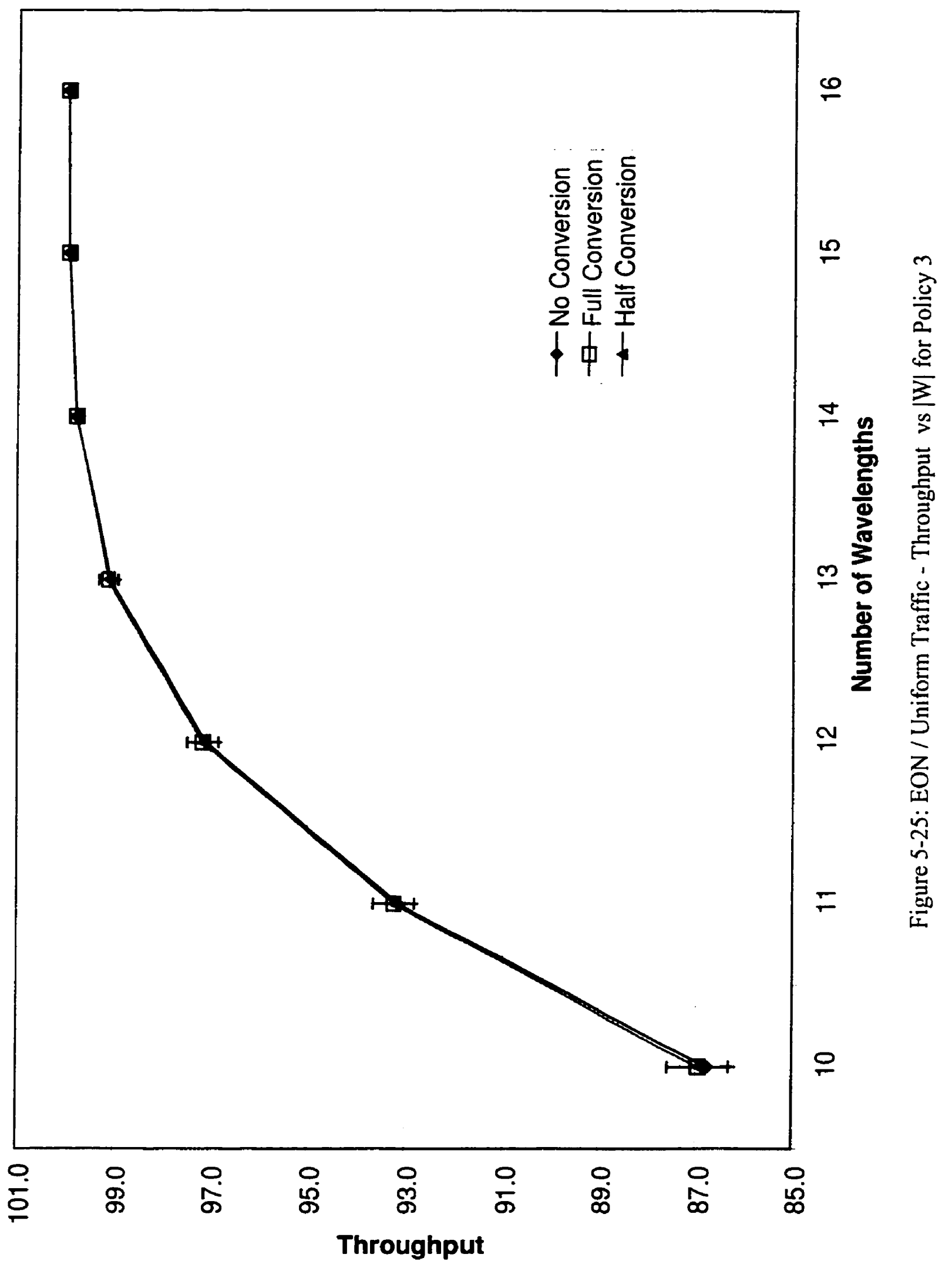




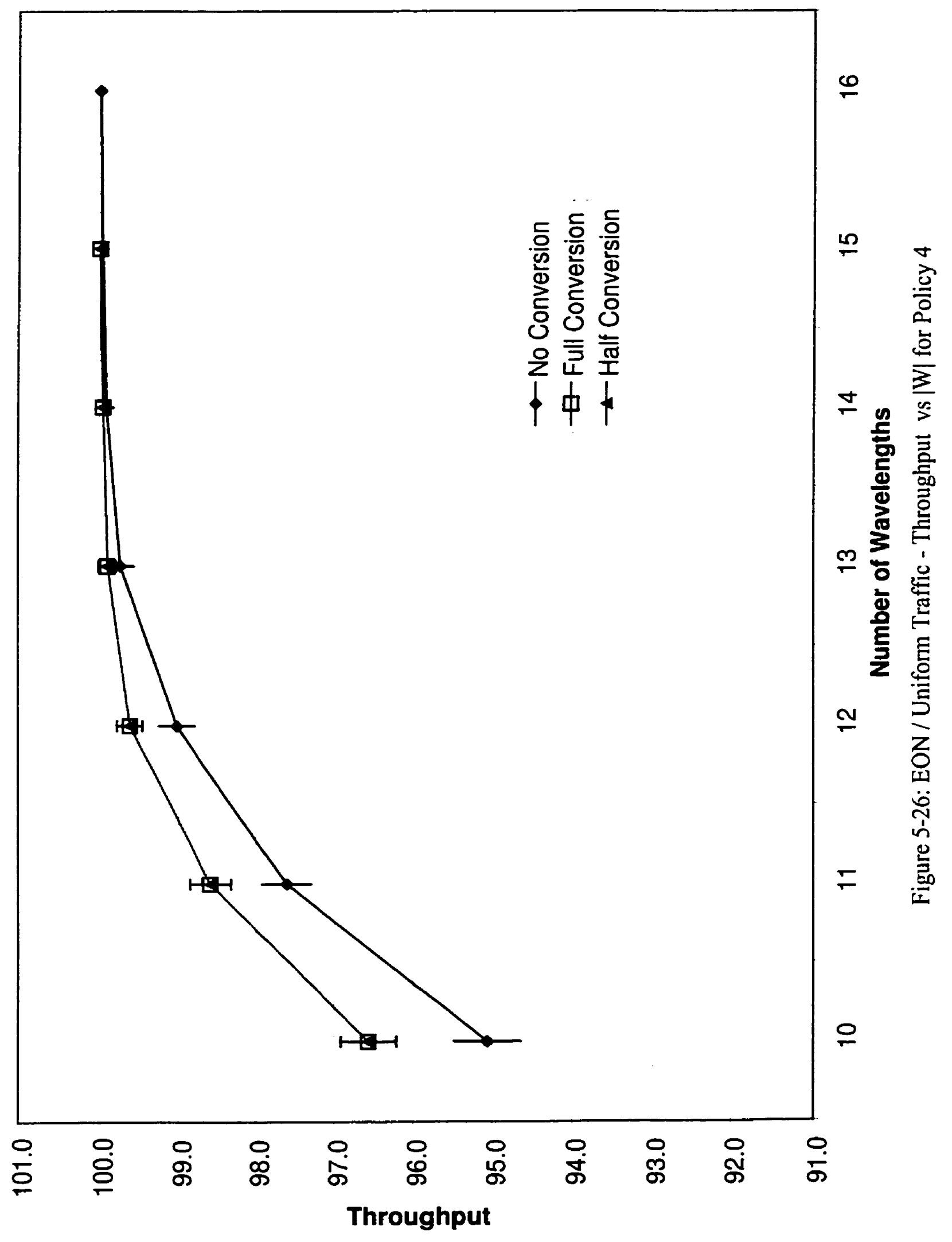




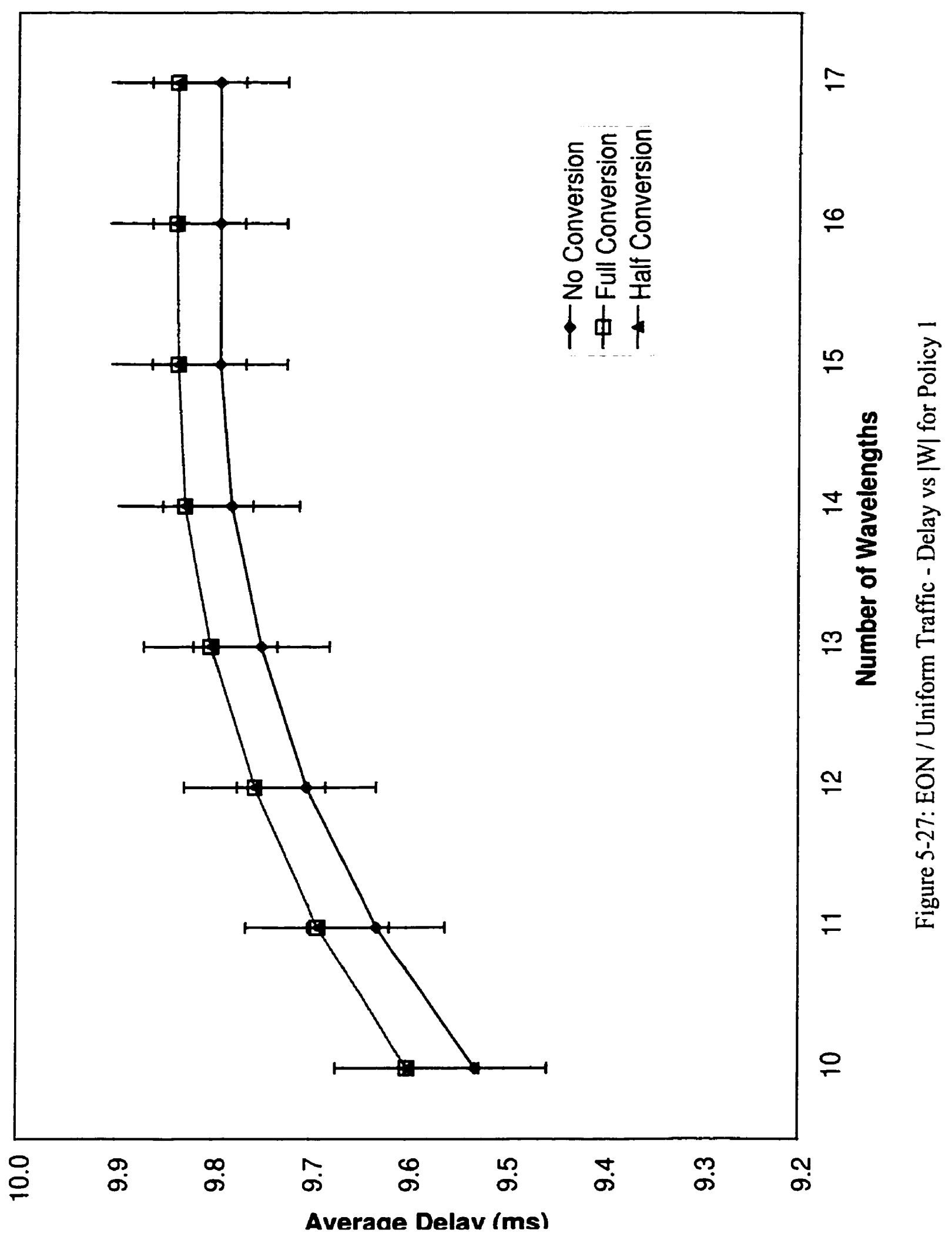




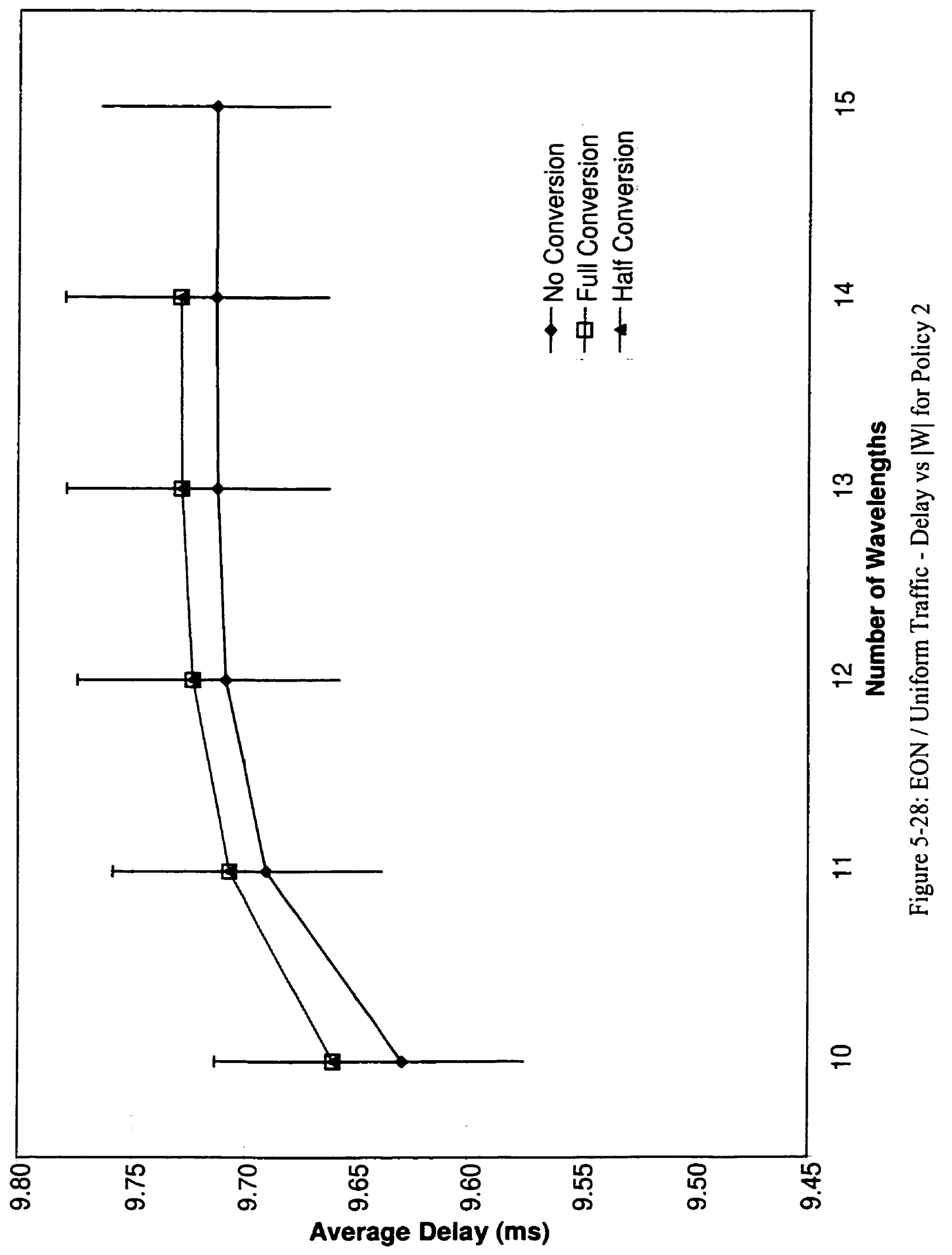




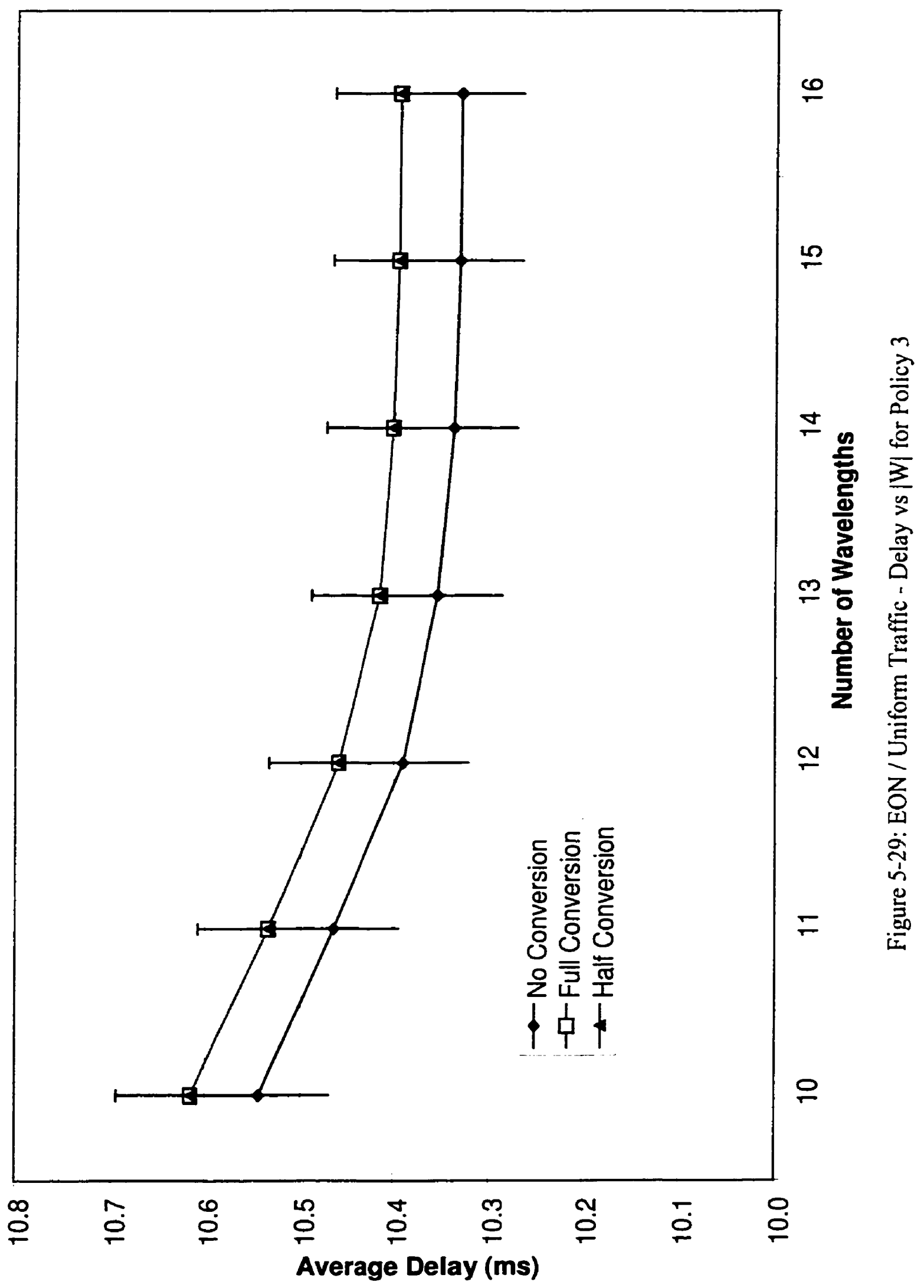




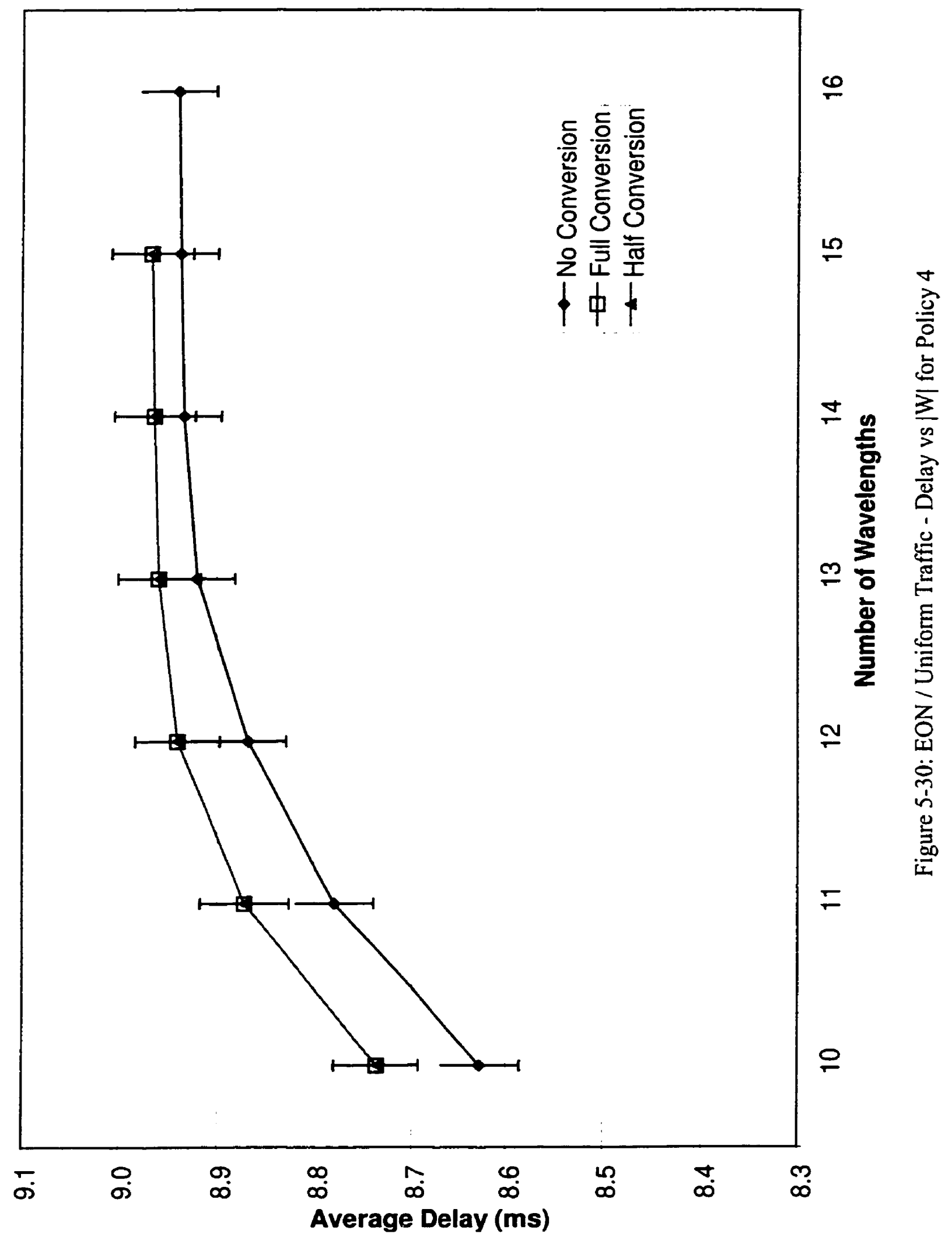




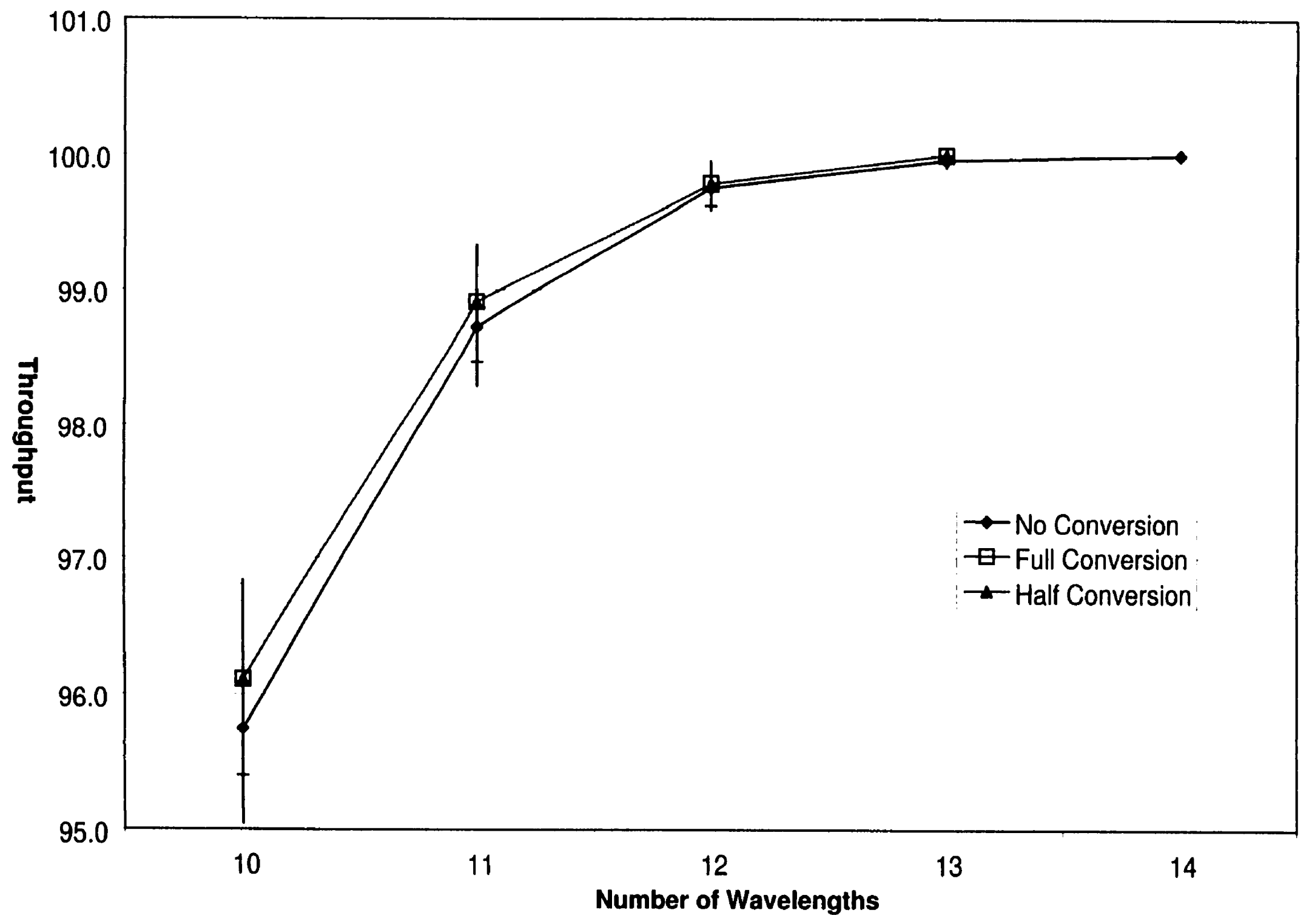

Figure 5-31: EON / Non-Uniform Traffic - Throughput vs $|W|$ for Policy 1 


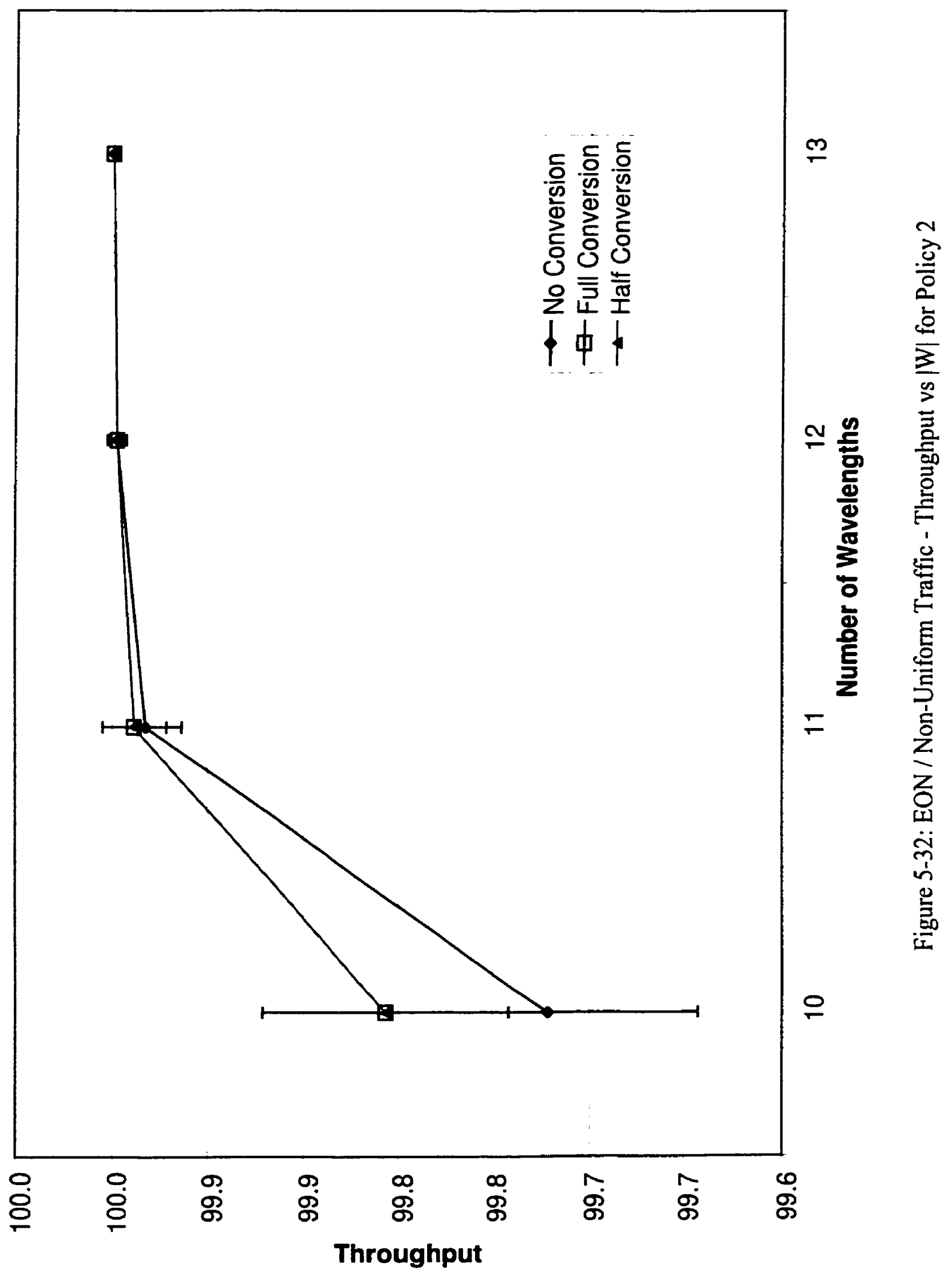




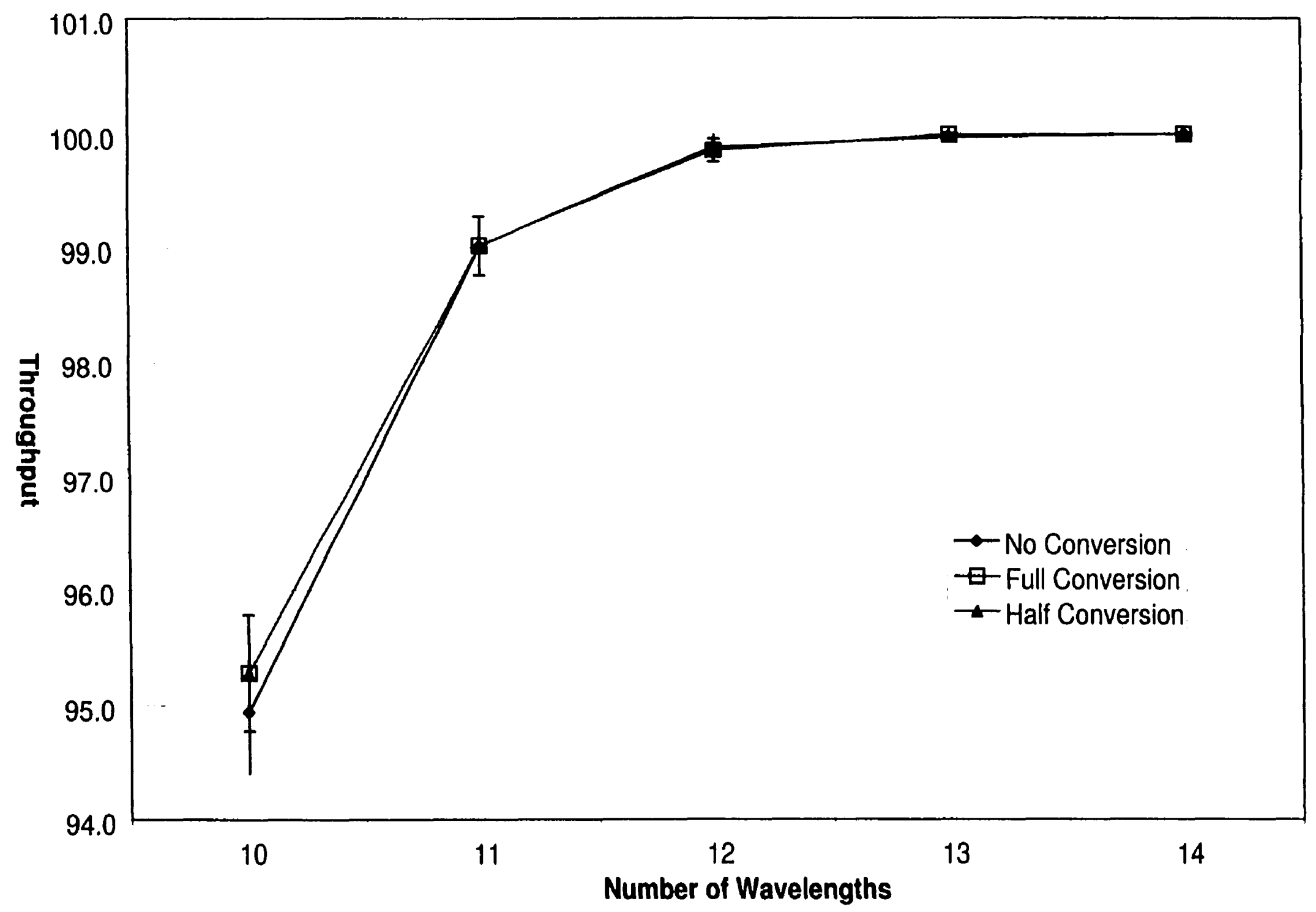

Figure 5-33: EON / Non-Uniform Traffic - Throughput vs $|W|$ for Policy 3 


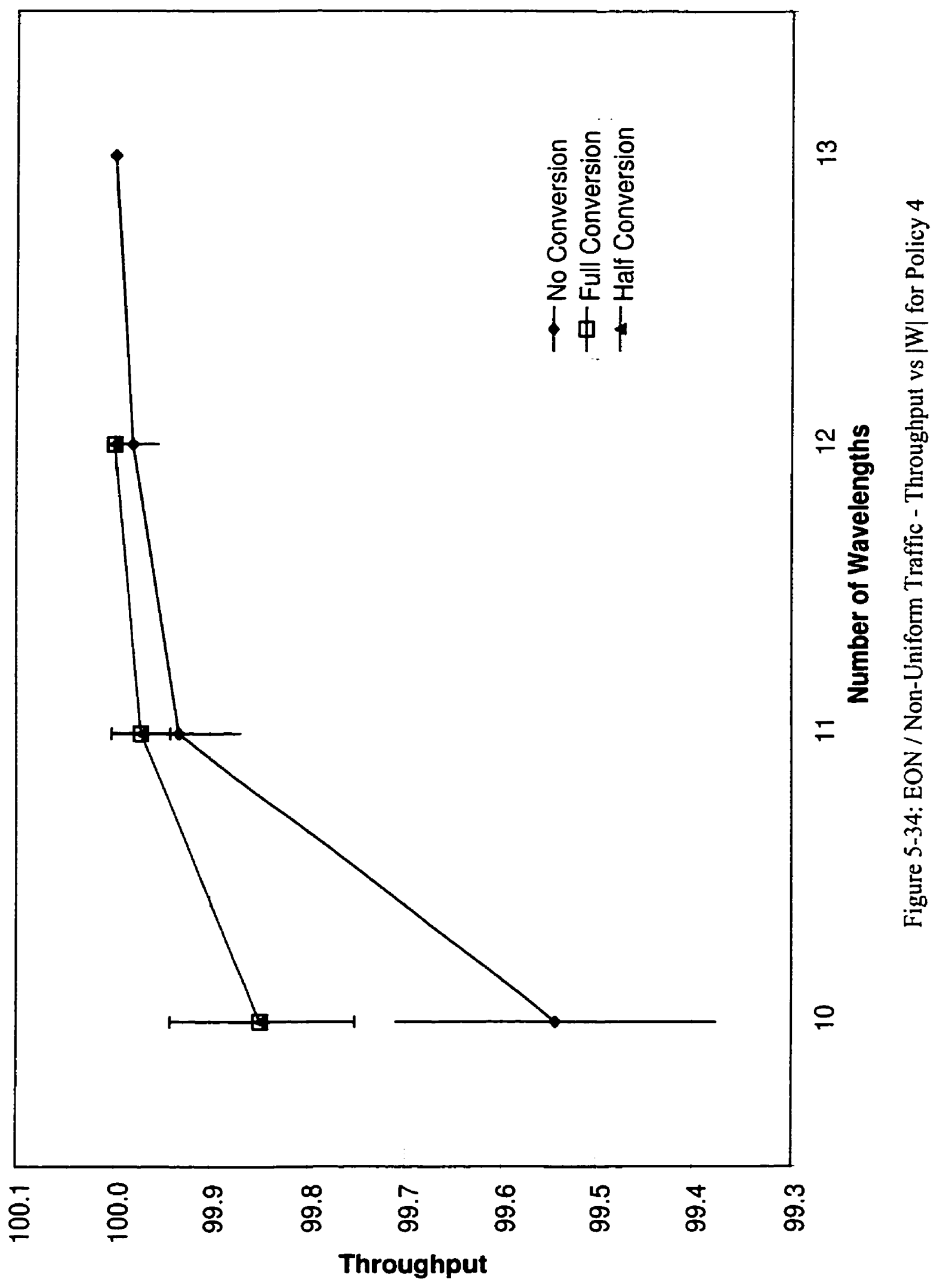




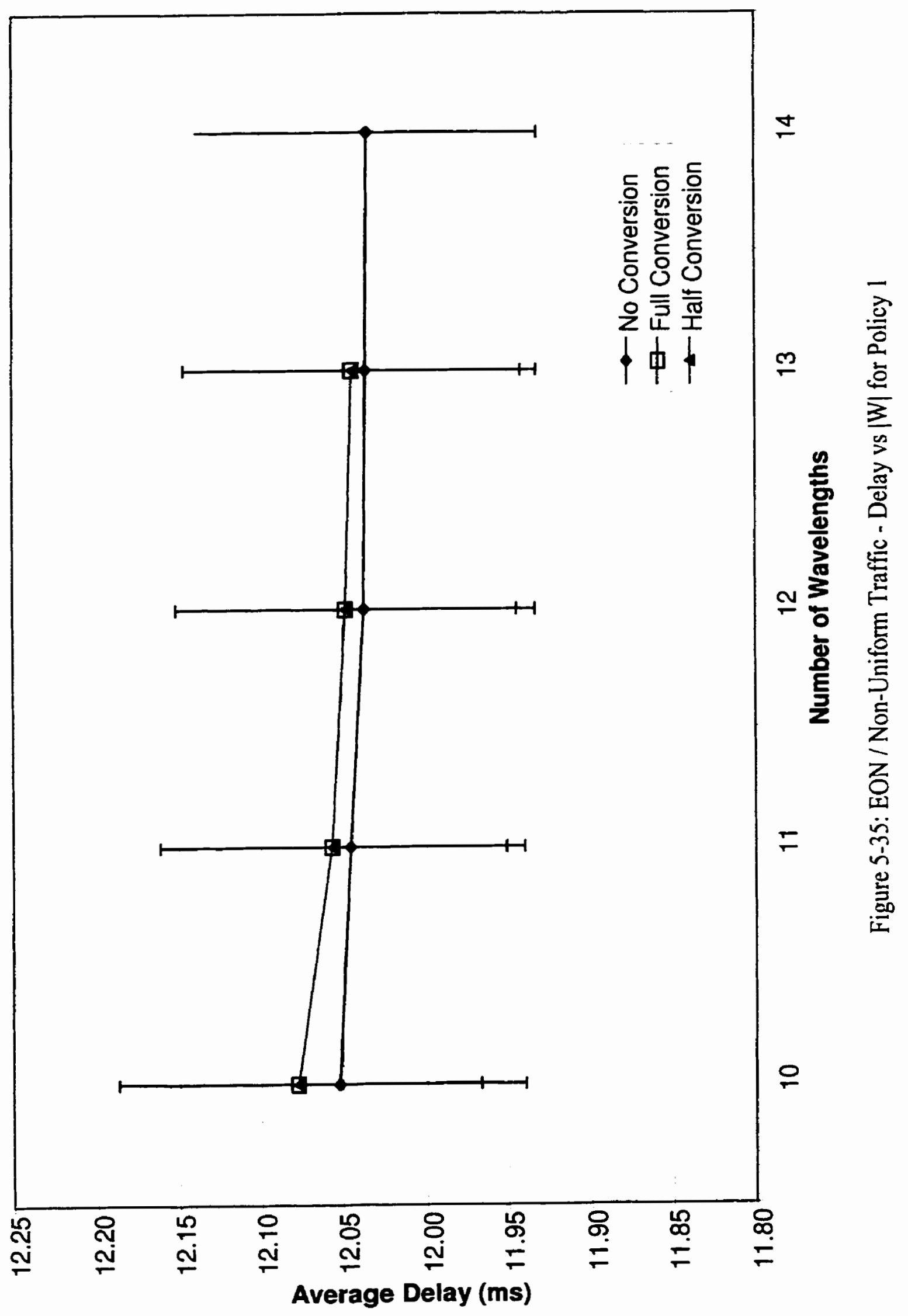




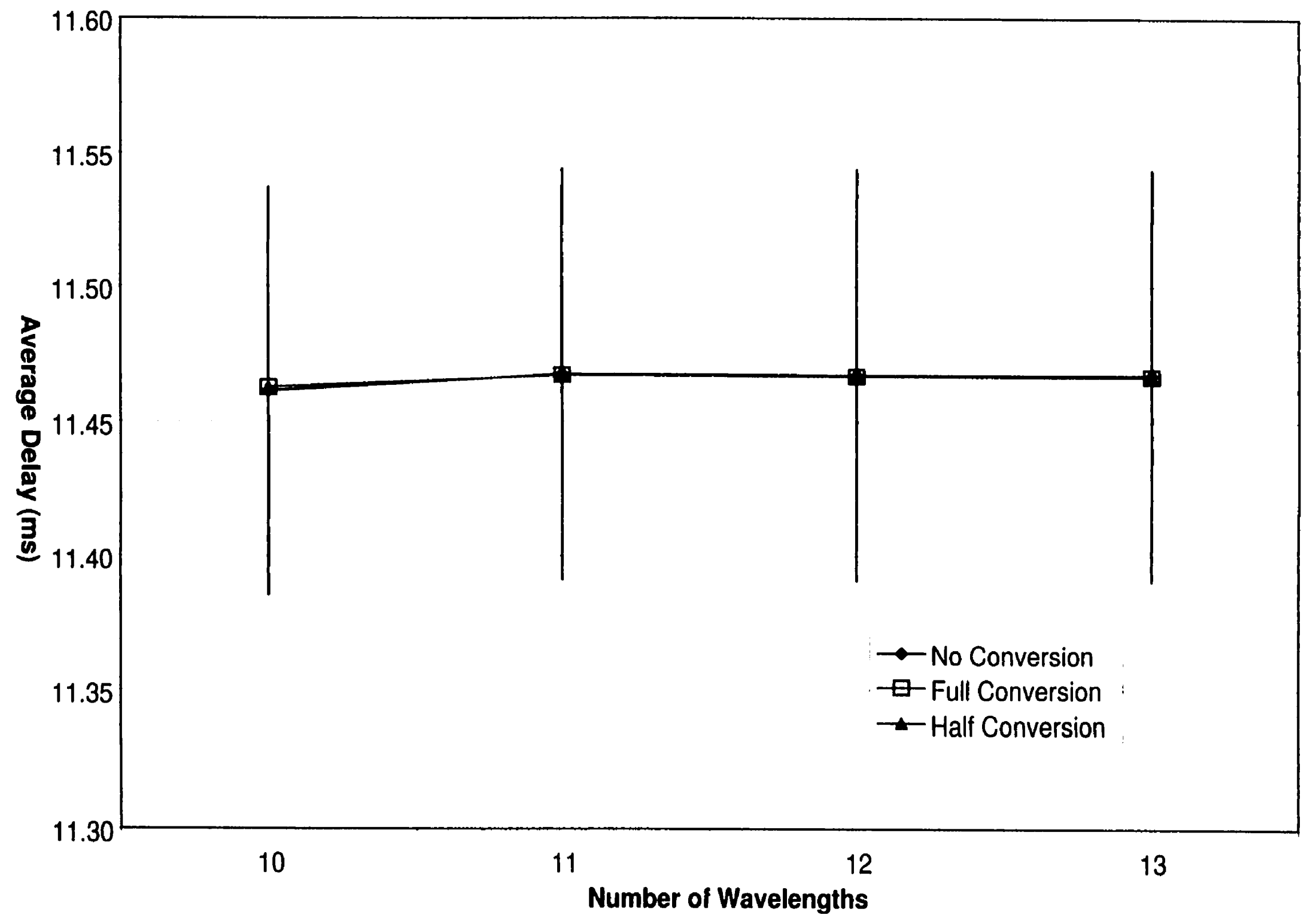

Figure 5-36: EON / Non-Uniform Traffic - Delay vs |W| for Policy 2 


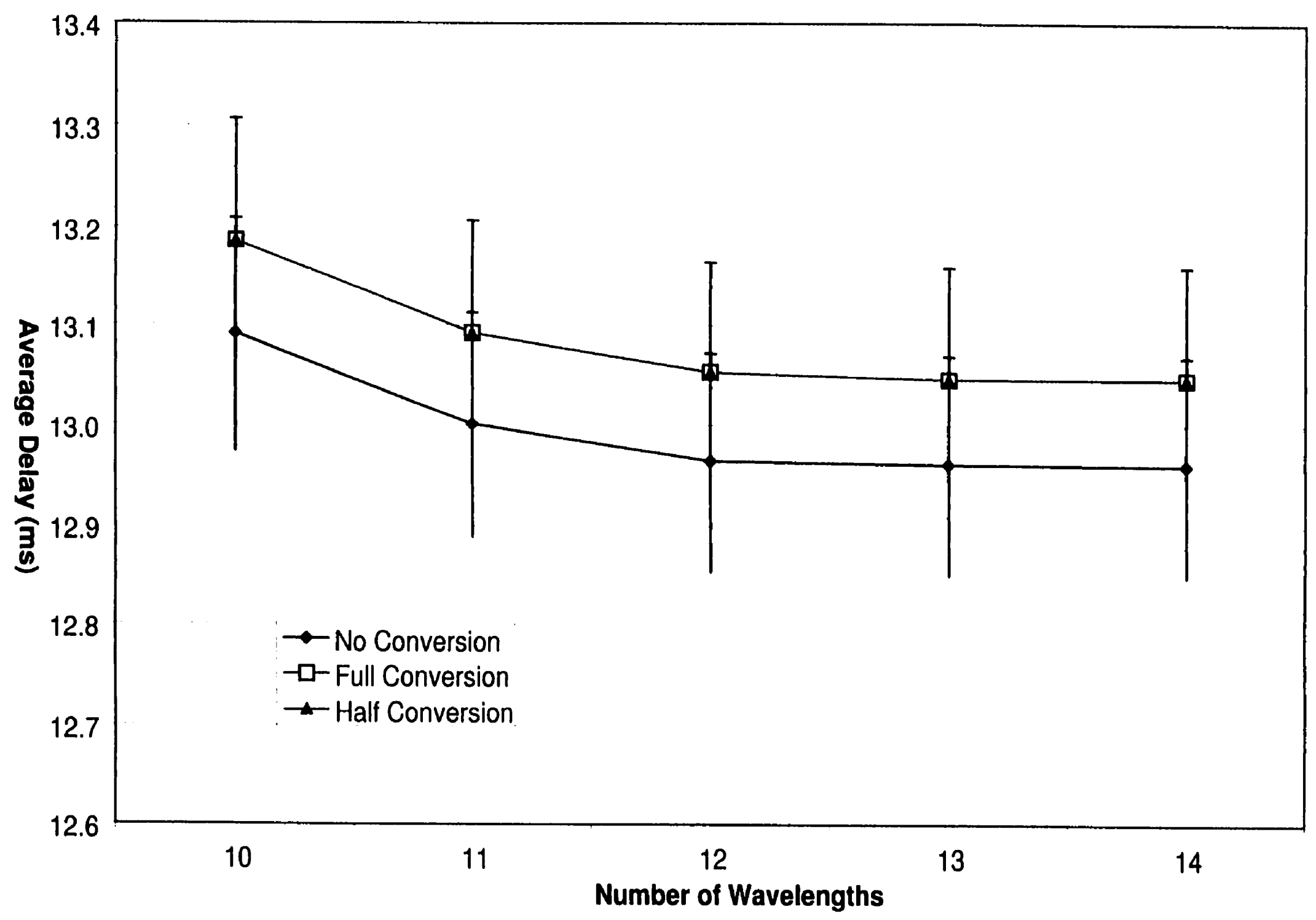

Figure 5-37: EON / Non-Uniform Traffic - Delay vs |W| for Policy 3 


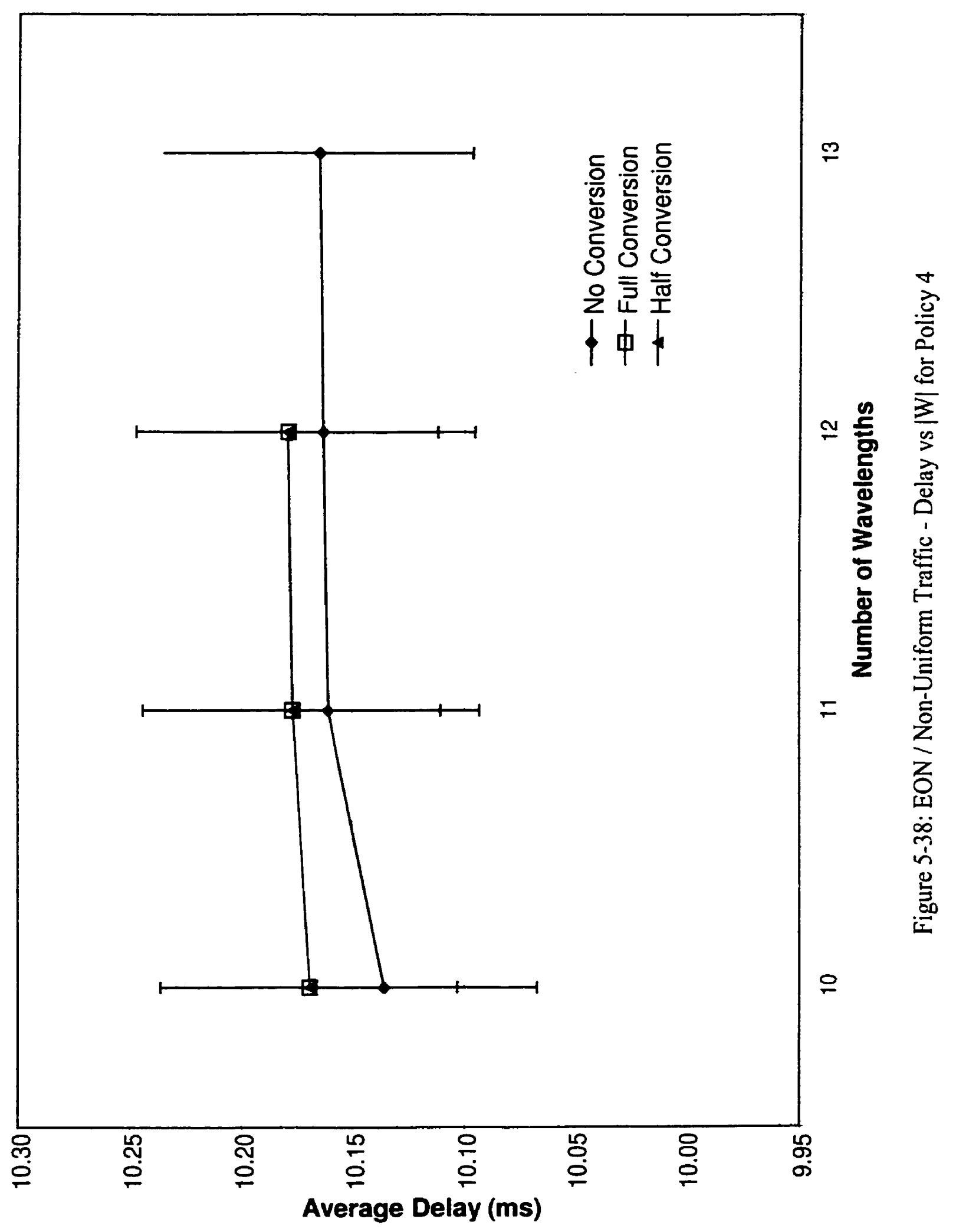




\subsection{Comparison of Switches in the EON}

As shown in Figure 5-21 to Figure 5-38, a number of simulations were run using 19-node European Optical Network. Uniform and non-uniform traffic matrices were used in each of the four routing policies to compare the performance of the half-clear wavelength convertible switch with the dedicated wavelength convertible switch (full conversion) and with no conversion. An average of 50 independent simulation runs was used.

The results for the EON are similar to that of the NSF Network discussed in the previous section. Again, for the majority of cases, the use of wavelength converters increased the throughput, and increased the delay for each $|W|$. The difference between the use of converters and no conversion is not as much in the EON however, due to the shorter distances and increased connectivity within the EON.

In the majority of cases, fewer wavelengths are required to reach $100 \%$ throughput using wavelength converters. This has a significant impact on the total network costs as depicted in Figure 5-21 and Figure 5-22. Trends seen in these figures follow the same trends as those of the NSF Network, and are explained in the previous section.

As in the NSF Network, the half-clear wavelength convertible switch proved to be a good, cost effective alternative to the dedicated wavelength convertible switch. 


\subsection{Comparison of Uniform vs Non-Uniform Traffic}

When comparing the uniform traffic case against the non-uniform traffic case, it is important to remember that, as mentioned previously, the majority of $s-d$ pairs in the non-uniform traffic matrix have a low traffic demand. A small percentage of $s$ - $d$ pairs have the higher traffic demands.

With this in mind, it is clear to see why, in all cases, fewer wavelengths are needed to achieve $100 \%$ throughput when using a non-uniform traffic matrix. As the majority of $s$ - $d$ pairs have a low traffic demand, reuse of wavelengths and virtual connections can occur more easily. More $s-d$ pairs can be routed over the same virtual connection when their traffic demands are low. This ultimately results in a requirement for fewer wavelengths, and lower network costs.

This reasoning can also be used to explain why throughput is higher when using a non-uniform traffic matrix. With lower average traffic demands, more $s-d$ pairs can be routed over fewer wavelengths.

Average delay is lower for all routing policies when a uniform traffic matrix is used. This again is due to the fact that in a non-uniform traffic matrix, the majority of $s-d$ pairs have low traffic demands. As each of these $s-d$ pairs is routed, there is a higher probability that the simulator will find an existing route with enough remaining capacity to handle the traffic. There is also an increased probability that this existing route will take a longer route than a newly created virtual link. This results in higher propagation 
delays, and ultimately, higher average delays when a non-uniform traffic matrix is used. However, the delay packets experience is still restrained by the maximum allowable delay as discussed in Section 4.2.

These results bode well as a non-uniform traffic matrix is a better representation of real-world traffic (a few $s-d$ pairs with a higher traffic demand) than a uniform traffic matrix.

\subsection{Comparison of Routing Policies}

Descriptions of the four routing policies were given in section 4.5. In this section, a comparison of the various routing schemes is discussed. Routing policy 1 will be compared with routing policy 2 , and routing policy 3 will be compared with routing policy 4 to determine which routing scheme of the pair is better. A comparison of the two best routing policies will then be conducted.

\subsubsection{Routing Policy 1 vs Routing Policy 2}

These two routing policies sort $s-d$ pairs solely on their traffic demand. Routing policy 1 routes $s-d$ pairs with the lowest traffic demand first, while routing policy 2 routes $s-d$ pairs with the higher traffic demand first. There is not a significant difference between the two routing policies when considering average packet delay (see Figure 5-8 and Figure 5-9 for example) to make any conclusions; as such, throughput will be used for comparison.

When looking at throughput (see Figure 5-4 and Figure 5-5 for example), routing policy 2 consistently produces higher average throughput values than routing policy 1 . It 
is also important to note that routing policy 2 uses less wavelengths to route $100 \%$ of the traffic. It can be argued that the throughput is higher only because the higher traffic values are routed first, increasing the total traffic routed and in turn, increasing the average throughput for any given $|W|$. However, the fact that fewer wavelengths are required for routing policy 2 indicates that this is a better method of routing $s-d$ pairs. The method of routing the higher traffic demands first allows better utilization of the wavelengths. In routing policy 1 , once the $s$ - $d$ pairs with the higher traffic demands are being routed, there is rarely enough capacity left on the virtual links to handle the traffic (since the prior $s$ - $d$ pairs with lower traffic demands have filled up the link). As such, additional wavelengths are required for only one or two virtual links. Routing policy 2 gets around this problem by routing the higher traffic demands first, allowing the higher wavelengths to be better utilized.

\subsubsection{Routing Policy 3 vs Routing Policy 4}

These two routing policies sort $s-d$ pairs solely on the number of hops between the two nodes. Routing policy 3 routes $s$ - $d$ pairs with the longest physical distance first, while routing policy 4 routes $s$ - $d$ pairs with the shortest physical distance first.

Routing policy 4 consistently produces results that have a higher throughput, and lower delay per $|\mathrm{W}|$ than routing policy 3 (see Figure 5-6, Figure 5-7, Figure 5-10 and Figure 5-11 for example). In routing policy 4 , all $s$ - $d$ pairs that are only one physical hop apart are routed first. This would produce a virtual topology that very closely resembles the physical topology. When $s-d$ pairs that are greater than one physical hop apart are 
routed, existing virtual links can be easily utilized as they are only one physical hop long. This allows for a greater probability for wavelength reuse, and a higher throughput value per $|W|$. Average delay also would be lower since the shortest distance between $s-d$ pairs can be exploited using existing one-hop virtual links.

Routing policy 3 routes the s-d pairs that are furthest apart first, making reuse of existing virtual links more difficult. Where reuse does occur, the path would be longer resulting in a higher average delay value.

\subsubsection{Routing Policy 2 vs Routing Policy 4}

The previous two sections compared the routing policies using the same sorting method. Routing policy 2 and routing policy 4 were shown to be the better choices. This section discusses which of these is the best overall choice.

Routing policy 2 consistently produces higher throughput values and higher average delay values per $|W|$. Higher delay values are caused because routing policy 2 does not necessarily use the shortest path. Routing policy 4, as mentioned previously, has very short virtual links allowing an increased probability that traffic will be routed on the shortest path.

This is not necessarily a negative point since all connections are still bounded by a maximum delay value. Therefore, sorting $s-d$ pairs according to their traffic demand is a better method of routing traffic than using physical distance as the sorting criteria. Overall, routing policy 2 is the better choice of routing $s-d$ pairs. 


\section{CHAPTER 6-CONCLUSIONS AND FUTURE RESEARCH}

\subsection{Conclusions}

This thesis introduces a new optical switch architecture, the half-clear wavelength convertible switch architecture. It compared the performance of this new architecture with the four optical switch architectures previously discussed in the literature. The halfclear wavelength convertible switch appears to be a good compromise between cost and performance.

The use of wavelength converters within the switch architectures was compared by considering the individual components characteristics in Chapter 3. It was shown that the dedicated wavelength convertible switch was more flexible, but was cost prohibitive due to the number of wavelength converters it employed. The electronic wavelength convertible switch architecture was cheap, but did not have the capability of providing a clear wavelength conversion, thus limiting performance. The share-per-node, share-perlink and half-clear wavelength convertible switch architectures were shown to be better choices with less cost than a dedicated wavelength convertible switch while retaining the capability of providing optical conversion. With only having the capability of optical conversion on half of the wavelengths in the network, the half-clear wavelength convertible switch was shown to be a very good compromise between cost and performance. 
To further investigate the performance of the half-clear wavelength convertible switch, it was tested through simulation against the no conversion case, and full conversion case (similar to the dedicated wavelength convertible switch). It was shown in Chapter 5 that the half-clear wavelength convertible switch architecture had similar performance characteristics as the dedicated wavelength convertible switch architecture. Both switches performed better than a network where conversion was not available.

The half-clear wavelength convertible switch was shown to have identical performance to the dedicated wavelength convertible switch architecture, but with costs similar to opto-electronic techniques. Therefore, the half-clear wavelength convertible switch architecture is a valid alternative to other switch architectures employing wavelength conversion. Its architecture is flexible enough to be configured independently at each node to allow specific wavelengths access to clear conversion. This should allow the network to route any traffic requirements presented.

By comparing uniform traffic against non-uniform traffic, it was shown that a non-uniform traffic matrix produced better results under the simulation conditions than a uniform traffic matrix when applied to the half-clear wavelength convertible switch architecture. As a non-uniform traffic matrix is more representative of real life traffic requirements (with a small number of nodes having higher traffic demands), this showed that these switches perform well under these conditions.

In comparing routing policies, it was shown that ignoring traffic considerations when routing $s-d$ pairs did not produce the best results. Having a routing policy that routes s-d pairs with a high traffic demand as the priority seems to be the best option. 


\subsection{Future Research}

As an extension to this work, the following list of open problems is suggested as potential future research areas:

- This work concentrated on the static-static routing and wavelength assignment approach. The work can be extended to consider the other approaches to routing and wavelength assignment such as the static-dynamic RWA, adaptive RWA, or dynamic RWA as described in Section 2.4. One approach to this problem would be to find the optimum virtual topology for every kind of traffic pattern. Then, find all possible combinations of virtual topologies where each virtual topology represents a different traffic pattern. Upon finding the combination of virtual topologies with the minimum network cost (minimum $|W|$ ) would be the solution for all traffic patterns. A starting point for this type of work can be found in [2] or [18].

- This work did not consider network survivability. In order for a network to be reliable, the failure of any single node of fibre link must not bring down the entire network. As such, virtual topologies should be designed with this in mind. Checks should be made on each node and link to determine if a failure would break the network into two or more separate pieces. One solution to this problem would be to provide alternate paths for virtual connections should a failure be detected. The more paths that are available, the more reliable the network would be, at the cost of increased price. More information on this problem can be found in [13] and [31]. 
- This work did not consider bifurcated traffic such as in an IP network. As an extension to this work, an investigation into the use of bifurcated traffic can be considered. This would require modification to the WCONSIM algorithm within the RWA module to allow for multiple paths between $s-d$ pairs. Some that may help in solving the bifurcated traffic problem include [32] and [33].

- This work considered the multiple-fibre node architecture only to take advantage of left over ports on the optical space switches. An investigation into the single fibre node architecture can also be done to determine the differences under the same conditions.

- The forecast of traffic patterns is crucial in developing a good network design. Performance difficulties are inevitable if the forecast is wrong or the average traffic values do not accurately reflect traffic behavior (bursty traffic). Queue size within the network nodes will also have an impact on performance; large queue sizes will increase the delay experienced by each packet, small queue sizes will result in increased probability of packet loss. To minimize the effects of dynamic traffic on the virtual topology, a sensitivity analysis should be conducted. This would involve modifying the maximum link utilization variable $\rho_{\max }$. Some references that would be useful in solving this problem include [17] and [34].

- This work did not research the optimum number of wavelength converters required in the architectures employing converter banks. Future research could concentrate on this problem, investigating the effects on performance that reducing the number of converters would cause. 


\section{LIST OF REFERENCES}

[1] Mukherjee, B., "WDM-Based Local Lightwave Networks Part 1: Single-Hop Systems," IEEE Network, Vol. 6, No. 3, May 1992, p. 12.

[2] Mukherjee, B., Optical Communication Networks, McGraw-Hill, 1997.

[3] Lee, K-C and Li, V.O.K., "A Wavelength-Convertible Optical Network," IEEE Journal of Lightwave Technology, Vol. 11, No. 5/6, May/June 1993, pp. 962 970.

[4] Kovacevic, M. and Acampora, A.S., "Electronic Wavelength Translation in Optical Networks," IEEE Journal of Lightwave Technology, Vol. 14, No. 6, Jun 96, pp. 1161-1169.

[5] Ramamurthy, B. and Mukherjee, B., "Wavelength Conversion in WDM Networking," IEEE Journal on Selected Areas in Communications, Vol. 16, No. 7, Sep 1998, pp. $1061-1073$.

[6] Wiesenfeld, J.M., "Wavelength conversion techniques," Proceedings, Optical Fiber Communication (OFC '96), San Jose, CA, vol. Tutorial TuP 1, pp. 71-72, 1996.

[7] Yoo, S.J.B., "Wavelength conversion technologies for WDM network applications," IEEE/OSA Journal of Lightwave Technology, vol. 14, pp. 955-966, June 1996.

[8] Danielsen, S.L., Hansen, P.B., Stubkjaer, K.E., "Wavelength Conversion in Optical Packet Switching," IEEE Journal of Lightwave Technology, Vol. 16, No. 12, Dec 98, pp. 2095-2108.

[9] Barry, R.A. and Humblet, P.A., "Models of Blocking Probability in All-Optical Networks with and without Wavelength Changers," IEEE Journal on Selected Areas in Communication, Vol. 14, No. 5, June 1996, pp. 858-867

[10] Subramaniam, S., Azizoglu, M., Somani, A.K., "All-Optical Networks with Sparse Wavelength Conversion," IEEE/ACM Transactions on Networking, Vol. 4, No. 4, Aug. 96, pp. 544-557.

[11] Mukherjee, B., Banerjee, D., Ramamurthy, S., Mukherjee, A., "Some Principals for Designing a Wide-Area WDM Optical Network," IEEE/ACM Transactions on Networking, Vol. 4, No. 5, Oct. 96, pp. 684-695. 
[12] Ramaswami, R., and Sivarajan, K., "Routing and Wavelength Assignment in AllOptical Networks," IEEE/ACM Transactions on Networking, Vol. 3, No. 5, October 1995, pp. 489-500.

[13] Ramaswami, R., and Sivarajan, K., Optical Networks: A Practical Perspective, Morgan Kaufmann, San Francisco, 1998.

[14] Naas, N.A., "Towards Optimum Design of Static Wavelength-Routing Optical Wide-Area Packet-Switched Networks," M.Sc.(Eng.) Thesis, Queen's University, Ontario, 1999.

[15] Chlamtac, I., Ganz, A., and Karmi, G., "Lightnets: Topologies for high speed optical networks," IEEE/OSA Journal of Lightwave Technology, Vol. 11, May/June 93, pp. 951-961.

[16] Ravikumar, C.P., Rai, T., and Verma, V., "Kautz graphs as attractive logical topologies in multihop lightwave networks," Computer Communications, Vol. 20., 1997, pp. 1259-1270.

[17] Zhang, Z., and Acampora, A., "A Heuristic Wavelength Assignment Algorithm for Multihop WDM Networks with Wavelength Routing and Wavelength Reuse," IEEE/ACM Transactions on Networking, Vol. 3, No. 3, Jun 95, pp. 281-288.

[18] Labourdette, J., Hart, G., and Acampora, A., "Branch-Exchange Sequences for Reconfiguration of Lightwave Networks," IEEE Transactions on Communications, Vol. 42, No. 10, Oct 94, pp. 2822-2832.

[19] Ramaswami, R., and Segall, A., "Distributed Network Control for Wavelength Routed Optical Networks," Proceedings IEEE Infocom 96, San Francisco, California, 1996, pp. 138-147.

[20] JDS Uniphase web site, http://www.jdsunph.com/, 1999.

[21] JDS Uniphase, "Product bulletin - SW Series Fibre Optic Switches", Feb. 1999.

[22] Astarté Fibre Networks Inc. web site, http://www.starswitch.com, 1999.

[23] Astarté Fibre Networks, "Product information - Star*Switch 7200," 1999.

[24] ADC/AOFR web site, http://www.adc.com/, 1999.

[25] ADC/AOFR, “Dense Wavelength Division Mux/DeMux," Aug. 98. 
[26] Alcatel web site, http://www.alcatel.com/, 1999.

[27] Alcatel, “1901 ICM Product Information,” 1999.

[28] Schwartz, M., Telecommunication Networks: Protocols, Modeling and Analysis, Addison Wesley, Reading, MA, 1988.

[29] Law, A.M., and Kelton, W.D., Simulation Modeling and Analysis, $2^{\text {nd }}$ Edition, McGraw Hill, New York, 1991.

[30] Naas, N. and Mouftah, H.T., "Design of Static Wavelength-Routing in Wide-Area Optical Packet-Switching Networks," Proceedings IEEE BSS'99, Kingston, Ontario, Canada, June 1999, pp. 93-97.

[31] Garnot, M., Sotom, M., and Masetti, F., "Routing Strategies for Optical Paths in WDM Networks," Proceedings IEE ICC '97, Montreal, Canada, June 1997, pp. 422-426.

[32] Lowe and Hunter, "Performance of Dynamic Path Optical Networks," IEE Proceedings on Optoelectronics, Vol. 144, No. 4, August 1997, pp. 235-239.

[33] Aggarwal, A., et. al., "Efficient Routing in Optical Networks," Journal of the Association for Computing Machinery, Vol. 43, No. 6, November 1996, pp. 973 1001 .

[34] Stern, T.E. et. al., "Linear Lightwave Networks: Performance Issues," IEEE/OSA Journal of Lightwave Technology, Vol. 11, No. 5/6, May/June 1993, pp. 937-950. 


\section{BIBLIOGRAPHY}

Azizoglu, M., Barry, R., and Mokhtar, A., "Impact of Tuning Delay on the Performance of Bandwidth-Limited Optical Broadcast Networks with Uniform Traffic," IEEE Journal on Selected Areas in Communication, Vol. 14, No. 5, June 1996, pp. 935 944.

Banerjee, D., and Mukherjee, B., "A Practical Approach for Routing and Wavelength Assignment in Large Wavelength-Routed Optical Networks," IEEE Journal on Selected Areas in Communication, Vol. 14, No. 5, June 1996, pp. 903-908.

Banerjee, S., and Mukherjee, B., "FairNet: A WDM-based Multiple Channel Lightwave Network with Adaptive and Fair Scheduling Policy," IEEE Journal of Lightwave Technology, Vol. 11, No. 5/6, May/June 1993, pp. $1104-1111$.

Banerjee, S., Yoo, J., and Chen, C., "Design of Wavelength-Routed Optical Networks for Packet Switched Traffic," IEEE Journal of Lightwave Technology, Vol. 15, No 9, Sep 1997, pp. $1636-1646$.

Barry, L., Guignard, P., et. al., "A High-Speed Optical Star Network Using TDMA and All-Optical Demultiplexing Techniques," IEEE Journal on Selected Areas in Communication, Vol. 14, No. 5, June 1996, pp. 1030-1038.

Barry, R., Chan, V., et. al., "All-Optical Network Consortium - Ultrafast TDM Networks," IEEE Journal on Selected Areas in Communication, Vol. 14, No. 5 , June 1996, pp. $999-1013$.

Birman, A., "Computing Approximate Blocking Probabilities for a Class of All-Optical Networks," IEEE Journal on Selected Areas in Communication, Vol. 14, No. 5, June 1996, pp. 852-857.

Blumenthal, D., Prucnal P., and Sauer, J., "Photonic Packet Switches: Architectures and Experimental Implementations," Proceedings of the IEEE, Vol. 82, No. 11 , November 1994, pp. $1650-1667$.

Borella, A., Cancellieri, G., and Chiraluce, F., Wavelength Division Multiple Access Optical Networks, Artech House, 1998. 
Borella, M., and Mukherjee, B., " Efficient Scheduling of Non-uniform Packet Traffic in a WDM/TDM Local Lightwave Network with Arbitrary Transceiver Tuning Latencies," IEEE Journal on Selected Areas in Communication, Vol. 14, No. 5, June 1996, pp. $923-934$.

Brackett, C., "Dense Wavelength Division Multiplexing Networks: Principles and Applications," IEEE Journal on Selected Areas in Communication, Vol. 8, No. 6, August 1990, pp. $948-963$.

Dutta, R., and Rouskas, G.N., "A Survey of Virtual Topology Design Algorithms for Wavelength Routed Optical Networks," from the Internet.

Hinton, H., "Photonic Switching Using Directional Couplers," IEEE Communications Magazine, Vol. 25, No. 5, May 1987, pp. 16-25.

Hluchyj, M.G., and Karol, M.J., "ShuffleNet: An Application of Generalized Perfect Shuffles to Multihop Lightwave Networks," IEEE Journal of Lightwave Technology, Vol. 9, No. 10, Oct 1991, pp. 1386-1396.

Hui, J.Y., Switching and Traffic Theory for Integrated Broadband Networks, Kluwer Academic Publishers, 1990.

Jajszczyk, A., and Mouftah, H., "Photonic Fast Packet Switching," IEEE Communications Magazine, February 1993, pp. 58 - 65.

Jajszczyk, A., and Mouftah, H., "Tree-Type Photonic Switching Networks," IEEE Network, January/February 1995, pp. $10-15$.

Kleinberg, J., and Kumar, A., "Wavelength Conversion in Optical Networks," Proceedings of the Annual ACM SIAM Symposium on Discrete Algorithms, Philadelphia, U.S.A., 1999, pp. 566-575.

Kovacevic, M., and Acampora, A., "Benefits of Wavelength Translation in All-Optical Clear-Channel Networks," IEEE Journal on Selected Areas in Communication, Vol. 14, No. 5, June 1996, pp. 868-880.

Labourdette, J-F.P., and Acampora, A.S., "Logically Rearrangeable Multihop Lightwave Networks," IEEE Transactions on Communications, Vol. 39, No 8, Aug 1991, pp. 1223-1230.

Ladd, S.R., C++ Templates and Tools, M\&T Books, 1995. 
Masetti, F., Benoit, J., et. al., "High Speed, high Capacity ATM Optical Switches for Future Telecommunication Transport Networks," IEEE Journal on Selected Areas in Communication, Vol. 14, No. 5, June 1996, pp. $979-998$.

Mukherjee, B., "WDM-Based Local Lightwave Networks Part 2: Multi-Hop Systems," IEEE Network, Vol. 6, No. 4, July 1992, pp. 20-32.

Ramaswami, R., and Sivarajan, K., "Design of Logical Topologies for WavelengthRouted Optical Networks," IEEE Journal on Selected Areas in Communication, Vol. 14, No. 5, June 1996, pp. 840-851.

Sabella, R., Iannone, E., and Pagano, E., "Optical Transport Networks Employing AllOptical Wavelength Conversion: Limits and Features," IEEE Journal on Selected Areas in Communication, Vol. 14, No. 5, June 1996, pp. 968-978.

Seo, S., Bergman, K., and Prucnal, P., "Transport Optical Networks with Time Division Multiplexing," IEEE Journal on Selected Areas in Communication, Vol. 14, No. 5 , June 1996, pp. $1039-1051$.

Sivarajan, K., and Ramaswami, R., "Lightwave Networks Based on de Bruijn Graphs," IEEE/ACM Transactions on Networking," Vol. 2, No. 1, Feb 1994, pp. 70-79.

Spanke, R., "Architectures for Guided-Wave Optical Space Switching Systems," IEEE Communications Magazine, Vol. 25, No. 5, May 1987, pp. 42-48.

Stern, T.E. and Bala, K., Multiwavelength Optical Networks: A Layered Approach, Addison-Wesley, 1999.

Tada, Y., et. al., "OA\&M Framework for Multi-wavelength Photonic Transport Networks," IEEE Journal on Selected Areas in Communication, Vol. 14, No. 5, June 1996, pp. 914-922.

Tanenbaum, A.S., Computer Networks, $3^{\text {rd }}$ Edition, Prentice Hall PTR, 1996.

Tridandapani, S., and Mukherjee, B., "Channel Sharing in Multi-Hop WDM Lightwave Networks: Realization and Performance of Multicast Traffic," IEEE Journal on Selected Areas in Communication, Vol. 15, No. 5, April 1997, pp. 488-500. 


\section{VITA}

Name:

Place and Year of birth:

Education:

Experience:
Nicholas Patrick Torrington-Smith

Portsmouth, England, 1971

Mount Allison University, 1989-1990

Royal Roads Military College, 1990-1993

awarded B Sc (Computer Science / Math) 1993

Royal Military College, 1999-2000

Post Graduate Training Program (Comp Eng)

Joined the Canadian Forces in 1990

Graduated from the Canadian Forces School of

Communications and Electronics in 1994

Employed within the Wing Telecommunications and Information Services Squadron at 14 Wing Greenwood, 1994 - 1999. 